\title{
Índice de classes de coincidências em superfícies
}

\section{Daniel Vendrúscolo}

\author{
TESE APRESENTADA \\ AO \\ INSTITUTO DE MATEMÁTICA E ESTATÍSTICA \\ DA \\ UNIVERSIDADE DE SÃO PAULO \\ PARA \\ OBTENÇÃO DO GRAU DE DOUTOR \\ EM \\ MATEMÁTICA
}

\begin{abstract}
Área de Concentração: Topologia Algébrica Orientador: Prof. Dr. Daciberg Lima Gonçalves
\end{abstract}

Durante a elaboração deste trabalho o autor recebeu apoio financeiro da FAPESP, processo 97/13623-1.

-São Paulo, 26 de Abril de 2002- 


\title{
Índice de classes de coincidências em superfícies
}

\author{
Este exemplar corresponde à redação \\ final da tese devidamente corrigida e \\ defendida por Daniel Vendrúscolo \\ e aprovada pela comissão julgadora.
}

São Paulo, 26 de Abril de 2002.

Banca examinadora:

- Prof. Dr. Daciberg Lima Gonçalves (Orientador) - IME - USP

- Profa. Dra. Lucília Daruiz Borsari - IME - USP

- Prof. Dr. Carlos Biasi - ICMC - USP.

- Prof. Dr. Pedro Luiz Queiroz Perger - UFSCar

- Prof. Dr. Derek Douglas Jack Hacon - PUC - RJ 


\section{Agradecimentos}

Ao Prof. Daciberg pelo seu interesse e apoio, além de sua orientação indispensável e precisa.

Ao grupo de topolologia algébrica do IME-USP: Daciberg, Lucília, Fernanda, Pedro, Claudemir e Toninho; pela melhor das convivências.

Aos grandes e sinceros amigos que fiz no IME; na esperança que essa amizade realmente nunca se perca.

Ao Bologna e ao Xirim, pois não há como não agradecer.

À Paula, pelo interesse e pela compreensão.

À Liane; que essa ajuda nunca me falte. 


\title{
Resumo
}

Nesse trabalho demonstramos alguns resultados sobre limitações para o índice de uma classe de Nielsen de coincidência de aplicações entre superfícies. Usando a definição de índice para o caso não orientável mostramos que quando o domínio tem genus maior do que 1, é diferente do contra-domínio e nenhuma das aplicações é homotópica a um revestimento o índice para as classes de coincidência não é limitado. Além disso provamos que quando o domínio é o toro ou a garrafa de Klein, tais índices são limitados.

\begin{abstract}
In this work we present several results about bounds of coincidence indeces of Nielsen coincidence classes of maps between surfaces. We consider the definition of coincidence index in the non orientable case and we prove that when the domain does not have genus 1 , is different from the counter-domain and both maps are not homotopic to a covering map, then the coincidence class index is not bounded. We also prove that when the domain is the torus or the Klein bottle these indices are bounded.
\end{abstract}




\section{Índice}

Agradecimentos $\quad$ ii

Resumo iii

$\begin{array}{lll}\text { Abstract } & \text { iii }\end{array}$

Introdução vi

1 Homologia com Coeficientes Locais 1

1.1 Feixe de Grupos . . . . . . . . . . . . . . . . . . . 1

1.2 Homologia com Coeficientes Locais . . . . . . . . . . . . . . 6

1.3 Homologia Relativa . . . . . . . . . . . . . . . . . . 8

1.4 Homologia de um Ponto de Vista Axiomático . . . . . . . . . . . . 9

1.4.1 Homotopias em $\mathbb{L} \ldots \ldots$. . . . . . . . . . . . . . . 10

1.4 .2 "Axiomas" ..................... . . 10

1.5 Sequência de Mayer-Vietoris . . . . . . . . . . . . . . . 12

1.6 Cohomologia com Coeficientes Locais . . . . . . . . . . . . . . 18

1.6.1 Cohomologia de um Ponto de Vista Axiomático . . . . . . 19

1.7 Alguns Exemplos . . . . . . . . . . . . . . . . . 20

1.7.1 O Plano Projetivo . . . . . . . . . . . . . . . 21 
1.7 .2 A Garrafa de Klein . . . . . . . . . . . . . . . . . 24

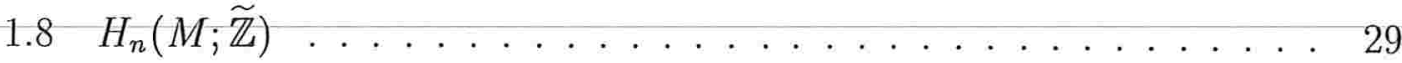

2 Preliminares $\quad 34$

2.1 Grau de Aplicações . . . . . . . . . . . . . . . . . . . . . . 34

2.2 Aplicações Fiéis à Orientação . . . . . . . . . . . . . . . 36

2.3 Classes de Coincidências . . . . . . . . . . . . . . . 36

2.4 Índice e Número de Lefschtez . . . . . . . . . . . . . . . . . . . . . 38

2.5 Número de Lefschetz em Superfícies . . . . . . . . . . . . . . . . . 40

3 O caso orientável $\quad 44$

3.1 Aplicações do Toro . . . . . . . . . . . . . . . . . . . 46

3.1.1 Um pouco da geometria de $S_{g} \ldots \ldots \ldots$. . . . . 47

3.1 .2 Forma padrão de $f: S_{1} \mapsto S_{g} \ldots \ldots \ldots . \ldots 48$

4 O caso não orientável $\quad \mathbf{5 4}$

4.1 Aplicações de $K_{h} \mapsto K_{g} \quad \ldots \ldots \ldots \ldots$. . . . . . . . . . 54

4.1 .1 A garrafa de Klein . . . . . . . . . . . . . . . . . 54

$4.1 .2 \quad h \geq 1 \ldots \ldots \ldots \ldots \ldots$

4.2 Aplicações de $K_{h} \mapsto P_{g} \ldots \ldots \ldots \ldots$. . . . . . . . 71

4.3 Outros Casos . . . . . . . . . . . . . . . . . 78

4.4 Alguns Resultados com Limitação . . . . . . . . . . . . . . . . 78

4.4 Recobrimentos .................. 78

$4.4 .2 \quad B_{K}(g, g, 0,1)$ e $B_{P}(g, g, 0,1) \ldots \ldots \ldots$

$\begin{array}{ll}\text { Referências Bibliogáficas } & 81\end{array}$ 


\section{Introdução}

O objetivo desse trabalho é apresentar alguns resultados sobre a limitação ou não dos índices de classes de Nielsen de coincidências para aplicações entre superfícies orientáveis ou não. O aparecimento dessa questão ocorreu, inicialmente, no contexto de ponto fixo.

Os primeiros resultados sobre limitação para índice de pontos fixos apareceram no estudo de homeomorfismos de superfícies (ver [Simon], [Pelikan \& Slaminka] e [Jiang \& Guo] por exemplo). Em [Jiang 2], [Kelly 1] e [Kelly 2] são apresentados alguns resultados sobre limitações para índices de classes de Nielsen de ponto fixo para auto-aplicações de superfícies com ou sem bordo.

Nesse contexto desponta naturalmente a questão sobre a validade de tais resultados para coincidências de um par de aplicações. Em [Brooks] ou [BBPT] podemos encontrar resultados sobre a limitação para índices de classes de coincidência para pares de aplicações no toro. Mais recentemente, em [Gonçalves \& Jiang], encontramos resultados negativos sobre limitações para o índice de uma classe de coincidência em um grande espectro de casos para aplicações entre superfícies orientáveis, além de alguns resultados positivos em situações específicas.

Para superfícies não orientáveis a questão da limitação para o índice de classes de coincidência é precedida pela questão da definição de tal índice. Em [Dold] encontramos a definição de índice de ponto fixo para ENRs e sabe-se que tal definição não pode ser estendida para coincidências. Em [Gonçalves] obtemos uma versão, "via" obstrução, de um índice para coincidências não só para variedades não orientáveis mas para uma classe bem geral de aplicações de um complexo em uma variedade. Simultaneamente em [Dobreńko \& Jezierski] desenvolveu-se uma definição "geométrica" de índice para classes de Nielsen de coincidências para aplicações entre variedades diferenciáveis. Na sequência, em [Jezierski], é apresentada uma versão de tal índice para variedades topológicas e em [Gonçalves \& Jezierski] 
encontramos uma "fusão" das duas vertentes quando uma das aplicações é fiel à orientaçã $o^{1}$.

Para o estudo específico de superfícies, [Gonçalves \& Oliveira] nos fornece fórmulas para o cálculo do número de Lefschetz quando uma das aplicações é fiel à orientação.

No início desse trabalho (Capítulo 1) apresentamos de maneira sucinta a teoria de Homologia com Coeficientes Locais que é a principal ferramenta para a construção de um índice de coincidência e de um número de Lefschetz no caso não orientável. No Capítulo 2 apresentamos alguns resultados de [Gonçalves \& Jezierski] e [Gonçalves \& Oliveira] que serão úteis posteriormente além da definição de grau de uma aplicação entre variedades não orientáveis ([Olum 2]).

No Capítulo 3 tratamos de algumas situações entre superfícies orientáveis que ainda não estavam completas na literatura e finalmente no Capítulo 4 apresentamos os resultados obtidos para aplicações entre superfícies não necessariamente orientáveis com a restrição de uma das aplicações ser fiel à orientação. Cabe ressaltar que os resultados obtidos são bastante semelhantes aos existentes para o caso orientável.

\footnotetext{
${ }^{1}$ Definição na página 36
} 


\section{Homologia com Coeficientes Locais}

Nesse capítulo apresentaremos a teoria básica de homologia e cohomologia com coeficientes locais, tal teoria será usada posteriormente quando estudarmos aplicações entre superfícies não orientáveis. As referências para esse tema são [Whitehead], [Baues], [Olum 1], [Olum 2], [Steenrood 1] e [Steenrood 2].

\subsection{Feixe de Grupos}

Sendo $B$ um espaço topológico e denotando-se por $\Pi_{1}(B)$ a categoria cujos objetos são os pontos de $B$ e os morfismos são dados por elementos dos conjuntos $\pi_{1}\left(B ; b_{1}, b_{2}\right)$ (classe de homotopia das curvas em $B$ de $b_{1}$ até $b_{2}$ ), podemos definir: Definição 1.1. Um feixe de grupos (abelianos) é um funtor contravariante $G$ de $\Pi_{1}(B)$ em $\mathfrak{G}$ a categoria dos grupos, (A), a categoria dos grupos abelianos).

Dessa forma para cada $b \in B, G(b)$ é um grupo (abeliano) e para cada classe $\xi \in \pi_{1}\left(B ; b_{1}, b_{2}\right), G(\xi): G\left(b_{2}\right) \rightarrow G\left(b_{1}\right)$ é um homomorfismo de grupos (abelianos) satisfazendo:

1. se $\xi \in \pi_{1}(B ; b)=\pi_{1}(B ; b, b)$ é a identidade então

$$
G(\xi): G(b) \rightarrow G(b)
$$

é a identidade.

2. se $\xi \in \pi_{1}\left(B ; b_{1}, b_{2}\right)$ e $\eta \in \pi_{1}\left(B ; b_{2}, b_{3}\right)$, então

$$
G(\xi \eta)=G(\xi) \circ G(\eta): G\left(b_{3}\right) \rightarrow G\left(b_{1}\right) .
$$


Segue daí que:

3. se $\xi \in \pi_{1}\left(B ; b_{1}, b_{2}\right)$, então

$$
G\left(\xi^{-1}\right)=G(\xi)^{-1}: G\left(b_{1}\right) \rightarrow G\left(b_{2}\right)
$$

$\operatorname{assim} G(\xi)$ é um isomorfismo.

Em outras palavras $G$ pode ser pensado como um funtor que para qualquer ponto de $B$ associa um grupo fixado (mas vistos de maneira distinta) e para cada classe de homotopia em $\pi_{1}\left(B ; b_{1}, b_{2}\right)$ associa um automorfismo desse grupo (mas devemos ressaltar que $G\left(b_{1}\right)$ e $G\left(b_{2}\right)$ estão sendo vistos de maneira distinta).

No caso de $G$ ser um funtor com imagem nos grupos abelianos dizemos que $G$ é um Sistema de Coeficientes Locais.

Definição 1.2. Se $G$ e $H$ são feixes de grupos, um homomorfismo de feixes de grupos $\Phi: G \rightarrow H$ é uma transfomação natural de funtores, ou seja, uma aplicação que para cada $b \in B$ associa $\phi(b): G(b) \rightarrow H(b)$ um homomorfismo que torna o diagrama

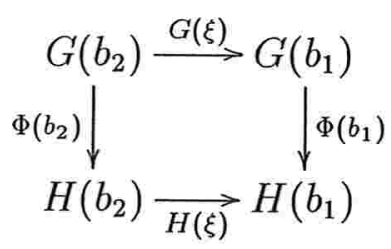

comutativo para qualquer $\xi \in \pi_{1}\left(B ; b_{1}, b_{2}\right)$.

Definição 1.3. $\Phi$ é um isomorfismo de feixes de grupos se e somente se cada $\Phi(b)$ for um isomorfismo.

Observamos que se $G$ é um feixe de grupos em $B$ então $\pi_{1}\left(B, b_{0}\right)$ age em $G\left(b_{0}\right)$. Além disso se $\pi_{1}\left(B, b_{0}\right)$ age em um grupo $G_{0}$ e se $B$ é conexo por caminhos, então existe um feixe $G$ em $B$ (único a menos de isomorfismos) tal que $G\left(b_{0}\right)=G_{0}$ e $G$ induz a mesma ação em $G\left(b_{0}\right)$. Mais especificamente temos os seguintes resultados:

Teorema 1.4. Se $B$ é conexo por caminhos, $G$ e $G^{\prime}$ feixes em $B$ e se $\phi: G\left(b_{0}\right) \rightarrow$ $G^{\prime}\left(b_{0}\right)$ é um isomorfismo que respeita a ação de $\pi_{1}\left(B, b_{0}\right)$. Então existe um único isomorfismo $\Phi: G \rightarrow G^{\prime}$ tal que $\Phi\left(b_{0}\right)=\phi$.

Demonstração: Para cada $b \in B$ escolhemos um elemento $\xi(b) \in \pi_{1}\left(B ; b_{0}, b\right)$; assumimos que $\xi\left(b_{0}\right)=1$ (a identidade em $\pi_{1}\left(B, b_{0}\right)$ ). 
Definimos $\Phi(b): G(b) \rightarrow G^{\prime}(b)$ por:

$$
\Phi(b)=G^{\prime}(\xi(b))^{-1} \circ \phi \circ G(\xi(b))
$$

e assim, se $\eta \in \pi_{1}\left(B ; b_{1}, b_{2}\right)$ temos

$$
\Phi\left(b_{1}\right) \circ G(\eta)=G^{\prime}\left(\xi\left(b_{1}\right)\right)^{-1} \circ \phi \circ G\left(\xi\left(b_{1}\right)\right) \circ G(\eta)=G^{\prime}\left(\xi\left(b_{1}\right)\right)^{-1} \circ \phi \circ G\left(\xi\left(b_{1}\right) \eta\right)
$$

e

$$
G^{\prime}(\eta) \circ \Phi\left(b_{2}\right)=G^{\prime}(\eta) \circ G^{\prime}\left(\xi\left(b_{2}\right)\right)^{-1} \circ \phi \circ G\left(\xi\left(b_{2}\right)\right)=G^{\prime}\left(\eta \xi\left(b_{2}\right)^{-1}\right) \circ \phi \circ G\left(\xi\left(b_{2}\right)\right) .
$$

Sendo $\alpha=\xi\left(b_{1}\right) \eta \xi\left(b_{2}\right)^{-1}$ temos que $\alpha \in \pi_{1}\left(B ; b_{0}\right)$ e como $\phi$ respeita a ação de $\pi_{1}\left(B, b_{0}\right)$ temos:

$$
\phi \circ G(\alpha)=G^{\prime}(\alpha) \circ \phi
$$

e assim:

$$
\begin{aligned}
\Phi\left(b_{1}\right) \circ G(\eta) & =G^{\prime}\left(\xi\left(b_{1}\right)\right)^{-1} \circ \phi \circ g\left(\alpha \xi\left(b_{2}\right)\right) \\
& =G^{\prime}\left(\xi\left(b_{1}\right)\right)^{-1} \circ \phi \circ G(\alpha) \circ G\left(\xi\left(b_{2}\right)\right) \\
& =G^{\prime}\left(\xi\left(b_{1}\right)\right)^{-1} \circ G^{\prime}(\alpha) \circ \phi \circ G\left(\xi\left(b_{2}\right)\right) \\
& =G^{\prime}\left(\xi\left(b_{1}\right)^{-1} \alpha\right) \circ \phi \circ G\left(\xi\left(b_{2}\right)\right) \\
& =G^{\prime}\left(\eta \xi\left(b_{2}\right)^{-1}\right) \circ \phi \circ G\left(\xi\left(b_{2}\right)\right) \\
& =G^{\prime}(\eta) \circ \Phi\left(b_{2}\right)
\end{aligned}
$$

Desse modo temos que $\Phi$ é uma transformação natural:

$$
\begin{aligned}
\Phi & =G^{\prime}\left(\xi\left(b_{0}\right)\right)^{-1} \circ \phi \circ G\left(\xi\left(b_{0}\right)\right) \\
& =G^{\prime}(1)^{-1} \circ \phi \circ G(1) \\
& =\phi .
\end{aligned}
$$

A unicidade da $\Phi$ segue do fato que se $b \in B$ e se $\xi$ é uma curva de $b_{0}$ até $b$ o diagrama

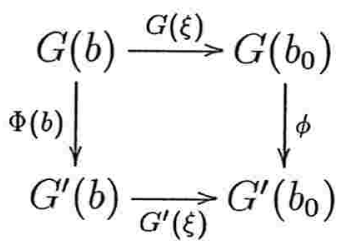

é comutativo e teremos:

$$
\Phi(b)=G^{\prime}(\xi)^{-1} \circ \phi \circ G(\xi)
$$


Teorema 1.5. Se $B$ é conexo por caminhos, $\mathbb{G}$ grupo no qual $\pi_{1}\left(B ; b_{0}\right)$ age. Então existe $G$, feixe de grupos em $B$ tal que $G\left(b_{0}\right)=\mathbb{G}$ e que induz a ação de $\pi_{1}\left(B ; b_{0}\right)$ em $G\left(b_{0}\right)$.

Demonstração: Novamente para cada $b \in B$ escolhemos um elemento $\xi(b) \in$ $\pi_{1}\left(B ; b_{0}, b\right), \operatorname{com} \xi\left(b_{0}\right)=1$.

Se $G(b)=\mathbb{G}$ para todo $b \in B$ e se $\eta \in \pi_{1}\left(B ; b_{1}, b_{2}\right)$ então $\xi\left(b_{1}\right) \eta \xi\left(b_{2}\right)^{-1} \in$ $\pi_{1}\left(B ; b_{0}\right)$, assim temos que:

$$
\xi\left(b_{1}\right) \eta \xi\left(b_{2}\right)^{-1}: \mathbb{G} \rightarrow \mathbb{G}
$$

pois $\pi_{1}\left(B ; b_{0}\right)$ age em $\mathbb{G}$.

Podemos então definir:

$$
G(\eta)=\xi\left(b_{1}\right) \eta \xi\left(b_{2}\right)^{-1}: G\left(b_{2}\right)=\mathbb{G} \rightarrow \mathbb{G}=G\left(b_{1}\right) .
$$

Nessas condições temos que:

1. Se $b_{1}=b_{2}$ e se $\eta=1 \in \pi_{1}\left(B ; b_{1}\right)$ então $\xi\left(b_{1}\right) \eta \xi\left(b_{1}\right)^{-1}=1$, ou seja $G(\eta)=1$.

2. Se $\delta \in \pi_{1}\left(B ; b_{2}, b_{3}\right)$ temos que

$$
\left(\xi\left(b_{1}\right) \eta \xi\left(b_{2}\right)^{-1}\right)\left(\xi\left(b_{2}\right) \delta \xi\left(b_{3}\right)^{-1}\right)=\xi\left(b_{1}\right) \eta \delta \xi\left(b_{3}\right)^{-1}
$$

ou seja $G(\eta \delta)=G(\eta) \circ G(\delta)$.

Portanto $G$ é um feixe de grupos em $B$ e se $b_{1}=b_{2}=b_{0}, \eta \in \pi_{1}\left(B ; b_{0}\right)$ temos que $\xi\left(b_{1}\right) \eta \xi\left(b_{2}\right)^{-1}=\eta$, o que mostra que $G$ induz a mesma ação em $G\left(b_{0}\right)$.

Vejamos agora alguns exemplos:

Exemplo 1.6. Um grupo $\mathbb{G}$ determina um feixe de grupos $G$ constante:

- $G(b)=\mathbb{G} \forall b \in B$.

- $G(\xi)=i d \quad \forall \xi \in \pi_{1}\left(B ; b_{1}, b_{2}\right)$.

Definição 1.7. Um feixe de grupos $G$ é dito simples se $G$ é isomorfo a um feixe constante.

É fácil ver que: 
Proposição 1.8. Um feixe de grupos $G$ é simples se, e somente se, $G(\xi)$ independe de $\xi$.

Exemplo 1.9. $G$ associa a cada ponto $b \in B$ o grupo de homotopia $\pi_{n}(B, b)$ e a cada elemento $\xi \in \pi_{1}(B ; b)$ a ação usual de $\pi_{1}(B ; b)$ em $\pi_{n}(B, b)$.

Esse feixe em $B$ é denotado $\Pi_{n}(B)$.

Exemplo 1.10. Se $B \subset A$ podemos, de maneira análoga ao exemplo 1.9, construir o feixe $\Pi_{n}(A, B)$ em $B$.

Exemplo 1.11. Se $p: X \rightarrow B$ é uma fibração então os grupos de homologia $H_{n}\left(F_{b}\right)$ das fibras com a ação usual de $\pi_{1}(B)$ em $H_{n}\left(F_{b}\right)$ formam um sistema de coeficientes locais em $B$.

Lembrando que se $\xi \in \pi_{1}(X), \operatorname{sinal}(\xi)=1$ ou -1 conforme $\xi$ preserve ou reverta a orientação local de $X$ temos:

Exemplo 1.12. Se $X$ é uma n-variedade conexa, tomando-se, para cada $x \in X$, $G(x)=H_{n}(X, X \backslash\{x\} ; \mathbb{G})$ e tomando-se a ação de $\pi_{1}(X)$ em $G(x)=H_{n}(X, X \backslash$ $\{x\} ; \mathbb{G})$ dada por:

$$
G(\xi)(g)=\left\{\begin{array}{lll}
g & \text { se } & \operatorname{sinal}(\xi)=1 \\
-g & \text { se } & \operatorname{sinal}(\xi)=-1
\end{array}\right.
$$

Então $G$ é o sistema de coeficentes locais dado pela orientação de $X$ com valores em $G$.

Observação 1.13. Quando $\mathbb{G} \approx \mathbb{Z}$ denotaremos $G$ por $\widetilde{\mathbb{Z}}$ e se $\mathbb{G} \approx \mathbb{Q}, G$ será denotado $\widetilde{\mathbb{Q}}$. Vale a pena observar que se $X$ é orientável o sistema de coeficentes locais dado pela orientação de $X$ coincide com o usual.

Observação 1.14. Se $\mathbb{G} \approx \mathbb{Z}_{2}$ o sistema de coeficientes locais induzido pela orientação corresponde ao feixe de grupos simples com valores em $\mathbb{Z}_{2}$.

Se $A$ e $B$ são espaços topológicos e $f: A \rightarrow B$ é uma aplicação a composição com $f$ induz um funtor $F: \Pi_{1}(A) \rightarrow \Pi_{1}(B)$. Categoricamente falando $\Pi_{1}$ é um funtor dos espaços topológicos nos grupóides e $\Pi_{1}(f)=F$. Se $G$ é um feixe de grupos em $B$ temos que $G \circ F$ é um feixe de grupos em $A$ (note que $G: \Pi_{1}(B) \rightarrow \mathfrak{G}$ e $\left.G \circ F: \Pi_{1}(A) \rightarrow \mathfrak{G}\right)$.

Definição 1.15. O feixe $f^{*}(G)=G \circ F$ sobre $A$ é chamado feixe induzido pela $f$.

Teorema 1.16. Se $f: A \rightarrow B$ e $G: B \rightarrow C$ são aplicações e $H$ é um feixe de grupos sobre $C$ então $(g \circ f)^{*}(H)=f^{*}\left(g^{*}(H)\right)$. 
Demonstração: Segue diretamente da definição.

Se $B$ é conexo por caminhos e admite recobrimento universal temos que se $b_{0} \in B$ e se $G$ é um feixe de grupos sobre $B$, tomando-se o subgrupo $K=\{\eta \in$ $\pi_{1}\left(B, b_{0}\right) \mid G(\eta)=i d$ em $\left.G\left(b_{0}\right)\right\} \subset \pi_{1}\left(B, b_{0}\right)$ e usando a teoria de espaços de recobrimento temos que existe $p: \tilde{B} \rightarrow B$, recobrimento de $B$ e $\tilde{b}_{0} \in p^{-1}\left(b_{0}\right)$ tais que $p_{*}\left(\pi_{1}\left(\tilde{B}, \tilde{b}_{0}\right)\right)=K$. É fácil ver que:

Proposição 1.17. Nas condições acima $p^{*}(G)$ é simples.

Também é fácil ver que:

Proposição 1.18. Seja $p: X \rightarrow B$ uma fibração com fibras n-simples. Se $f$ : $B^{\prime} \rightarrow B$ é uma aplicação e se $p^{\prime}: X^{\prime} \rightarrow B^{\prime}$ é a fibração induzida por $f$ então os sistemas de coeficientes locais $\pi_{n}(\mathfrak{F})$ e $\pi_{n}\left(\mathfrak{F}^{\prime}\right)$ estão relacionados pela expressão:

$$
\pi_{n}(\mathfrak{F})=f^{*} \pi_{n}\left(\mathfrak{F}^{\prime}\right)
$$

Vale lembrar que na Proposição acima temos $X^{\prime}=\left\{\left(b^{\prime}, x\right) \in B^{\prime} \times X \mid f\left(b^{\prime}\right)=\right.$ $p(x)\}$ e temos o seguinte diagrama comutativo:

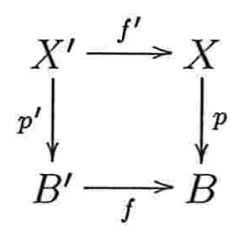

onde $f^{\prime}$ e $p^{\prime}$ são as projeções.

\subsection{Homologia com Coeficientes Locais}

Se $X$ é um espaço topológico e $G$ um sistema de coeficientes locais (um feixe de grupos abelianos ), definimos $C_{q}(X ; G)$ como o conjunto de todas as funções $c$ definidas nos $q$-simplexos singulares de $X$ que satisfazem:

1. Para cada $q$-simplexo singular $u: \Delta^{q} \rightarrow X, c(u)$ está definida e $c(u) \in$ $G\left(u\left(e_{0}\right)\right)$.

2. O conjunto dos $q$-simplexos singulares $u$ tais que $c(u) \neq 0$ é finito.

Os elementos de $C_{q}(X ; G)$ são as $q$-cadeias singulares com coeficientes em $G$. 
Definição 1.19. Uma cadeia singular c é dita elementar se $c(u) \neq 0$ para, no máximo, um simplexo singular $u$.

Notação: $c$ uma $q$-cadeia singular elementar será denotada $c=c(u) \cdot u$.

Cada $c \in C_{q}(X ; G)$ pode ser escrito como uma soma finita:

$$
c=\sum_{i=1}^{m} g_{i} u_{i}
$$

onde $u_{i}$ são simplexos singulares e $g_{i} \in G\left(u_{i}\left(e_{0}\right)\right)$.

Para definirmos um "operador bordo" em $C_{*}(X ; G)$ vamos introduzir alguma notação mais precisa.

Se $\mu: \Delta^{n} \rightarrow X$ é um simplexo singular temos que $\partial_{i} \mu$ é definido como a $i$-ésima face de $\mu$ (obtida "retirando-se o $i$-ésimo vértice de $\mu$ ), mais formalmente temos que se $e_{i}, \quad i=0, \ldots, n$, são os vértices de $\Delta^{n}$ tomando-se $d_{i}^{n}: \Delta^{n-1} \rightarrow \Delta^{n}$ uma aplicação afim satisfazendo:

$$
d_{i}^{n}\left(e_{j}\right)=\left\{\begin{array}{lll}
e_{j} & \text { se } & j<i \\
e_{j+1} & \text { se } & j \geq i
\end{array}\right.
$$

obtemos :

$$
\partial_{i} \mu=\mu \circ d_{i}^{n}
$$

Dessa forma observamos que:

$$
\partial_{i} \mu\left(e_{0}\right)=\left\{\begin{array}{lll}
\mu\left(e_{0}\right) & \text { se } & i>0 \\
\mu\left(e_{1}\right) & \text { se } & i=0
\end{array}\right.
$$

e uma definição para um "operador bordo" do tipo

$$
\partial(g \mu)=\sum_{i=0}^{n}(-1)^{i} g \partial_{i} \mu
$$

não é possível pois $g \in G\left(\mu\left(e_{0}\right)\right)$ mas, em geral, $g \notin G\left(\partial_{0} \mu\left(e_{0}\right)\right)$.

Uma formulação que se mostrará útil é obtida se notarmos que se $\gamma: I \rightarrow X$ é dada por $\gamma(t)=\mu\left((1-t) e_{1}+t e_{0}\right)$ (curva de $\mu\left(e_{1}\right)$ até $\left.\mu\left(e_{0}\right)\right)$ então a classe de homotopia de $\gamma$ induz um isomorfismo $G\left(\sigma_{\mu}\right): G\left(\mu\left(e_{0}\right)\right) \rightarrow G\left(\mu\left(e_{1}\right)\right)$ e assim podemos definir:

$$
\partial(g \mu)=G\left(\sigma_{\mu}\right)(g) \cdot \partial_{0} \mu+\sum_{i=1}^{n}(-1)^{i} g \partial_{i} \mu .
$$


$\partial \partial(g \mu)=0$ decorre do fato de $\sigma_{\partial_{i} \mu}=\sigma_{\mu}$ se $i>1$ e $\sigma_{\partial_{1} \mu}=\sigma_{\partial_{0} \mu} \circ \sigma_{\mu}$ (pois as curvas correspondentes são homotópicas).

Dessa forma $C_{*}(X ; G)$ é um complexo de cadeia e definimos:

$$
H_{q}(X ; G)=H_{q}\left(C_{*}(X ; G)\right)
$$

o Grupo de Homologia com Coeficientes Locais em $G$.

Teorema 1.20. Se o espaço $X$ é conexo por caminhos e $G$ é simples, então $H_{q}(X ; G)=H_{q}\left(X ; G_{0}\right)$ (grupo de homologia "tradicional") onde $G_{0}=G\left(x_{0}\right)$ para algum $x_{0} \in X$.

Demonstração: Decorre diretamente da definição de feixe simples.

Nesse sentido os grupos de homologia com coeficientes locais, que como veremos posteriormente refletem melhor a geometria do espaço, são uma generalização dos grupos de homologia ordinários.

\subsection{Homologia Relativa}

Se $A \subset X$ denotaremos por $i: A \hookrightarrow X$ a inclusão e por $\left.G\right|_{A}$ o feixe $i^{*} G$ induzido por ela. Se $c=\sum g_{j} \mu_{j} \in C_{q}\left(A ;\left.G\right|_{A}\right)$ temos que $\mu_{j}: \Delta^{q} \rightarrow A$ e $g_{j} \in\left(i^{*} G\right)\left(\mu_{j}\left(e_{0}\right)\right)=$ $G\left(\left(i \circ \mu_{j}\right)\left(e_{0}\right)\right)=G\left(\mu_{j}^{\prime}\left(e_{0}\right)\right)$, onde $\mu_{j}^{\prime}=i \circ \mu_{j}: \Delta^{q} \rightarrow X$.

Dessa forma podemos definir um monomorfismo $i_{\sharp}: C_{q}\left(A ;\left.G\right|_{A}\right) \rightarrow C_{q}(X ; G)$ por:

$$
i_{\text {甘 }}(c)=\sum g_{j} \mu_{j}^{\prime} \in C_{q}(X ; G) .
$$

Como $C_{q}\left(A ;\left.G\right|_{A}\right)$ é um subgrupo de $C_{q}(X ; G)$ temos que $C_{*}\left(A ;\left.G\right|_{A}\right)$ é um subcomplexo de cadeia.

Definimos então:

$$
C_{q}(X, A ; G)=\operatorname{coker}\left(i_{\sharp}\right)=\frac{C_{q}(X ; G)}{C_{q}\left(A ;\left.G\right|_{A}\right)}
$$

e temos a seqüência exata curta:

$$
0 \rightarrow C_{*}\left(A ;\left.G\right|_{A}\right) \rightarrow C_{*}(X ; G) \rightarrow C_{*}(X, A ; G) \rightarrow 0 .
$$

Finalmente definindo $H_{q}(X, A ; G)=H_{q}\left(C_{*}(X, A ; G)\right)$ obtemos os grupos de homologia relativos e, consequentemente, a seqüência exata longa em homologia:

$$
\cdots \rightarrow H_{q+1}(X, A ; G) \stackrel{\partial_{*}}{\longrightarrow} H_{q}\left(A ;\left.G\right|_{A}\right) \stackrel{i_{*}}{\longrightarrow} H_{q}(X ; G) \stackrel{j_{*}}{\longrightarrow} H_{q}(X, A ; G) \rightarrow \cdots .
$$


Se $G$ e $H$ são sistemas de coeficientes locais em $X$ e se $\Phi: G \rightarrow H$ é um homomorfismo então $\Phi$ induz um homomorfismo $\Phi_{\sharp}: C_{*}(X ; G) \rightarrow C_{*}(X ; H)$-dado por:

$$
\Phi_{\text {甘 }}\left(\sum g_{i} \mu_{i}\right)=\sum \Phi\left(\mu_{i}\left(e_{0}\right)\right) g_{i} \mu_{i} .
$$

Claramente $\Phi_{\sharp}$ é uma aplicação de cadeia. Além disso se $A \subset X$ então $\left.\Phi_{\sharp}\right|_{C_{*}\left(A ;\left.G\right|_{A}\right)}$ : $C_{*}\left(A ;\left.G\right|_{A}\right) \rightarrow C_{*}\left(A ;\left.H\right|_{A}\right)$ e assim $\Phi$ induz uma aplicação $\Phi_{*}: H_{q}(X, A ; G) \rightarrow$ $H_{q}(X, A ; H)$.

Seja $f:(X, A) \rightarrow(Y, B)$ e $G$ um sistema de coeficientes locais em $Y . f$ induz uma $f_{\sharp}: C_{*}\left(X ; f^{*} G\right) \rightarrow C_{*}(Y ; G)$ que, restrita a $C_{*}\left(A ;\left.f^{*} G\right|_{A}\right)$ tem imagem em $C_{*}\left(B ;\left.G\right|_{B}\right)$.

Se $F$ é um sistema de coeficiente locais em $X$ e $\Phi: F \rightarrow f^{*} G$ é um homomorfismo, podemos compor $\Phi_{\sharp}$ com $f_{\sharp}$ para obter uma aplicação de $C_{*}(X ; F)$ em $C_{*}(Y ; G)$; tal aplicação também pode ser restrita a $C_{*}\left(A ;\left.F\right|_{A}\right)$, ainda com imagem em $C_{*}\left(B ;\left.G\right|_{B}\right)$. Dessa forma a composta acima induz um homomorfismo de $H_{q}(X, A ; F)$ em $H_{q}(Y, B ; G)$.

\subsection{Homologia de um Ponto de Vista Axiomático}

Para traduzir as definições acima para a linguagem categórica precisamos de algumas definições e notações.

Definição 1.21. Um espaço topológico $X$ é Compactamente Gerado quando para qualquer $A \subset X$ que satisfaça: $A \cap C$ é fechado para todo $C \subset X$ compacto, temos que $A$ é fechado em $X$.

Seja $\mathbb{K}$ a categoria cujos elementos são os espaços compactamente gerados e cujos morfismos são as funções contínuas. Definimos $\mathbb{L}$ como a categoria formada pelas triplas $(X, A ; G)$ como elementos, $\operatorname{com}(X, A)$ um par em $\mathbb{K}$ e $G$ é um sistema de coeficientes locais em $X$.

Um morfismo $\phi:(X, A ; G) \rightarrow(Y, B ; H)$ de $\mathbb{L}$ é um par $\left(\phi_{1}, \phi_{2}\right)$ tal que:

- $\phi_{1}:(X, A) \rightarrow(Y, B)$ é uma função contínua entre pares.

- $\phi_{2}: G \rightarrow \phi_{1}^{*} H$ é um homomorfismo de sistemas de coeficientes locais.

Se $\psi:(Y, B ; H) \rightarrow(Z, C ; K)$ é outro morfismo de $\mathbb{L}$ então $\omega=\psi \circ \phi$ também é um morfismo de $\mathbb{L} \operatorname{com} \omega_{1}=\psi_{1} \circ \phi_{1}$ e $\omega_{2}=\left(\phi_{1}^{*} \psi_{2}\right) \circ \phi_{2}$, onde $\phi_{1}^{*} \psi_{2}: \phi_{1}^{*} H \rightarrow \phi_{1}^{*} \psi_{1}^{*} K$ é um homomorfismo tal que:

$$
\phi_{1}^{*} \psi_{2}(x)=\psi_{2}\left(\phi_{1}(x)\right):\left(\phi_{1}^{*} H\right)(x)=H\left(\phi_{1}(x)\right) \rightarrow\left(\phi_{1}^{*} \psi_{1}^{*} K\right)(x)=K\left(\omega_{1}(x)\right) .
$$




\subsubsection{Homotopias em $\mathbb{L}$}

Sejam $i_{t}: X \rightarrow I \times X$ e $p: I \times X \rightarrow X$ dadas por $i_{t}(x)=(t, x)$ e $p(t, x)=x$ temos:

Definição 1.22. O Prisma, $I \times(X, A ; G)$ de um objeto $(X, A ; G)$ de $\mathbb{L}$ é o objeto $\left(I \times X, I \times A ; p^{*} G\right)$ também em $\mathbb{L}$.

Sejam $\phi, \psi:(X, A ; G) \rightarrow(Y, B ; H)$ aplicações em $\mathbb{L}$.

Definição 1.23. Uma Homotopia entre $\phi$ e $\psi$ é uma aplicação $\lambda: I \times(X, A ; G) \rightarrow$ $(Y, B ; H), \lambda=\left(\lambda_{1}, \lambda_{2}\right)$, tal que:

- $\lambda_{1}:(I \times X, I \times A) \rightarrow(Y, B)$ é uma homotopia (ordinária) entre $\phi_{1}$ e $\psi_{1}$.

- $\lambda_{2}: p^{*} G \rightarrow \lambda_{1}^{*} H$ é um homomorfismo de sistemas de coeficientes locais satisfazendo:

1. $i_{0}^{*} \lambda_{2}=\phi_{2}: G \rightarrow \phi^{*} H$.

2. $i_{1}^{*} \lambda_{2}=\phi_{2}: G \rightarrow \phi^{*} H$. elas.

Dizemos que $\phi$ e $\psi$ são homotópicas $(\phi \simeq \psi)$ se existir uma homotopia entre

Proposição 1.24. Homotopia é uma relação de equivalência nos morfismos de $\mathbb{L}$.

Demonstração: Decorre facilmente da definição.

Também é fácil ver que:

Proposição 1.25. Se $\phi \simeq \psi:(X, A ; F) \rightarrow(Y, B ; G)$ e $\phi^{\prime} \simeq \psi^{\prime}:(Y, B ; G) \rightarrow$ $(Z, C ; H)$ então:

$$
\phi^{\prime} \circ \phi \simeq \psi^{\prime} \circ \psi:(X, A ; F) \rightarrow(Z, C ; H)
$$

\subsection{2 "Axiomas"}

Definição 1.26. O Operador "Shift" é um funtor $R: \mathbb{L} \rightarrow \mathbb{L}$ definido nos objetos de $\mathbb{L}$ por:

$$
R(X, A ; G)=\left(A, \varnothing ;\left.G\right|_{A}\right),
$$

e para $\phi:(X, A ; G) \rightarrow(Y, B ; K)$ morfismo $R(\phi)=\psi$ satisfazendo: 
- $\psi_{1}:(X, A) \rightarrow(Y, B)$ é a restrição de $\phi_{1}$. Em outras palavras:

$$
j \circ \psi_{1}=\phi_{1} \circ i
$$

onde $i: A \hookrightarrow X$ e $j: B \hookrightarrow Y$ são as inclusões.

- $\psi_{2}=i^{*} \phi_{2}:\left.G\right|_{A}=i^{*} G \rightarrow i^{*} \phi_{1}^{*} H=\psi_{1}^{*} j^{*} H=\psi_{1}^{*}\left(\left.H\right|_{B}\right)$.

Podemos agora considerar $H_{q}: \mathbb{L} \rightarrow \mathfrak{A}$ como uma seqüência de funtores, além disso temos $\partial_{q}: H_{q} \rightarrow H_{q-1} \circ R$ uma transformação natural. Definindo a inclusão em $\mathbb{L}$ de maneira.natural $\left(\left(X_{1}, A_{1} ; G_{1}\right) \stackrel{k}{\hookrightarrow}(X, A ; G) \operatorname{com} X_{1} \subset X, A_{1} \subset A, k_{1}\right.$ a inclusão natural de $X_{1}$ em $X, G_{1}=\left.G\right|_{X_{1}}$ e $k_{2}:\left.G_{1} \rightarrow G\right|_{X_{1}}$ a identidade) obtemos $H_{q}(k): H_{q}\left(X_{1}, A_{1} ; G_{1}\right) \rightarrow H_{q}(X, A ; G)$ uma injeção. Podemos agora enunciar uma lista de propriedades análogas aos axiomas de Eilenberg-Steenrod.

Teorema 1.27. Os funtores $H_{*}$ e as transformações naturais $\partial_{*}$ satisfazem as seguintes propriedades:

1. Exatidão $S e(X, A ; G)$ é um objeto em $\mathbb{L}$, $i$ e $j$ as inclusões apropriadas então a seqüência:

$\cdots \rightarrow H_{q+1}(X, A ; G) \stackrel{\partial_{q+1}}{\longrightarrow} H_{q}\left(A ;\left.G\right|_{A}\right) \stackrel{H_{q}(i)}{\longrightarrow} H_{q}(X ; G) \stackrel{H_{q}(j)}{\longrightarrow} H_{q}(X, A ; G) \rightarrow \cdots$, é exata.

2. Invariância $S e \phi_{0}, \phi_{1}:(X, A ; G) \rightarrow(Y, B ; H)$ são morfismos homotópicos em $\mathbb{L}$ então

$$
H_{q}\left(\phi_{0}\right)=H_{q}\left(\phi_{1}\right): H_{q}(X, A ; G) \rightarrow H_{q}(Y, B ; H)
$$

para todo $q$.

3. Excisão $S e\left(X ; X_{1}, X_{2}\right)$ é uma tríade em $\mathbb{K}$ tal que $X=\operatorname{Int} X_{1} \cup \operatorname{Int} X_{2}$ e se $G$ é um sistema de coeficientes locais em $X$, então a injeção $H_{q}\left(X_{1}, X_{1} \cap X_{2} ;\left.G\right|_{X_{1}}\right) \rightarrow H_{q}\left(X, X_{2} ; G\right)$ é um isomorfismo para todo $q$.

4. Homologia do Ponto Se $X=\{*\}$, um único ponto, então:

$$
H_{q}(X ; G)=\left\{\begin{array}{lll}
0 & \text { se } & q \neq 0 \\
G(*) & \text { se } & q=0
\end{array}\right.
$$

5. Aditividade $S e X$ é a união disjunta de uma família de abertos $X_{\alpha}$, A um subconjunto de $X, A_{\alpha}=A \cap X_{\alpha}, G$ um sistema de coeficientes locais em $X$ $e G_{\alpha}=\left.G\right|_{X_{\alpha}}$. Então as injeções

$$
H_{q}\left(X_{\alpha}, A_{\alpha} ; G_{\alpha}\right) \rightarrow H_{q}(X, A ; G)
$$

representam $H_{q}(X, A ; G)$ como uma soma direta.

Demonstração: Análoga a demonstração feita no caso ordinário. 


\subsection{Sequência de Mayer-Vietoris}

Para construírmos a sequência de Mayer-Vietoris usaremos dois lemas algébricos:

Lema 1.28. Se no seguinte diagrama de grupos abelianos e homomorfismos:

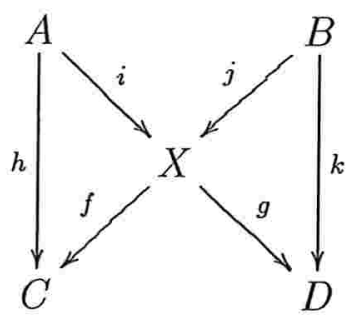

os triângulos forem comutativos, $h$ e $k$ forem isomorfismos, $\operatorname{Im}(i)=\operatorname{Ker}(g) e$ $\operatorname{Im}(j)=\operatorname{Ker}(f)$ então os dois homomorfismos:

$$
\phi: A \oplus B \rightarrow X \quad \text { e } \quad \psi: X \rightarrow C \oplus D
$$

definidos por:

$$
\begin{gathered}
\phi(x, y)=i(x)+j(y), \quad(x, y) \in A \oplus B ; \\
\psi(x)=(f(x), g(x)), \quad x \in X
\end{gathered}
$$

são isomorfismos.

Demonstração: [Hu, I, 7.1,página 39].

Lema 1.29. Se no seguinte diagrama de grupos abelianos e homomorfismos:

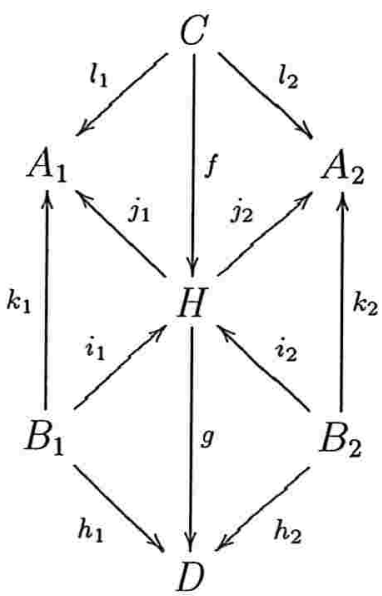

os triângulos forem comutativos, $k_{1}$ e $k_{2}$ forem isomorfismos, $\operatorname{Im}\left(i_{1}\right)=\operatorname{Ker}\left(j_{2}\right)$ e $\operatorname{Im}\left(i_{2}\right)=\operatorname{Ker}\left(j_{1}\right)$ então: 
1. O homomorfismo $i_{1} \circ k_{1}^{-1} \circ j_{1}+i_{2} \circ k_{2}^{-1} \circ j_{2}: H \rightarrow H$ é a identidade.

2. $h_{1} \circ k_{1}^{-1} \circ l_{1}+h_{2} \circ k_{2}^{-1} \circ l_{2}=g \circ f$.

Demonstração: [Hu, II, 9.1, página 106].

Trivialmente temos:

Corolário 1.30. Nas condições acima se go $f=0$ então $-h_{1} \circ k_{1}^{-1} \circ l_{1}=h_{2} \circ k_{2}^{-1} \circ l_{2}$.

Seja $\left(X ; X_{1}, X_{2}\right)$ é uma tríade em $\mathbb{K}$ tal que $X=\operatorname{Int} X_{1} \cup \operatorname{Int} X_{2}$, sendo $C=$ $X_{1} \cap X_{2}$ e $G$ um sistema de coeficientes locais ${ }^{1}$ em $X$ podemos definir, para todo $q$ inteiro:

1. $\psi: H_{q}(C ; G) \rightarrow H_{q}\left(X_{1} ; G\right) \oplus H_{q}\left(X_{2} ; G\right)$ dada por:

$$
\psi(x)=\left(h_{1 *}(x),-h_{2 *}(x)\right)
$$

onde $h_{i *}: H_{q}(C ; G) \rightarrow H_{q}\left(X_{i} ; G\right)$ são os homomorfismos induzidos das inclusões $h_{i}: C \rightarrow X_{i}$.

2. $\phi: H_{q}\left(X_{1} ; G\right) \oplus H_{q}\left(X_{2} ; G\right) \rightarrow H_{q}(X ; G)$ dada por:

$$
\phi(x, y)=m_{1 *}(x)+m_{2 *}(y)
$$

onde $m_{i *}: H_{q}\left(X_{i} ; G\right) \rightarrow H_{q}(X ; G)$ são os homomorfismos induzidos das inclusões $m_{i}: X_{i} \rightarrow X$.

Tomando-se o diagrama:

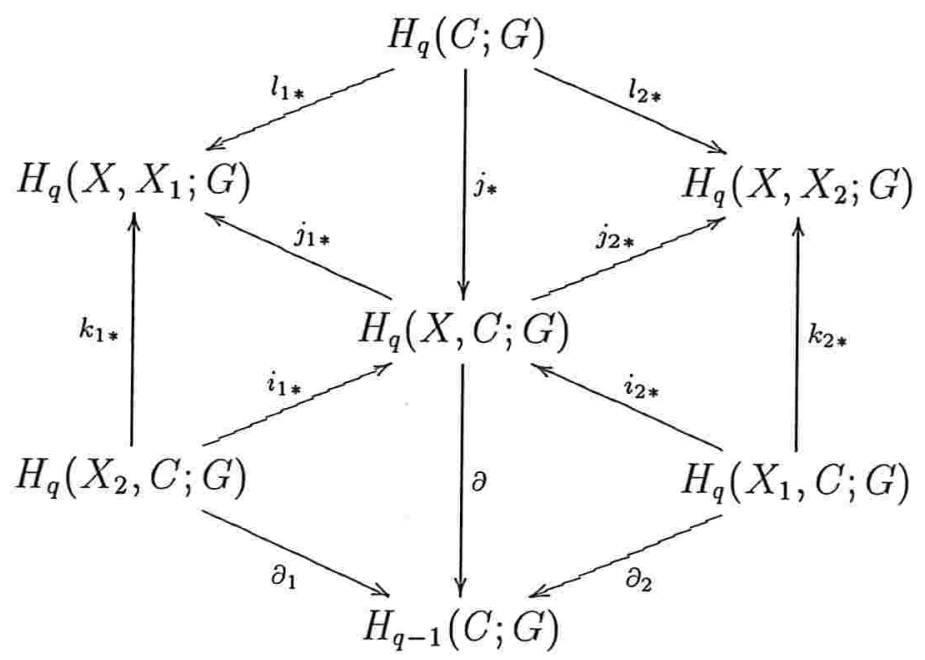

\footnotetext{
${ }^{1}$ Por simplicidade de notação a restrição de $G$ a qualquer subconjunto de $X$ será denotada também como $G$
} 
onde $\partial, \partial_{1}, \partial_{2}$ são operadores bordo e os demais homomorfismos são induzidos das respectivas inclusões podemos, usando-o Lema 1.29, definir um homomorfismo $\Delta: H_{q}(X: G) \rightarrow H_{q-1}(C ; G)$ tomando-se:

$$
\Delta=-\partial_{1} \circ k_{1 *}^{-1} \circ l_{1 *}=\partial_{2} \circ k_{2 *}^{-1} \circ l_{2 *} .
$$

Definição 1.31. A sequência:

$\cdots \rightarrow H_{q}(C ; G) \stackrel{\psi}{\longrightarrow} H_{q}\left(X_{1} ; G\right) \oplus H_{q}\left(X_{2} ; G\right) \stackrel{\phi}{\longrightarrow} H_{q}(X ; G) \stackrel{\Delta}{\longrightarrow} H_{q-1}(C ; G) \rightarrow \cdots$

é chamada Sequência de Mayer-Vietoris da tríade $\left(X ; X_{1}, X_{2}\right)$.

Teorema 1.32. A sequência de Mayer-Vietoris de uma tríade $\left(X ; X_{1}, X_{2}\right)$ é exata.

Demonstração: Notemos que o seguinte diagrama, obtido a partir dos homomorfismos induzidos das inclusões é comutativo:

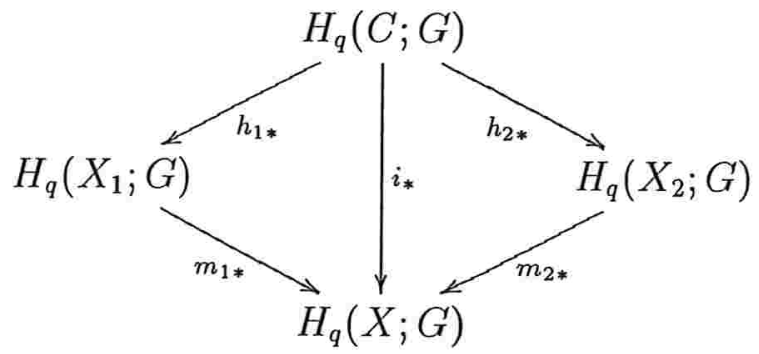

1. A sequência é exata em $H_{q}(X ; G)$

- $\operatorname{Im}(\phi) \subset K \operatorname{Ker}(\Delta)$

Seja $u \in \operatorname{Im}(\phi) \subset H_{q}(X ; G)$, existe $(x, y) \in H_{q}\left(X_{1} ; G\right) \oplus H_{q}\left(X_{2} ; G\right)$ tal que $u=\phi(x, y)=m_{1 *}(x)+m_{2 *}(y)$, assim:

$$
\begin{aligned}
\Delta(u) & =\Delta\left(m_{1 *}(x)+m_{2 *}(y)\right) \\
& =\Delta\left(m_{1 *}(x)\right)+\Delta\left(m_{2 *}(y)\right) \\
& =\left(-\partial_{1} \circ k_{1 *}^{-1} \circ l_{1 *} \circ m_{1 *}\right)(x)+\left(\partial_{2} \circ k_{2 *}^{-1} \circ l_{2 *} \circ m_{2 *}\right)(y) \\
& =0 .
\end{aligned}
$$

pois da sequência dos pares $\left(X, X_{i}\right)$ temos que $l_{i *} \circ m_{i *}=0$.

- $\operatorname{Ker}(\Delta) \subset \operatorname{Im}(\phi)$

Seja $u \in K \operatorname{Ker}(\Delta)$, assim:

$$
\left(-\partial_{1} \circ k_{1 *}^{-1} \circ l_{1 *}\right)(u)=\left(\partial_{2} \circ k_{2 *}^{-1} \circ l_{2 *}\right)(u)=0
$$


Pela exatidão das sequências de homologia dos pares $\left(X_{i}, C\right)$ existem $x \in H_{q}\left(X_{1} ; G\right)$ e $y \in H_{q}\left(X_{2} ; G\right)$ tais que:

$$
n_{1 *}(x)=k_{2 *}^{-1}\left(l_{2 *}(u)\right) \text { e } n_{2 *}(y)=k_{1 *}^{-1}\left(l_{1 *}(u)\right)
$$

onde $n_{i *}: H_{q}\left(X_{i} ; G\right) \rightarrow H_{q}\left(X_{i}, C ; G\right)$ são induzidas das inclusões $n_{i}$ : $X_{i} \rightarrow\left(X_{i}, C\right)$.

Pelo Lema 1.29 o homorfismo $e=i_{1 *} \circ k_{1 *}^{-1} \circ j_{1 *}+i_{2 *} \circ k_{2 *}^{-1} \circ j_{2 *}$ é a identidade de $H_{q}(X ; G)$, assim, notando-se que $i_{1} \circ n_{2}=j \circ m_{2}$ e $i_{2} \circ n_{1}=j \circ m_{1}$, temos que:

$$
\begin{aligned}
j_{*}(u) & =e\left(j_{*}(u)\right) \\
& =\left(i_{1 *} \circ k_{1 *}^{-1} \circ j_{1 *} \circ j_{*}+i_{2 *} \circ k_{2 *}^{-1} \circ j_{2 *} \circ j_{*}\right)(u) \\
& =\left(i_{1 *} \circ k_{1 *}^{-1} \circ j_{1 *} \circ j_{*}\right)(u)+\left(i_{2 *} \circ k_{2 *}^{-1} \circ j_{2 *} \circ j_{*}\right)(u) \\
& =\left(i_{1 *} \circ k_{1 *}^{-1} \circ l_{1 *}\right)(u)+\left(i_{2 *} \circ k_{2 *}^{-1} \circ l_{2 *}\right)(u) \\
& =i_{1 *}\left(n_{2 *}(y)\right)+i_{2 *}\left(n_{1 *}(x)\right) \\
& =j_{*}\left(m_{2 *}(y)\right)+j_{*}\left(m_{1 *}(x)\right)
\end{aligned}
$$

Portanto;

$$
j_{*}\left(u-m_{1 *}(x)-m_{2 *}(y)\right)=0 .
$$

Pela exatidão da sequência de homologia do par $(X, C)$ existe um $w \in$ $H_{q}(C ; G)$ tal que:

$$
i_{*}(w)=u-m_{1 *}(x)-m_{2 *}(y) .
$$

Tomando-se $x^{\prime}=x+h_{1 *}(w)$ obtemos:

$$
\begin{aligned}
\phi\left(x^{\prime}, y\right) & =m_{1 *}\left(x^{\prime}\right)+m_{2 *}(y) \\
& =m_{1 *}(x)+m_{1 *}\left(h_{1 *}(w)\right)+m_{2 *}(y) \\
& =m_{1 *}(x)+i_{*}(w)+m_{2 *}(y) \\
& =u .
\end{aligned}
$$

2. A sequência é exata em $H_{q}\left(X_{1} ; G\right) \oplus H_{q}\left(X_{2} ; G\right)$ :

- $\operatorname{Im}(\psi) \subset \operatorname{Ker}(\phi)$

Seja $(x, y) \in \operatorname{Im}(\psi) \subset H_{q}\left(X_{1} ; G\right) \oplus H_{q}\left(X_{2} ; G\right)$, existe $u \in H_{q}(C ; G)$ tal que $(x, y)=\psi(u)=\left(h_{1 *}(u),-h_{2 *}(u)\right)$. Assim $x=h_{1 *}(u)$ e $y=-h_{2 *}(u)$.

Portanto:

$$
\begin{aligned}
\phi(x, y) & =m_{1 *}(x)+m_{2 *}(y) \\
& =m_{1 *}\left(h_{1 *}(u)\right)-m_{2 *}\left(h_{2 *}(u)\right) \\
& =i_{*}(u)-i_{*}(u)=0 .
\end{aligned}
$$


- $\operatorname{Ker}(\phi) \subset \operatorname{Im}(\psi)$

$\operatorname{Seja}(x, y) \in K \operatorname{Ker}(\phi) \subset H_{q}\left(X_{1} ; G\right) \oplus H_{q}\left(X_{2} ; G\right), \operatorname{assim} \phi(x, y)=m_{1 *}(x)+$ $m_{2 *}(y)=0$

Portanto (usando a notação do ítem anterior):

$$
\begin{aligned}
i_{1 *}\left(n_{2 *}(y)\right)+i_{2 *}\left(n_{1 *}(x)\right) & =j_{*}\left(m_{2 *}(y)\right)+j_{*}\left(m_{1 *}(x)\right) \\
& =j_{*}\left(m_{2 *}(y)+m_{1 *}(x)\right) \\
& =0 .
\end{aligned}
$$

Usando o Lema $1.28^{2}$ temos que o homomorfismo:

$$
i_{1 *}+i_{2 *}: H_{q}\left(X_{2} ; G\right) \oplus H_{q}\left(X_{1} ; G\right) \rightarrow H_{q}(X ; G)
$$

é um isomorfismo e portanto:

$$
n_{1 *}(x)=0 \text { e } m_{2 *}(y)=0 .
$$

Pela exatidão das sequências de homologia dos pares $\left(X_{i}, C\right)$ existem $w, v \in H_{q}(C ; G)$ tais que:

$$
h_{1 *}(w)=x \text { e } h_{2 *}(v)=y .
$$

Temos então que:

$$
\begin{aligned}
0 & =\phi(x, y) \\
& =m_{1 *}\left(h_{1 *}(w)\right)+m_{2 *}\left(h_{2 *}(v)\right) \\
& =i_{*}(w+v)
\end{aligned}
$$

Pela exatidão da sequência do par $(X, C)$ existe $t \in H_{q+1}(X, C ; G)$ com $\partial(t)=w+v$. Novamente usando o Lema 1.28 existem $x^{\prime} \in H_{q}\left(X_{1}, C ; G\right)$ e $y^{\prime} \in H_{q}\left(X_{2}, C ; G\right)$ tais que:

$$
t=i_{1 *}\left(y^{\prime}\right)+i_{2 *}\left(x^{\prime}\right) .
$$

${ }^{2}$ Podemos montar o seguinte diagrama:

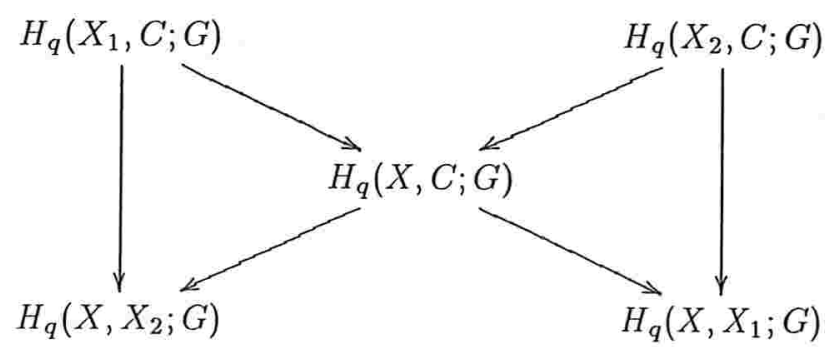

com os homomorfismos induzidos das inclusões 
Assim:

$$
\begin{aligned}
w+v & =\partial(t) \\
& =\partial\left(i_{1 *}\left(y^{\prime}\right)+i_{2 *}\left(x^{\prime}\right)\right) \\
& =\partial_{1}\left(y^{\prime}\right)+\partial_{2}\left(x^{\prime}\right) .
\end{aligned}
$$

Tomando-se $s=w-\partial_{2}\left(x^{\prime}\right)=-\left[v-\partial_{1}\left(y^{\prime}\right)\right]$ obtemos:

$$
\begin{aligned}
\psi(s) & =\left(h_{1 *}(s),-h_{2 *}(s)\right) \\
& =\left(h_{*}(w)-h_{1 *}\left(\partial_{2}\left(x^{\prime}\right)\right),-\left[-h_{2 *}(v)+h_{2 *}\left(\partial_{1}\left(y^{\prime}\right)\right)\right]\right) \\
& =\left(h_{1 *}(w), h_{2 *}(v)\right) \\
& =(x, y) .
\end{aligned}
$$

3. A sequência é exata em $H_{q-1}(C ; G)$

- $\operatorname{Im}(\Delta) \subset \operatorname{Ker}(\psi)$

Seja $x \in \operatorname{Im}(\Delta) \subset H_{q-1}(C ; G)$, assim existe $w \in H_{q}(X ; G)$ com $\Delta(w)=$ $x$.

Pela exatidão das sequências dos pares $\left(X_{i}, C\right)$ temos que $h_{1 *} \circ \partial_{2}=0$ e $h_{2 *} \circ \partial_{1}=0$ portanto:

$$
\begin{aligned}
\psi(x) & =\left(h_{1 *}(x),-h_{2 *}(x)\right) \\
& =\left(h_{1 *}(\Delta(w)),-h_{2 *}(\Delta(w))\right) \\
& =\left(\left(h_{1 *} \circ \partial_{2} \circ k_{2 *}^{-1} \circ l_{2 *}\right)(w),-\left(h_{2 *} \circ \partial_{1} \circ k_{1 *}^{-1} \circ l_{1 *}\right)(w)\right. \\
& =(0,0)
\end{aligned}
$$

- $\operatorname{Ker}(\psi) \subset \operatorname{Im}(\Delta)$

Seja $x \in K \operatorname{Ker}(\psi)$ assim $h_{1 *}(x)=h_{2 *}(x)=0$. Pela exatidão das sequências dos pares $\left(X_{i}, C\right)$ existem $a_{i} \in H_{q}\left(X_{i}, C ; G\right)$ tais que:

$$
\partial_{1}\left(a_{2}\right)=x \text { e } \partial_{2}\left(a_{1}\right)=-x .
$$

Logo

$$
\begin{aligned}
\partial\left(i_{1 *}\left(a_{2}\right)+i_{2 *}\left(a_{1}\right)\right) & =\partial\left(i_{1 *}\left(a_{2}\right)\right)+\partial\left(i_{2 *}\left(a_{1}\right)\right) \\
& =\partial_{1}\left(a_{2}\right)+\partial_{2}\left(a_{1}\right) \\
& =x-x \\
& =0
\end{aligned}
$$

Pela exatidão da sequência de homologia do par $(X, C)$ existe $w \in$ $H_{q}(X ; G)$ tal que:

$$
j_{*}(w)=-i_{1 *}\left(a_{2}\right)-i_{2 *}\left(a_{1}\right)
$$


Agora, notando-se que $j_{1 *} \circ i_{1 *}=k_{1 *}$ e $j_{1 *} \circ i_{2 *}=0$, temos que:

$$
\begin{aligned}
\Delta(w) & =-\left(\partial_{1} \circ k_{1 *}^{-1} \circ l_{1 *}\right)(w) \\
& =-\left(\partial_{1} \circ k_{1 *}^{-1} \circ j_{1 *} \circ j_{*}\right)(w) \\
& =\left(\partial_{1} \circ k_{1 *}^{-1} \circ j_{1 *}\right)\left(i_{1 *}\left(a_{2}\right)+i_{2 *}\left(a_{1}\right)\right) \\
& =\left(\partial_{1} \circ k_{1 *}^{-1} \circ j_{1 *} \circ i_{1 *}\right)\left(a_{2}\right)+\left(\partial_{1} \circ k_{1 *}^{-1} \circ j_{1 *} \circ i_{2 *}\right)\left(a_{1}\right) \\
& =\left(\partial_{1} \circ k_{1 *}^{-1} \circ k_{1 *}\right)\left(a_{2}\right)+0 \\
& =\partial_{1}\left(a_{2}\right) \\
& =x
\end{aligned}
$$

Observação 1.33. Note que nesse caso $H_{1}\left(X_{1} \vee X_{2} ; G\right)$ não necessariamente é isomorfo a $H_{1}\left(X_{1} ;\left.G\right|_{X_{1}}\right) \oplus H_{1}\left(X_{2} ;\left.G\right|_{X_{2}}\right)$. Como exemplo podemos tomar a figura oito, $S^{1} \vee S^{1}, \operatorname{com} G \approx \mathbb{Z}$ e a ação de cada um dos geradores de $\pi_{1}\left(S^{1} \vee S^{1}\right)$ como $o$ isomorfismo (-id). Nessa situação obtemos $H_{1}\left(X_{1} ;\left.G\right|_{X_{1}}\right)=H_{1}\left(X_{2} ;\left.G\right|_{X_{2}}\right)=$ $H_{1}\left(S^{1} ;\left.G\right|_{S^{1}}\right)=0$ e $H_{1}\left(X_{1} \vee X_{2} ; G\right)=\mathbb{Z}$.

\subsection{Cohomologia com Coeficientes Locais}

Definimos $C^{n}(X ; G)$ como o conjunto de todas as funções $c$ que associam a cada simplexo singular $\mu: \Delta^{n} \rightarrow X$ um elemento $c(\mu) \in G\left(\mu\left(e_{0}\right)\right)$. Claramente $C^{n}(X ; G)$ é um grupo abeliano com a soma usual de funções pois $G\left(\mu\left(e_{0}\right)\right)$ é abeliano.

O Operador Cobordo $\delta: C^{n}(X ; G) \rightarrow C^{n+1}(X ; G)$ é definido por:

$$
(-1)^{n} \delta c(u)=G\left(\sigma_{\mu}\right)^{-1} c\left(\partial_{0} \mu\right)+\sum_{i=1}^{n+1}(-1)^{i} c\left(\partial_{i} \mu\right)
$$

para cada $n+1$-simplexo singular $\mu$.

$\delta$ é um homomorfismo e $\delta$ o $\delta=0$ de modo que $C^{*}(X ; G)$ é um complexo de co-cadeia.

Se $A \subset X$, a restrição $i^{\sharp}: C^{*}(X ; G) \rightarrow C^{*}\left(A ;\left.G\right|_{A}\right)$ é sobrejetora e definimos $C^{*}(X, A ; G)$ como o núcleo de $i^{\sharp}$. $C^{*}(X, A ; G)$ é um sub-complexo de $C^{*}(X ; G)$ e assim podemos definir:

$$
H^{n}(X, A ; G)=H^{n}\left(C^{*}(X, A ; G)\right) \text {. }
$$




\subsubsection{Cohomologia de um Ponto de Vista Axiomático}

Se $\mathbb{L}^{*}$ é a categoria cujos elementos são triplas $(X, A ; G)$, onde $(X, A)$ é um par em $\mathbb{K}$ e $G$ é um sistema de coeficientes locais em $X$ (mesmos elementos de $\mathbb{L}$ ). Um morfismo $\phi:(X, A ; G) \rightarrow(Y, B ; H)$ em $\mathbb{L}^{*}$ é um par $\left(\phi_{1}, \phi_{2}\right)$ tal que:

- $\phi_{1}:(X, A) \rightarrow(Y, B)$ é uma aplicação de pares,

- $\phi_{2}: \phi_{1}^{*} H \rightarrow G$ é um homomorfismo de sistemas de coeficientes locais. $\phi_{2}$ :

Vale a pena ressaltar que a diferença entre $\mathbb{L}$ e $\mathbb{L}^{*}$ é o "sentido" do homomorfismo

- $\operatorname{Em} \mathbb{L}, \phi_{2}: G \rightarrow \phi_{1}^{*} H$,

- $\mathrm{Em} \mathbb{L}^{*}, \phi_{2}: \phi_{1}^{*} H \rightarrow G$.

Se $\phi:(X, A ; G) \rightarrow(Y, B ; H)$ e $\psi:(Y, B ; H) \rightarrow(Z, C ; K)$ são morfismo em $\mathbb{L}^{*}$ temos que $\omega=\psi \circ \phi$ é um morfismo de $\mathbb{L}^{*}$ tal que:

1. $\omega_{1}=\psi_{1} \circ \phi_{1}:(X, A) \rightarrow(Z, C)$,

2. $\omega_{2}(x)=\phi_{2}(x) \circ \psi_{2}\left(\phi_{1}(x)\right): \phi_{1}^{*} \psi_{1}^{*} K \rightarrow G$.

Novamente temos que $\phi:(X, A ; G) \rightarrow(Y, B ; H)$ induz uma aplicação de cocadeia $\phi^{\sharp}: C^{*}(Y, B ; H) \rightarrow C^{*}(X, A ; G)$ dada por:

$$
\phi^{\sharp} c(\mu)=\phi_{2}\left(\mu\left(e_{0}\right)\right)\left(c\left(\phi_{1}(\mu)\right)\right) .
$$

Que, por sua vez, induz:

$$
\phi^{*}=H^{n}(\phi): H^{n}(Y, B ; H) \rightarrow H^{n}(X, A ; G) .
$$

Assim $H^{*}: \mathbb{L}^{*} \rightarrow \mathfrak{A}$ são funtores contra-variantes. Além disso existem transformações naturais ("operador cobordo"):

$$
\delta_{n}: H^{n}\left(A ;\left.G\right|_{A}\right) \rightarrow H^{n+1}(X, A ; G) .
$$

De maneira análoga da vista em homologia, a inclusão induz um homomorfismo: $k^{*}: H^{n}(X, A ; G) \rightarrow H^{n}\left(X_{1}, A_{1} ;\left.G\right|_{X_{1}}\right)$, com $X_{1} \subset X$ e $A_{1} \subset A$. Também de maneira análoga definimos um prisma sobre elementos de $\mathbb{L}^{*}$ e a noção de homotopia em $\mathbb{L}^{*}$.

Obtemos novamente: 
Teorema 1.34. Os funtores $H^{q}$ e as transformações $\delta_{q}$ satisfazem:

1. Exatidão $S e(X, A ; G)$ é um objeto em $\mathbb{L}^{*}$, $i$ e $j$ as inclusões apropriadas então a seqüência:

$\cdots \rightarrow H^{q-1}\left(A ;\left.G\right|_{A}\right) \stackrel{\delta_{q-1}}{\longrightarrow} H^{q}(X, A ; G) \stackrel{H^{q}(j)}{\longrightarrow} H^{q}(X ; G) \stackrel{H^{q}(i)}{\longrightarrow} H^{q}\left(A ;\left.G\right|_{A}\right) \rightarrow \cdots$

é exata.

2. Invariância $S e \phi^{0}$ e $\phi^{1}:(X, A ; G) \rightarrow(Y, B ; H)$ são homotópicas em $\mathbb{L}^{*}$ então:

$$
H^{q}\left(\phi^{0}\right)=H^{q}\left(\phi^{1}\right): H^{q}(Y, B ; H) \rightarrow H^{q}(X, A ; G)
$$

para todo $q$.

3. Excisão $S e\left(X ; X_{1}, X_{2}\right)$ é uma tríade em $\mathbb{K}$ tal que $X=\operatorname{int}\left(X_{1}\right) \cup \operatorname{int}\left(X_{2}\right)$ e se $G$ é um sistema de coeficientes locais em $X$, então:

$$
k^{*}: H^{q}\left(X, X_{2} ; G\right) \rightarrow H^{q}\left(X_{1}, X_{1} \cap X_{2} ;\left.G\right|_{X_{1}}\right)
$$

é um isomorfismo para todo $q$.

4. Cohomologia do ponto $S e X=\{*\}$, então:

$$
H^{q}(X ; G)=\left\{\begin{array}{lll}
0 & \text { se } & q \neq 0 \\
G(*) & \text { se } & q=0
\end{array}\right.
$$

5. Aditividade $S e X$ é a união disjunta de subespaços $X_{\alpha}, A \subset X, A_{\alpha}=$ $A \cap X_{\alpha}, G$ um sistema de coeficientes locais em $X$ e $G_{\alpha}=\left.G\right|_{X_{\alpha}}$, então as injeções:

$$
H^{n}(X, A ; G) \rightarrow H^{n}\left(X_{\alpha}, A_{\alpha} ;\left.G\right|_{X_{\alpha}}\right)
$$

são tais que $H^{n}(X, A ; G)=\prod H^{n}\left(X_{\alpha}, A_{\alpha} ;\left.G\right|_{X_{\alpha}}\right)$.

\subsection{Alguns Exemplos}

Para a realização de cálculos vamos utilizar a formulação celular da teoria conforme [Whitehead, Capítulo VI, seção 4, página 281]. Assim vamos calcular de maneira explícita os grupos de homologia e cohomologia do plano projetivo tanto com coeficientes usuais (em $\mathbb{Z}$ e $\mathbb{Q}$ ) como com coeficientes locais induzidos da orientação local e os grupos de homologia da Garrafa de Klein com os diversos sistemas de coeficientes possíveis para valores em $\mathbb{Z}$. 


\subsubsection{O Plano Projetivo}

Observamos que o plano projetivo é obtido a partir do disco $D^{2}$ com seu bordo identificado.

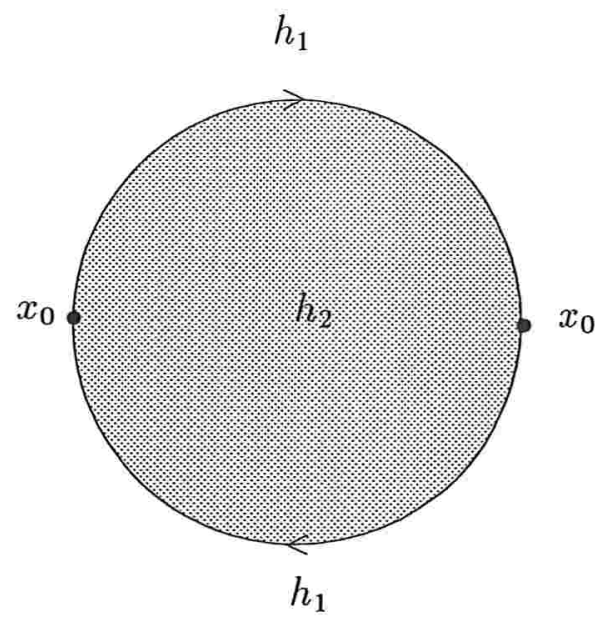

Figura 1.1: Plano Projetivo

Assim podemos construí-lo com:

- Uma 0-célula: $e^{0}: \Delta^{0} \rightarrow x_{0}$;

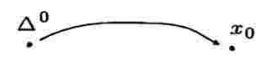

- Uma 1-célula: $e^{1}:\left(\Delta^{1}, \partial \Delta_{1}\right) \rightarrow\left(h_{1}, x_{0}\right)$;

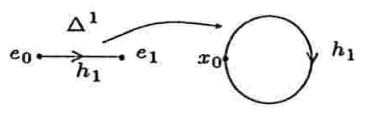

- Uma 2-célula: $e^{2}:\left(\Delta^{2}, \partial \Delta_{2}\right) \rightarrow\left(h_{2}, h_{1}\right)$.

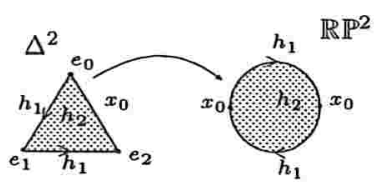

Denotando por $\widetilde{\mathbb{Z}}$ o sistema de coeficientes induzido da orientação temos que:

$$
\begin{gathered}
C_{i}\left(\mathbb{R} \mathbb{P}^{2} ; \mathbb{Z}\right)=<\overline{e^{i}}>\text { onde } \overline{e^{i}}\left(e^{i}\right)=1 \in \mathbb{Z} \\
\text { e } \\
C_{i}\left(\mathbb{R} \mathbb{P}^{2} ; \widetilde{\mathbb{Z}}\right)=<\widetilde{e^{i}}>\text { onde } \widetilde{e^{i}}\left(e^{i}\right)=1 \in \mathbb{Z}_{x_{0}}
\end{gathered}
$$

No caso de coeficientes usuais temos os seguintes bordos:

- $\partial \overline{e^{0}}=0$; 
- $\partial \overline{e^{1}}=\overline{e^{0}}-\overline{e^{0}}=0$;

- $\partial \overline{e^{2}}=\sum_{i=0}^{2}(-1)^{i} \partial_{i} \overline{e^{2}}=\overline{e^{1}}-0+\overline{e^{1}}=2 \overline{e^{1}}$.

Assim:

- $H_{0}\left(\mathbb{R} \mathbb{P}^{2} ; \mathbb{Z}\right)=\mathbb{Z}$

- $H_{1}\left(\mathbb{R} \mathbb{P}^{2} ; \mathbb{Z}\right)=\frac{\mathbb{Z}}{2 \mathbb{Z}}=\mathbb{Z}_{2}$

- $H_{2}\left(\mathbb{R} \mathbb{P}^{2} ; \mathbb{Z}\right)=0$

Já no caso de coeficientes locais induzidos da orientação de $\mathbb{R} \mathbb{P}^{2}$ temos:

- $\partial \widetilde{e^{0}}=0$;

- $\partial \widetilde{e^{1}}=\widetilde{e^{0}}-h_{1}\left(\widetilde{e^{0}}\right)=\widetilde{e^{0}}+\widetilde{e^{0}}=2 \widetilde{e^{0}}$;

- $\partial \widetilde{e^{2}}=h_{1}^{-1}\left(\widetilde{e^{1}}\right)-0+\widetilde{e^{1}}=-\widetilde{e^{1}}+\widetilde{e^{1}}=0$.

Assim:

- $H_{0}\left(\mathbb{R} \mathbb{P}^{2} ; \widetilde{\mathbb{Z}}\right)=\frac{\mathbb{Z}}{2 \mathbb{Z}}=\mathbb{Z}_{2}$;

- $H_{1}\left(\mathbb{R} \mathbb{P}^{2} ; \widetilde{\mathbb{Z}}\right)=0$

- $H_{2}\left(\mathbb{R} \mathbb{P}^{2} ; \widetilde{\mathbb{Z}}\right)=\mathbb{Z}$

De maneira absolutamente análoga obtemos que:

- $H_{0}\left(\mathbb{R} \mathbb{P}^{2} ; \mathbb{Q}\right)=\mathbb{Q}$ e $H_{0}\left(\mathbb{R} \mathbb{P}^{2} ; \widetilde{\mathbb{Q}}\right)=0 ;$

- $H_{1}\left(\mathbb{R} \mathbb{P}^{2} ; \mathbb{Q}\right)=0$ e $H_{1}\left(\mathbb{R} \mathbb{P}^{2} ; \widetilde{\mathbb{Q}}\right)=0 ;$

- $H_{2}\left(\mathbb{R} \mathbb{P}^{2} ; \mathbb{Q}\right)=0$ e $H_{2}\left(\mathbb{R} \mathbb{P}^{2} ; \widetilde{\mathbb{Q}}\right)=\mathbb{Q}$.

Para o cálculo dos grupos de cohomologia temos que:

$$
C^{i}\left(\mathbb{R} \mathbb{P}^{2} ; \mathbb{Z}\right)=<e_{*}^{i}>\text { onde } e_{*}^{i}\left(e^{i}\right)=1 \in \mathbb{Z}
$$




$$
C^{i}\left(\mathbb{R} \mathbb{P}^{2} ; \widetilde{\mathbb{Z}}\right)=<e_{\#}^{i}>\text { onde } e_{\#}^{i}\left(e^{i}\right)=1 \in \mathbb{Z}_{x_{0}}
$$

com os seguintes cobordos:

- Coeficientes usuais

$$
\begin{aligned}
& -\delta e_{*}^{0}\left(e^{1}\right)=\sum_{i=0}^{1} e_{*}^{0}\left(e^{0}\right)=1-1=0 ; \\
& -\delta e_{*}^{1}\left(e^{2}\right)=-\sum_{i=0}^{2}(-1)^{i} e_{*}^{1}\left(\partial_{i} e^{2}\right)=-\left[e_{*}^{1}\left(e^{1}\right)-0+e_{*}^{1}\left(e^{1}\right)\right]=-2 ; \\
& -\delta e_{*}^{2}=0 .
\end{aligned}
$$

- Coeficientes locais

$-\delta e_{\#}^{0}\left(e^{1}\right)=h_{1}^{-1}\left(\partial_{0} e^{1}\right)-\partial_{1} e^{1}=-1-1=-2$;

$-\delta e_{\#}^{1}\left(e^{2}\right)=-\left[h_{1}^{-1}\left(\partial_{0} e^{2}\right)-0+\partial_{2} e^{2}\right]=-\left[-e^{1}+e^{1}\right]=0$;

$-\delta e_{\#}^{2}=0$.

Obtemos então:

- Coeficientes usuais

- $H^{0}\left(\mathbb{R} \mathbb{P}^{2} ; \mathbb{Z}\right)=\mathbb{Z}$

- $H^{1}\left(\mathbb{R} \mathbb{P}^{2} ; \mathbb{Z}\right)=0$

- $H^{2}\left(\mathbb{R} \mathbb{P}^{2} ; \mathbb{Z}\right)=\mathbb{Z}_{2}$

- Coeficientes locais

- $H^{0}\left(\mathbb{R} \mathbb{P}^{2} ; \widetilde{\mathbb{Z}}\right)=0 ;$

- $H^{1}\left(\mathbb{R} \mathbb{P}^{2} ; \widetilde{\mathbb{Z}}\right)=\mathbb{Z}_{2}$

- $H^{2}\left(\mathbb{R} P^{2} ; \widetilde{\mathbb{Z}}\right)=\mathbb{Z}$

Mais uma vez de maneira absolutamente análoga obtemos:

- $H^{0}\left(\mathbb{R P}^{2} ; \mathbb{Q}\right)=\mathbb{Q}$ e $H^{0}\left(\mathbb{R} \mathbb{P}^{2} ; \widetilde{\mathbb{Q}}\right)=0 ;$

- $H^{1}\left(\mathbb{R} \mathbb{P}^{2} ; \mathbb{Q}\right)=0$ e $H^{1}\left(\mathbb{R} \mathbb{P}^{2} ; \widetilde{\mathbb{Q}}\right)=0 ;$

- $H^{2}\left(\mathbb{R} \mathbb{P}^{2} ; \mathbb{Q}\right)=0$ e $H^{2}\left(\mathbb{R} \mathbb{P}^{2} ; \widetilde{\mathbb{Q}}\right)=\mathbb{Q}$. 


\subsubsection{A Garrafa de Klein}

Denotaremos por $K$ a garrafa de Klein temos que $K$ pode ser obtida a partir de um retângulo com os lados identificados:

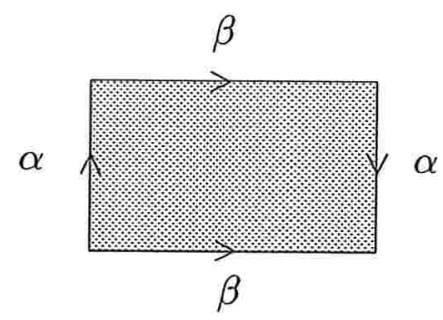

Figura 1.2: Garrafa de Klein

Para construir uma decomposição celular conveniente dividiremos $K$ conforme a figura 1.3:

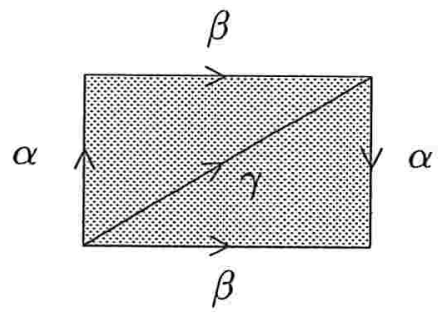

Figura 1.3: Divisão da Garrafa de Klein

Construímos, então, uma decomposição celular com:

- Uma 0-célula: $e^{0}: \Delta^{0} \rightarrow x_{0}$;

- Três 1-células:

$$
\begin{aligned}
& -e_{1}^{1}:\left(\Delta^{1}, \partial \Delta_{1}\right) \rightarrow\left(\alpha, x_{0}\right) ; \\
& -e_{2}^{1}:\left(\Delta^{1}, \partial \Delta_{1}\right) \rightarrow\left(\beta, x_{0}\right) ; \\
& -e_{3}^{1}:\left(\Delta^{1}, \partial \Delta_{1}\right) \rightarrow\left(\gamma, x_{0}\right) ;
\end{aligned}
$$

- Duas 2-células:

$$
\begin{aligned}
& -e_{1}^{2}:\left(\Delta^{2}, \partial \Delta_{2}\right) \rightarrow\left(h_{1}, \alpha \cup \beta \cup \gamma\right) . \\
& -e_{2}^{2}:\left(\Delta^{2}, \partial \Delta_{2}\right) \rightarrow\left(h_{2}, \alpha \cup \beta \cup \gamma\right) .
\end{aligned}
$$




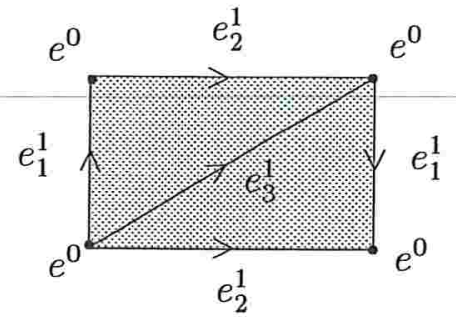

Figura 1.4: Decomposição Celular da Garrafa de Klein

Que podemos representar pela figura:

De maneira genérica denotaremos por $\overline{e^{i}}$ os geradores dos grupos $C_{i}(K ; *)$ tais que: $\overline{e_{j}^{i}}(a)=\left\{\begin{array}{ccc}1 & \text { se } & a=e_{j}^{i} \\ 0 & & \text { c.c. }\end{array}\right.$ para todos os sistemas de coeficientes que usaremos, analogamente $e_{j *}^{i}$ denotará o gerador de $C^{i}(K ; *)$ que satisfaz $e_{j *}^{i}(a)=$ $\left\{\begin{array}{ccc}1 & \text { se } & a=e_{j}^{i} \\ 0 & & \text { c.c. }\end{array}\right.$ denotaremos ainda $\alpha^{*} \beta^{*}$ e $\gamma^{*}$ os isomorfismos induzidos por essas curvas ${ }^{3}$ nos grupos associados aos seus pontos bases pelo sistema de coeficientes locais.

Com a notação acima temos os seguintes bordos:

- $\partial \overline{e^{0}}=0$

- $\quad-\partial \overline{e_{1}^{1}}=\alpha^{*}\left(\overline{e^{0}}\right)-\overline{e^{0}}$;

$-\partial \overline{e_{2}^{1}}=\beta^{*}\left(\overline{e^{0}}\right)-\overline{e^{0}}$;

$-\partial \overline{e_{3}^{1}}=\gamma^{*}\left(\overline{e^{0}}\right)-\overline{e^{0}}$

- $\quad-\partial \overline{e_{1}^{2}}=\alpha^{*}\left(\overline{e_{2}^{1}}\right)-\overline{e_{3}^{1}}+\overline{e_{1}^{1}}$;

$-\partial \overline{e_{2}^{2}}=\gamma^{*}\left(\overline{e_{1}^{1}}\right)-\overline{e_{2}^{1}}+\overline{e_{3}^{1}}$.

E co-bordos:

- $\quad-\delta e_{*}^{0}\left(\overline{e_{1}^{1}}\right)=\alpha^{*-1}\left(e_{*}^{0}\left(\overline{e^{0}}\right)\right)-e_{*}^{0}\left(\overline{e^{0}}\right) ;$

$-\delta e_{*}^{0}\left(\overline{e_{2}^{1}}\right)=\beta^{*-1}\left(e_{*}^{0}\left(\overline{e^{0}}\right)\right)-e_{*}^{0}\left(\overline{e^{0}}\right)$;

$-\delta e_{*}^{0}\left(\overline{e_{3}^{1}}\right)=\gamma^{*-1}\left(e_{*}^{0}\left(\overline{e^{0}}\right)\right)-e_{*}^{0}\left(\overline{e^{0}}\right)$;

- $\quad-\quad * \delta e_{1 *}^{1}\left(\overline{e_{1}^{2}}\right)=-\alpha^{*}\left(e_{1 *}^{1}\left(\overline{e_{2}^{1}}\right)\right)+e_{1 *}^{1}\left(\overline{e_{3}^{1}}\right)-e_{1 *}^{1}\left(\overline{e_{1}^{1}}\right)$;

$* \delta e_{1 *}^{1}\left(\overline{e_{2}^{2}}\right)=-\gamma^{*}\left(e_{1 *}^{1}\left(\overline{e_{1}^{1}}\right)\right)+e_{1 *}^{1}\left(\overline{e_{2}^{1}}\right)-e_{1 *}^{1}\left(\overline{e_{3}^{1}}\right)$;

${ }^{3} \mathrm{Da}$ divisão feita temos que $\gamma \sim \alpha \beta$, assim $\gamma^{*}=\alpha^{*} \circ \beta^{*}$ 


$$
\begin{aligned}
-\quad * \delta e_{2 *}^{1}\left(\overline{e_{1}^{2}}\right) & =-\alpha^{*}\left(e_{2 *}^{1}\left(\overline{e_{2}^{1}}\right)\right)+e_{2 *}^{1}\left(\overline{e_{3}^{1}}\right)-e_{2 *}^{1}\left(\overline{e_{1}^{1}}\right) ; \\
* \delta e_{2 *}^{1}\left(\overline{e_{2}^{2}}\right) & =-\gamma^{*}\left(e_{2 *}^{1}\left(\overline{e_{1}^{1}}\right)\right)+e_{2 *}^{1}\left(\overline{e_{2}^{1}}\right)-e_{2 *}^{1}\left(\overline{e_{3}^{1}}\right) ; \\
-\quad * \delta e_{3 *}^{1}\left(\overline{e_{1}^{2}}\right) & =-\alpha^{*}\left(e_{3 *}^{1}\left(\overline{e_{2}^{1}}\right)\right)+e_{3 *}^{1}\left(\overline{e_{3}^{1}}\right)-e_{3 *}^{1}\left(\overline{e_{1}^{1}}\right) ; \\
* \delta e_{3 *}^{1}\left(\overline{e_{2}^{2}}\right) & =-\gamma^{*}\left(e_{3 *}^{1}\left(\overline{e_{1}^{1}}\right)\right)+e_{3 *}^{1}\left(\overline{e_{2}^{1}}\right)-e_{3 *}^{1}\left(\overline{e_{3}^{1}}\right) ; \\
-\delta e_{1}^{2} & =0 \\
-\delta e_{2}^{2} & =0 .
\end{aligned}
$$

Como dito anteriormente calcularemos os grupos de homologia e cohomologia de $K$ para sistemas com valores em $\mathbb{Z}$, como $\gamma^{*}=\alpha^{*} \circ \beta^{*}$, tais sistemas são descritos por $\alpha^{*}$ e por $\beta^{*}$. Em todos os casos os cálculos serão feitos a partir das sequências:

$$
0 \rightarrow H_{2}\left(K, K^{1} ; * \stackrel{\phi}{\longrightarrow}\right) H_{1}\left(K^{1}, K^{0} ; *\right) \stackrel{\psi}{\longrightarrow} H_{0}\left(K^{0} ; *\right) \rightarrow 0
$$

para homologia e

$$
0 \rightarrow H^{0}\left(K^{0} ; *\right) \stackrel{\varphi}{\longrightarrow} H^{1}\left(K^{1}, K^{0} ; *\right) \stackrel{\theta}{\longrightarrow} H^{2}\left(K^{2}, K^{1} ; *\right) \rightarrow 0
$$

em cohomologia.

Pela decomposição celular que fizemos tais sequências se escrevem como:

$$
0 \rightarrow \mathbb{Z} \oplus \mathbb{Z} \stackrel{\phi}{\longrightarrow} \mathbb{Z} \oplus \mathbb{Z} \oplus \mathbb{Z} \stackrel{\psi}{\longrightarrow} \mathbb{Z} \rightarrow 0
$$

para homologia e

$$
0 \rightarrow \mathbb{Z} \stackrel{\varphi}{\longrightarrow} \mathbb{Z} \oplus \mathbb{Z} \oplus \mathbb{Z} \stackrel{\theta}{\longrightarrow} \mathbb{Z} \oplus \mathbb{Z} \rightarrow 0
$$

em cohomologia.

1. Sistema Trivial $\left(\alpha^{*}=\beta^{*}=i d\right)$ denotado $\mathbb{Z}$.

Temos que:

- $\quad-\phi(1,0)=(1,1,-1)$;

- $\phi(0,1)=(1,-1,1)$;

- $\quad-\psi(1,0,0)=0$;

$-\psi(0,1,0)=0$;

$-\psi(0,0,1)=0$

Portanto:

- $H_{0}(K ; \mathbb{Z})=\mathbb{Z}$ 
- $H_{1}(K ; \mathbb{Z})=\mathbb{Z} \oplus \mathbb{Z}_{2}$;

- $H_{2}(K ; \mathbb{Z})=0$.

Temos ainda:

- $\varphi(1)=(0,0,0)$;

- $\quad-\theta(1,0,0)=(-1,-1)$;

$-\theta(0,1,0)=(-1,1)$;

$-\theta(0,0,1)=(1,-1)$;

Portanto:

- $H^{0}(K ; \mathbb{Z})=\mathbb{Z}$

- $H^{1}(K ; \mathbb{Z})=\mathbb{Z}$;

- $H^{2}(K ; \mathbb{Z})=\mathbb{Z}_{2}$.

2. Sistema dado pela orientação de $K\left(\alpha^{*}=i d\right.$ e $\left.\beta^{*}=-i d\right)$, denotado $\widetilde{\mathbb{Z}}$ :

Temos que:

- $\quad-\phi(1,0)=(1,1,-1)$;

$-\phi(0,1)=(-1,-1,1)$;

- $-\psi(1,0,0)=0$;

$-\psi(0,1,0)=-2$;

$-\psi(0,0,1)=-2$;

Portanto:

- $H_{0}(K ; \widetilde{\mathbb{Z}})=\mathbb{Z}_{2}$

- $H_{1}(K ; \widetilde{\mathbb{Z}})=\mathbb{Z}$

- $H_{2}(K ; \widetilde{\mathbb{Z}})=\mathbb{Z}$.

Temos ainda:

- $\varphi(1)=(0,2,2)$;

- $\quad-\theta(1,0,0)=(-1,1)$;

$-\theta(0,1,0)=(-1,1)$;

- $\theta(0,0,1)=(1,-1)$;

Portanto: 
- $H^{0}(K ; \widetilde{\mathbb{Z}})=0$;

- $H^{1}(K ; \widetilde{\mathbb{Z}})=\mathbb{Z} \oplus \mathbb{Z}_{2}$;

- $H^{2}(K ; \widetilde{\mathbb{Z}})=\mathbb{Z}$.

3. $\left(\alpha^{*}=-i d\right.$ e $\left.\beta^{*}=i d\right)$ Denotado $\overline{\mathbb{Z}}$ :

Temos que:

- $\quad-\phi(1,0)=(1,-1,-1) ;$

$-\phi(0,1)=(-1,-1,1)$;

- $\quad-\psi(1,0,0)=-2$;

- $\psi(0,1,0)=0$;

$-\psi(0,0,1)=-2$;

Portanto:

- $H_{0}(K ; \overline{\mathbb{Z}})=\mathbb{Z}_{2}$

- $H_{1}(K ; \overline{\mathbb{Z}})=\mathbb{Z}_{2}$;

- $H_{2}(K ; \overline{\mathbb{Z}})=0$.

Temos ainda:

- $\varphi(1)=(2,0,2)$;

- $\quad-\theta(1,0,0)=(-1,1)$;

$-\theta(0,1,0)=(-1,1)$;

- $\theta(0,0,1)=(1,-1)$;

Portanto:

- $H^{0}(K ; \overline{\mathbb{Z}})=0$;

- $H^{1}(K ; \overline{\mathbb{Z}})=\mathbb{Z} \oplus \mathbb{Z}_{2}$;

- $H^{2}(K ; \overline{\mathbb{Z}})=\mathbb{Z}$.

4. $\left(\alpha^{*}=-i d\right.$ e $\left.\beta^{*}=-i d\right)$ Denotado $\overline{\mathbb{Z}}^{\prime}$ :

Temos que:

- $\quad-\phi(1,0)=(1,-1,-1)$;

- $\phi(0,1)=(-1,-1,1)$;

- $-\psi(1,0,0)=-2$;

$-\psi(0,1,0)=-2$; 
$-\psi(0,0,1)=0$;

Portanto:

- $H_{0}\left(K ; \overline{\mathbb{Z}}^{\prime}\right)=\mathbb{Z}_{2}$;

- $H_{1}\left(K ; \overline{\mathbb{Z}}^{\prime}\right)=\mathbb{Z}_{2}$;

- $H_{2}\left(K ; \overline{\mathbb{Z}}^{\prime}\right)=0$.

Temos ainda:

- $\varphi(1)=(2,2,0)$;

- $\quad-\theta(1,0,0)=(-1,-1)$;

$-\theta(0,1,0)=(1,1)$;

$-\theta(0,0,1)=(1,-1)$;

Portanto:

- $H^{0}\left(K ; \overline{\mathbb{Z}}^{\prime}\right)=0$;

- $H^{1}\left(K ; \overline{\mathbb{Z}}^{\prime}\right)=\mathbb{Z}_{2}$;

- $H^{2}\left(K ; \overline{\mathbb{Z}}^{\prime}\right)=\mathbb{Z}_{2}$.

Com os resultados obtidos podemos construir a seguinte tabela ${ }^{4}$ :

\begin{tabular}{|c|c|c|c|c|}
\hline S.C.L. & $\mathbb{Z}$ & $\widetilde{\mathbb{Z}}$ & $\overline{\mathbb{Z}}$ & $\overline{\mathbb{Z}}^{\prime}$ \\
\hline \hline$H_{0}$ & $\mathbb{Z}$ & $\mathbb{Z}_{2}$ & $\mathbb{Z}_{2}$ & $\mathbb{Z}_{2}$ \\
$H_{1}$ & $\mathbb{Z} \oplus \mathbb{Z}_{2}$ & $\mathbb{Z}$ & $\mathbb{Z}_{2}$ & $\mathbb{Z}_{2}$ \\
$H_{2}$ & 0 & $\mathbb{Z}$ & 0 & 0 \\
\hline \hline$H^{0}$ & $\mathbb{Z}$ & 0 & 0 & 0 \\
$H^{1}$ & $\mathbb{Z}$ & $\mathbb{Z} \oplus \mathbb{Z}_{2}$ & $\mathbb{Z} \oplus \mathbb{Z}_{2}$ & $\mathbb{Z}_{2}$ \\
$H^{2}$ & $\mathbb{Z}_{2}$ & $\mathbb{Z}$ & $\mathbb{Z}$ & $\mathbb{Z}_{2}$ \\
\hline
\end{tabular}

\section{$1.8 H_{n}(M ; \widetilde{\mathbb{Z}})$}

O objetivo dessa seção é apresentar uma demonstração direta para o fato de que com o sistema de coeficientes locais dado pela orientação de uma variedade compacta e sem bordo, o grupo de homologia em dimensão máxima é não trivial. Para tanto seguiremos o caminho desenvolvido em [Vick, Capítulo 5].

\footnotetext{
${ }^{4}$ Note que aqui estão todos os sistemas de coeficientes locais possíveis, com imagem em $\mathbb{Z}$, para a garrafa de Klein
} 
induzido da inclusão, que é um isomorfismo.

Assim tomando-se como base para uma topologia em $\Upsilon$ os conjuntos:

$$
U_{V, \theta, k}=\left\{<\left(j_{V, p *}(\theta), k\right)>\mid p \in V \text { e } k \in \mathbb{Z}\right\}
$$

onde $U$ percorre todas as bolas-próprias em $M, \theta$ percorre todas as classes em $H_{n}(M, M \backslash V ; \widetilde{\mathbb{Z}})$ e $k$ todos os inteiros temos que, fixados $V, \theta$ e $k$ a projeção natural de $U_{V, \theta, k}$ em $M$ é um homeomorfismo local.

Definimos então $s: M \rightarrow \Upsilon$ dada por:

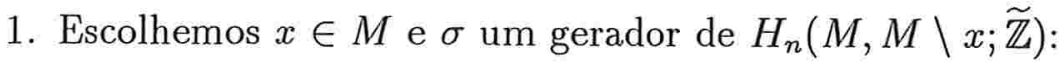

$$
s(x)=<(\sigma, 1)>\in T_{x} \subset \Upsilon .
$$

2. Dado $y \in M$ com $y \neq x$ tomamos $\alpha$ uma curva simples em $M \operatorname{com} \alpha(0)=y$ e $\alpha(1)=x$. Sendo $U \subset M$ uma bola-própria em $M$ tal que $\alpha(t) \in U$ para todo $t$ definimos:

$$
s(y)=<\left(j_{V, y *} \circ j_{V, x *}^{-1}(\sigma), \widetilde{\mathbb{Z}}(\alpha)(1)\right)>
$$

lembrando que $\widetilde{\mathbb{Z}}(\alpha): \mathbb{Z} \rightarrow \mathbb{Z}$ é o isomorfismo induzido por $\alpha$ no sistema de coeficientes locais $\widetilde{\mathbb{Z}}$.

Teorema 1.38. $s(y)$ independe de $\alpha$.

Demonstração: Sejam $\alpha$ e $\alpha^{\prime}$ duas curvas simple em $M$ com $\alpha(0)=\alpha^{\prime}(0)=y$ e $\alpha(1)=\alpha^{\prime}(1)=x$ temos que:

- $j_{V, y *} \circ j_{V, x *}^{-1}(\sigma)=j_{V^{\prime}, y *} \circ j_{V^{\prime}, x *}^{-1}(\sigma) \Leftrightarrow \operatorname{sinal}\left(\alpha \cdot \alpha^{\prime}\right)=1$.

- $j_{V, y *} \circ j_{V, x *}^{-1}(\sigma)=-\left(j_{V^{\prime}, y *} \circ j_{V^{\prime}, x *}^{-1}(\sigma)\right) \Leftrightarrow \operatorname{sinal}\left(\alpha \cdot \alpha^{\prime}\right)=-1$.

- $\widetilde{\mathbb{Z}}(\alpha)(1)=\widetilde{\mathbb{Z}}\left(\alpha^{\prime}\right)(1) \Leftrightarrow \operatorname{sinal}\left(\alpha \cdot \alpha^{\prime}\right)=1$.

- $\widetilde{\mathbb{Z}}(\alpha)(1)=-\widetilde{\mathbb{Z}}\left(\alpha^{\prime}\right)(1) \Leftrightarrow \operatorname{sinal}\left(\alpha \cdot \alpha^{\prime}\right)=-1$.

Assim

- $\widetilde{\mathbb{Z}}(\alpha)(1)=\widetilde{\mathbb{Z}}\left(\alpha^{\prime}\right)(1) \Leftrightarrow j_{V, y *} \circ j_{V, x *}^{-1}(\sigma)=j_{V^{\prime}, y *} \circ j_{V^{\prime}, x *}^{-1}(\sigma)$

- $\widetilde{\mathbb{Z}}(\alpha)(1)=-\widetilde{\mathbb{Z}}\left(\alpha^{\prime}\right)(1) \Leftrightarrow j_{V, y *} \circ j_{V, x *}^{-1}(\sigma)=-\left(j_{V^{\prime}, y *} \circ j_{V^{\prime}, x *}^{-1}(\sigma)\right)$ 
Teorema 1.35. Se $M$ é uma variedade n-dimensional, não compacta e sem bordo, então $H_{n}(M ; \widetilde{\mathbb{Z}})=0$.

Demonstração: Veja [Vick, Teorema 5.5, página 134] para o caso de coeficientes usuais, o caso com coeficientes induzidos da orientação de $M$ pode ser obtido de maneira similar.

Corolário 1.36. Se $M$ é uma variedade fechada (conexa, compacta e sem bordo) $n$-dimensional, então, para todo $p \in M$ a aplicação induzida da inclusão:

$$
i_{p *}: H_{n}(M ; \widetilde{\mathbb{Z}}) \rightarrow H_{n}(M, M \backslash p ; \widetilde{\mathbb{Z}})
$$

é injetora.

Demonstração: Seja $z \in H_{n}(M ; \widetilde{\mathbb{Z}}) \operatorname{com} i_{p *}(z)=0$. Usando a sequência do $\operatorname{par}(M, M \backslash p)$ concluímos que existe um elemento $z^{\prime} \in H_{n}(M \backslash p ; \widetilde{\mathbb{Z}})$ tal que $j\left(z^{\prime}\right)=z$ onde $j$ é a inclusão de $M \backslash p$ em $M$. Como $M \backslash p$ não é compacto, pelo Teorema $1.35 H_{n}(M \backslash p ; \widetilde{\mathbb{Z}})=0$ e assim $z^{\prime}=0$ e portanto $z=0$.

Corolário 1.37. Se $M$ é uma variedade n-dimensional, conexa e sem bordo então:

$$
H_{n}(M ; \widetilde{\mathbb{Z}})=0 \quad \text { ou } \quad H_{n}(M ; \widetilde{\mathbb{Z}})=\mathbb{Z} .
$$

Demonstração: Pelo Corolário 1.36 temos que:

$$
i_{p *}: H_{n}(M ; \widetilde{\mathbb{Z}}) \rightarrow H_{n}(M, M \backslash p ; \widetilde{\mathbb{Z}})
$$

é injetora, como $H_{n}(M, M \backslash p ; \widetilde{\mathbb{Z}})=\mathbb{Z}$ temos que $H_{n}(M ; \widetilde{\mathbb{Z}})=0$ ou $H_{n}(M ; \widetilde{\mathbb{Z}})=\mathbb{Z}$.

Para provarmos que $H_{n}(M ; \widetilde{\mathbb{Z}})=\mathbb{Z}$ basta mostrar que existe uma classe de homologia em $H_{n}(M ; \widetilde{\mathbb{Z}})$ não trivial.

Em $\mathbb{Z} \times \mathbb{Z}$ definimos Rel como a relação de equivalência dada por $(a, b) \sim$ $(-a,-b)$.

Para cada $p \in M$ definimos $T_{p}=\frac{H_{n}(M, M \backslash p ; \tilde{\mathbb{Z}}) \times \mathbb{Z}}{\operatorname{Rel}} \approx \frac{\mathbb{Z} \times \mathbb{Z}}{\operatorname{Rel}}$ e tomamos o conjunto:

$$
\Upsilon=\bigcup_{p \in M} T_{p}
$$

Para cada $V$ uma bola-própria (um aberto de $\mathrm{M}$ cujo fecho é homeomorfo ao disco $\left.D^{n}\right)^{5}$ e para cada $p \in V$, existe:

${ }^{5}$ Ver [Vick, página 138]

$$
j_{V, p *}: H_{n}(M, M \backslash V ; \widetilde{\mathbb{Z}}) \rightarrow H_{n}(M, M \backslash p ; \widetilde{\mathbb{Z}})
$$


Como $j_{V, y *} \circ j_{V, x *}^{-1}= \pm i d, \widetilde{\mathbb{Z}}(\alpha)= \pm i d, j_{V^{\prime}, y *} \circ j_{V^{\prime}, x *}^{-1}= \pm i d$ e $\widetilde{\mathbb{Z}}\left(\alpha^{\prime}\right)= \pm i d$ obtemos o resultado desejado.

Definição 1.39. s é chamada uma orientação de $M$ com respeito ao sistema de coeficientes locais $\widetilde{\mathbb{Z}}$.

Vale a pena ressaltar que a construção de $s$, no caso de $M$ ser orientável, corresponde a construção de uma orientação como em [Vick, Capítulo 5, página 138].

Lema 1.40. Sejam $M$ uma variedade fechada e $U$ um aberto de $M$, se um elemento $\theta \in H_{n}(M, U ; \widetilde{\mathbb{Z}})$ é tal que $j_{U, p *}(\theta)=0$ para todo $p \in M \backslash U$ então $\theta=0$.

Demonstração: Similar a demostração de [Vick, Lema 5.8, página 139].

Lema 1.41. Se $M$ é uma variedade fechada e $s: M \rightarrow \Upsilon$ é uma orientação de $M$ com respeito ao sistema de coeficientes locais $\widetilde{\mathbb{Z}}$ então existe uma classe $\xi \in H_{n}(M ; \widetilde{\mathbb{Z}})$ tal que $<\left(i_{p *}(\xi), 1\right)>=s(p)$ para todo $p \in M$.

Demonstração: Da definição de $s$ temos que dado $p \in M$ existe uma bolaprópria $V_{p}$ contendo $p$ e um elemento $\sigma_{p} \in H_{n}\left(M, M \backslash \overline{V_{p}} ; \widetilde{\mathbb{Z}}\right)$ tal que:

$$
<\left(j_{V_{p}, q *}\left(\sigma_{p}\right), 1\right)>=s(q)
$$

para todo $q \in V_{p}$.

Como $M$ é compacta existe uma coleção finita $\left\{V_{1}, V_{2}, \cdots, V_{k}\right\}$ de bolas-próprias como as acima $\left(p_{i} \in V_{i}\right.$ e $\left.\sigma_{p_{i}}=\sigma_{i}\right)$ cobrindo $M$. Faremos uma indução sobre esse número. que

Suponha que exista um elemento $\xi_{m} \in H_{n}\left(M, M \backslash\left(\overline{V_{1}} \cup \overline{V_{2}} \cup \cdots \cup \overline{V_{m}}\right) ; \widetilde{\mathbb{Z}}\right)$ tal

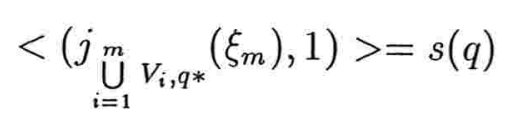

para todo $q \in \bigcup_{i=1}^{m} V_{i}$.

Tomando-se a sequência de Mayer-Vietoris relativa: 


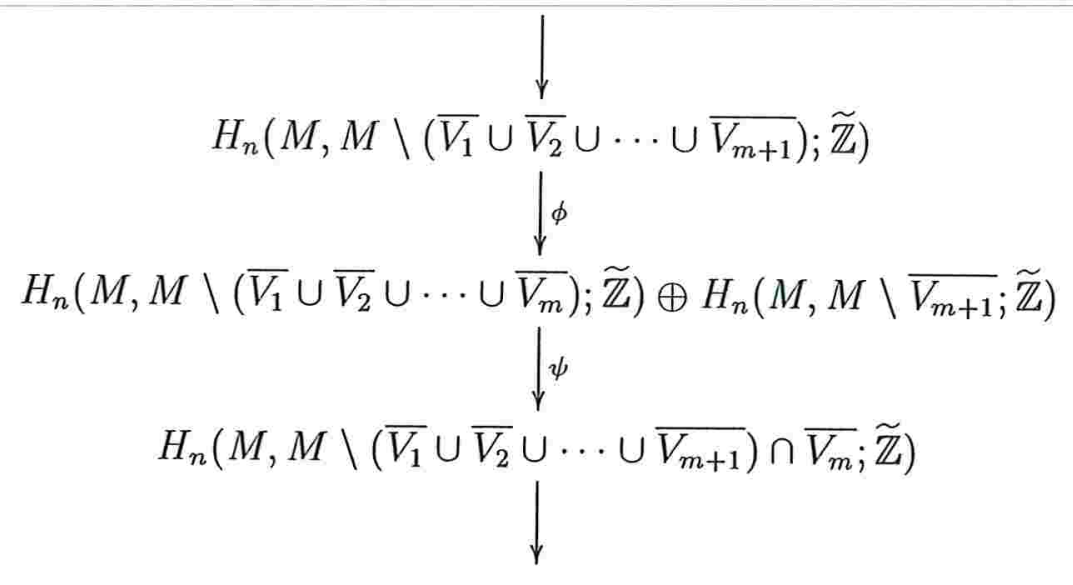

Tomando-se $\left(\xi_{m},-\sigma_{m+1}\right) \in H_{n}\left(M, M \backslash\left(\overline{V_{1}} \cup \overline{V_{2}} \cup \cdots \cup \overline{V_{m}}\right) ; \widetilde{\mathbb{Z}}\right) \oplus H_{n}(M, M \backslash$ $\left.\overline{V_{m+1}} ; \widetilde{\mathbb{Z}}\right)$ e considerando:

$$
\zeta=\psi\left(\xi_{m},-\sigma_{m+1}\right) \in H_{n}\left(M, M \backslash\left(\overline{V_{1}} \cup \overline{V_{2}} \cup \cdots \cup \overline{V_{m+1}}\right) \cap \overline{V_{m}} ; \widetilde{\mathbb{Z}}\right)
$$

temos que $j_{\left(\overline{V_{1}} \cup \overline{V_{2}} \cup \cdots \cup \overline{V_{m+1}}\right) \cap \overline{V_{m}}, q *}(\zeta)=0$ para todo $q \in \overline{V_{1}} \cup \overline{V_{2}} \cup \cdots \cup \overline{V_{m+1}} \cap \overline{V_{m}}$.

Pelo Lema $1.40, \zeta=0$. Como a sequência acima é exata existe:

$$
\xi_{m+1} \in H_{n}\left(M, M \backslash\left(\overline{V_{1}} \cup \overline{V_{2}} \cup \cdots \cup \overline{V_{m+1}}\right) ; \widetilde{\mathbb{Z}}\right)
$$

$\operatorname{com} \phi\left(\xi_{m+1}\right)=\left(\xi_{m},-\sigma_{m+1}\right)$.

Observamos agora que:

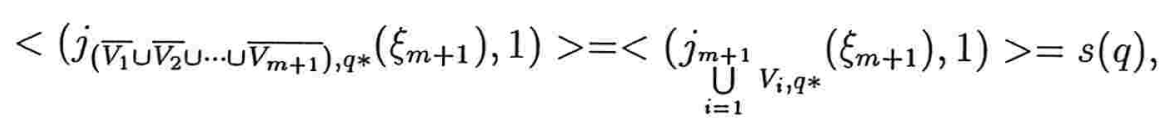

o que completa a indução.

Teorema 1.42. Se $M$ é uma variedade $n$-dimensional fechada então $H_{n}(M ; \widetilde{\mathbb{Z}})=$ $\mathbb{Z}$.

Demonstração: Pelo Corolário $1.37 H_{n}(M ; \widetilde{\mathbb{Z}})=0$ ou $H_{n}(M ; \widetilde{\mathbb{Z}})=\mathbb{Z}$, pelo Lema 1.41 existe uma classe $\xi$ em $H_{n}(M ; \widetilde{\mathbb{Z}})$, tal que $\left\langle\left(i_{p *}(\xi), 1\right)\right\rangle=s(p)$.

Como $s(p)=(<\sigma, 1\rangle)$ com $\sigma$ um gerador de $H_{n}(M, M \backslash p ; \widetilde{\mathbb{Z}})=\mathbb{Z}$ a classe $\xi$ é não trivial portanto $H_{n}(M ; \widetilde{\mathbb{Z}})=\mathbb{Z}$. 


\section{Preliminares}

O objetivo desse capítulo é apresentar algumas definições, propriedades e resultados relativos à grau, índice de coincidência e número de Lefschetz para aplicações entre variedades não necessariamente orientáveis que nos serão úteis posteriormente. Assumiremos que o leitor tem alguma familiaridade com a teoria de coincidência de Nielsen e com a geometria das superfícies.

\subsection{Grau de Aplicações}

Como vamos tratar de superfícies não necessariamente orientáveis usaremos a definição de grau de uma aplicação entre duas variedades quaisquer apresentada em [Epstein].

Dadas $M$ e $N$ duas variedades compactas sem bordo e $f: M \mapsto N$ podemos construir o seguinte diagrama comutativo:

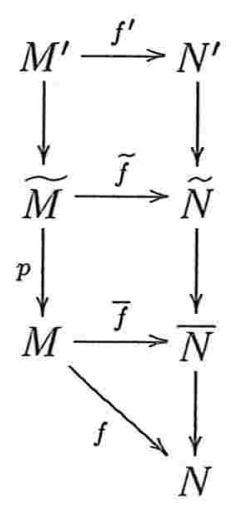

Onde: 
- $p: \widetilde{M} \mapsto M$ é o recobrimento duplo orientável de $M$ se $M$ é não orientável ou é a identidade se $M$ é-orientável;

- $\bar{N}$ é o recobrimento de $N$ associado a $f_{\#}\left(\pi_{1}(M)\right) \subseteq \pi_{1}(N)$;

- $\tilde{N}$ é o recobrimento de $N$ associado a $(f \circ p)_{\#}\left(\pi_{1}(\widetilde{M})\right)$;

- $N^{\prime}$ é o recobrimento duplo orientável de $\widetilde{N}$ se $\widetilde{N}$ for não orientável ou $N^{\prime}=\widetilde{N}$ se $\widetilde{N}$ for orientável;

- $M^{\prime}$ é o recobrimento induzido por $\widetilde{f}$.

Definição 2.1. $f: M \mapsto N$ é do tipo 1 se uma das seguintes condições é satisfeita:

1. $M$ e $\bar{N}$ são orientáveis;

2. $M$ e $\bar{N}$ são não-orientáveis e $\tilde{N}$ é orientável;

Definição 2.2. $f: M \mapsto N$ é do tipo 2 se uma das seguintes condições é satisfeita:

1. $M$ é não-orientável, $\widetilde{N} \neq \bar{N}$ e $\widetilde{N}$ é não-orientável;

2. $M$ é orientável e $\bar{N}$ é não-orientável;

3. $M$ é não-orientável, $\widetilde{N} \neq \bar{N}$ e $\widetilde{N}$ é orientável;

Definição 2.3. $f: M \mapsto N$ é do tipo 3 se $M$ é não-orientável e $\widetilde{N}=\bar{N}$.

Denotando por $\operatorname{deg}(f)$ e $\operatorname{deg}\left(f, \mathbb{Z}_{2}\right)$ respectivamente o grau usual de uma aplicação $f$ entre variedades orientáveis e o grau $\bmod 2$ definido com coeficientes em $\mathbb{Z}_{2}$ para uma $f$ entre variedades quaisquer e tomando-se $j=\left|\pi_{1}(N): f_{\#}\left(\pi_{1}(M)\right)\right|$ (que corresponde ao número de folhas do recobrimento $\bar{N} \mapsto N$ ) podemos então definir $^{1}$ o grau de $f: M \mapsto N$ :

$$
\operatorname{deg}(f)=\left\{\begin{array}{cll}
0 & \text { se } \quad(j=\infty) \text { ou }(f \text { é do tipo } 2) \text { ou } \\
& \left(f \text { é do tipo } 3 \text { e } \operatorname{deg}\left(\bar{f}, \mathbb{Z}_{2}\right)=0\right) ; \\
j|\operatorname{deg}(\tilde{f})| & \text { se } f \text { é do tipo } 1 ; \\
j & \text { se } f \text { é do tipo } 3 \text { e } \operatorname{deg}\left(\bar{f}, \mathbb{Z}_{2}\right) \neq 0 .
\end{array}\right.
$$

Note que se $M$ e $N$ são orientáveis a definição corresponde a usual.

\footnotetext{
${ }^{1}$ Para maiores detalhes ver [Epstein]
} 


\subsection{Aplicações Fiéis à Orientação}

Dada $M$ uma variedade $n$-dimensional sem bordo denotaremos por $\widetilde{\mathbb{Z}}$ o sistema de coeficientes locais dado pela orientação de $M$ com valores em $\mathbb{Z}$, ou seja $G(x)=$ $\mathbb{Z}$ para todo $x \in M$ e $G(\alpha): \mathbb{Z} \mapsto \mathbb{Z}$ é tal que $G(\alpha)(1)=(-1)^{\operatorname{sinal}(\alpha)}$ para todo $\alpha \in \pi_{1}(M)$ (ver definição 1.12). Analogamente usaremos a notação $\widetilde{\mathbb{Q}}$ quando $G(x)=\mathbb{Q}$.

Definição 2.4. Dadas duas variedades $X$ e $Y$ uma aplicação $f: X \mapsto Y$ é fiel à orientação $o^{2}$ se: $\forall \alpha \in \pi_{1}(X)$ vale que sinal $(\alpha) \cdot \operatorname{sinal}(f(\alpha))=1$.

Teorema 2.5. Se $f$ é fiel a orientação então $f$ induz um homomorfismo entre os grupos de homologia com coeficientes dados pelas orientações de $X$ e de $Y$.

Demonstração: Basta observar que como $f$ é fiel a orientação o sistema de coeficientes locais $\widetilde{\mathbb{Z}}$ em $X$ é isomorfo ao sistema $f^{*}(\widetilde{\mathbb{Z}})$ induzido por $f$.

\subsection{Classes de Coincidências}

Apesar de lidarmos apenas com superfícies, apresentaremos algumas definições e propriedades básicas das classes de coincidências no contexto geral de complexos simpliciais.

Definição 2.6. Uma coincidência entre duas funções $f_{1}, f_{2}: X \mapsto Y$ é um ponto $x \in X$ tal que $f_{1}(x)=f_{2}(x)$.

Chamaremos de $\operatorname{Coin}\left(f_{1}, f_{2}\right)$ o conjunto das coincidências do par $f_{1}, f_{2}$.

Sejam $X$ e $Y$ são poliedros conexos, $X$ compacto, $p: \tilde{X} \rightarrow X$ e $q: \tilde{Y} \rightarrow Y$ seus recobrimentos universais. Denotando por $\mathfrak{D}(X)$ o grupo das aplicações $\gamma: \widetilde{X} \rightarrow \widetilde{X}$ tais que $\gamma \circ p=p \circ i d$, temos que valem as seguintes proposições:

Proposição 2.7. Se $\tilde{f}: \widetilde{X} \rightarrow \widetilde{Y}$ é um levantamento fixado de f existe $\widetilde{f}_{\pi}$ : $\mathfrak{D}(X) \rightarrow \mathfrak{D}(Y)$ endomorfismo tal que:

$$
\tilde{f}_{\pi}(\gamma) \circ \tilde{f}=\tilde{f} \circ \gamma .
$$

Demonstração: [Jiang 1, página 25].

\footnotetext{
${ }^{2}$ Em inglês: orientation true
} 
Proposição 2.8. Se $\widetilde{f}_{1}$ e ${\widetilde{f_{1}}}_{1}^{\prime}$ são levantamentos de $f_{1}: X \rightarrow Y$ e $\tilde{f}_{2}$ e $\tilde{f}_{2}^{\prime}$ são levantamentos de $f_{2}: X \rightarrow Y$ então $p\left(\operatorname{Coin}\left(\tilde{f}_{1}, \tilde{f}_{2}\right)\right)=p\left(\operatorname{Coin}\left(\tilde{f}_{1}^{\prime}, \tilde{f}_{2}^{\prime}\right)\right)$ se, e somente se, existirem $\gamma \in \mathfrak{D}(X)$ e $\alpha \in \mathfrak{D}(Y)$ tais que:

$$
\tilde{f}_{1}^{\prime}=\alpha \circ \tilde{f}_{1} \text { e } \tilde{f}_{2}^{\prime}=\alpha \circ \widetilde{f}_{2 \pi}(\gamma) \circ \tilde{f}_{2} \circ \gamma^{-1} .
$$

Demonstração: [Oliveira, página 25].

Podemos então definir:

Definição 2.9. Sejam $\widetilde{f}_{1}$ e $\widetilde{f}_{1}^{\prime}$ levantamentos de $f_{1} ; \widetilde{f}_{2}$ e $\widetilde{f}_{2}^{\prime}$ levantamentos de $f_{2}$. $O \operatorname{par}\left(\widetilde{f}_{1}, \widetilde{f}_{2}\right)$ é equivalente $\dot{a}\left(\widetilde{f}_{1}^{\prime}, \widetilde{f}_{2}^{\prime}\right)$ se existem $\gamma \in \mathfrak{D}(X)$ e $\alpha \in \mathfrak{D}(Y)$ tais que:

$$
\widetilde{f}_{1}^{\prime}=\alpha \circ \widetilde{f}_{1} \text { e } \tilde{f}_{2}^{\prime}=\alpha \circ \widetilde{f}_{2 \pi}(\gamma) \circ \widetilde{f}_{2} \circ \gamma^{-1} .
$$

As classes de equivalência assim determinadas são chamadas classes de levantamento de $f_{1}$ e $f_{2}$.

Usando a proposição 2.8 podemos passar a noção de classe para as coincidências de $f_{1}$ e $f_{2}$.

Definição 2.10. O cojunto $p\left(\operatorname{Coin}\left(\tilde{f}_{1}, \widetilde{f}_{2}\right)\right) \subset \operatorname{Coin}\left(f_{1}, f_{2}\right)$, determinado pela classe $\left[\left(\widetilde{f}_{1}, \widetilde{f}_{2}\right)\right]$ é denominado classe de coincidência de $f_{1}$ e $f_{2}$.

Corolário 2.11. Coin $\left(f_{1}, f_{2}\right)$ é a união disjunta de classes de coincidência.

Corolário 2.12. Dois pontos $x_{0}, x_{1} \in \operatorname{Coin}\left(f_{1}, f_{2}\right)$ estão na mesma classe de coincidência de $f_{1}, f_{2}: X \mapsto Y$ se, e somente se, existe uma curva $\zeta$ de $x_{0}$ até $x_{1}$ em $X$ tal que $f_{1} \circ \zeta \simeq f_{2} \circ \zeta \operatorname{rel}\{0,1\}$.

Demonstração: [Oliveira, página 30].

Corolário 2.13. Se $\mathbb{F} \subset$ Coin $\left(f_{1}, f_{2}\right)$ é uma classe de coincidência então $\mathbb{F}$ é um aberto em $\operatorname{Coin}\left(f_{1}, f_{2}\right)$.

Demonstração: [Oliveira, página 30].

Corolário 2.14. Coin $\left(f_{1}, f_{2}\right)$ contém somente um número finito de classes.

Demonstração: [Oliveira, página 30].

Corolário 2.15. Se $\mathbb{F}$ é uma classe de coincidência de $f_{1}, f_{2}$ então $\mathbb{F}$ é um compacto em $X$.

Demonstração: [Oliveira, página 31].

Teorema 2.16. Sejam $f_{1}, f_{1}^{\prime}, f_{2}, f_{2}^{\prime}: X \rightarrow Y, X$ e $Y$ poliedros conexos, $X$ compacto, $f_{1} \simeq f_{1}^{\prime}$ e $f_{2} \simeq f_{2}^{\prime}$ então as classes de coincidência de $f_{1}, f_{2}$ estão em correspondência biunívoca com as de $f_{1}^{\prime}, f_{2}^{\prime}$.

Demonstração: [Oliveira, página 31]. 


\section{4 Índice e Número de Lefschtez}

A situação apresentada aqui consiste de um caso particular dos resultados de [Gonçalves \& Jezierski] pois lidamos com variedades sem bordo e nossos sistemas de coeficientes sempre têm valores em $\mathbb{Z}$ ou $\mathbb{Q}$. Assim para uma exposição detalhada consulte [Gonçalves \& Jezierski].

Denotando por $z_{M, x}$ o gerador canônico de $H_{n}(M, M \backslash x ; \widetilde{\mathbb{Z}}) \approx \mathbb{Z}$ temos:

Lema 2.17 (Classe Fundamental). Para todo conjunto compacto $C \subset M$ existe um único elemento $z_{M, C} \in H_{n}(M, M \backslash C ; \widetilde{\mathbb{Z}})$ tal que para todo $x \in C$ sua imagem pelo homomorfismo natural $H_{n}(M, M \backslash C ; \widetilde{\mathbb{Z}}) \mapsto H_{n}(M, M \backslash x ; \widetilde{\mathbb{Z}})$ é $z_{M, x}$.

Demonstração: [Gonçalves \& Jezierski, Lema 3.1, página 5].

Observação 2.18. Note que se $M$ é compacta temos que $z_{M, M}$ é um gerador de $H_{n}(M, \widetilde{\mathbb{Z}})$.

Lema 2.19 (Dualidade de Poincaré). Se $M$ é uma n-variedade compacta $e$ sem bordo então o produto cap:

$$
\cap z_{M}: H^{p}(M ; \mathbb{Z}) \mapsto H_{n-p}(M ; \widetilde{\mathbb{Z}})
$$

denotado $D_{M}$ é um isomorfismo.

Demonstração: [Gonçalves \& Jezierski, Corolário 3.3, página 6].

Definindo $j_{x}: M \times M \mapsto M$ tal que $j_{x}(y)=(x, y) \forall x, y \in M$ e denotando por $\triangle$ a diagonal em $M \times M$ temos:

Lema 2.20 (Classe de Thom). Se $M$ é uma n-variedade sem bordo existe um único elemento $U_{M} \in H^{n}(M \times M, M \times M \backslash \Delta ;(\mathbb{Z} \times \widetilde{\mathbb{Z}}))$ cuja restrição a qualquer fibra é o dual da classe fundamental, ou seja $\left\langle\left(j_{x}\right)^{*}\left(U_{M}\right), z_{M, x}\right\rangle=1$

Demonstração: [Gonçalves \& Jezierski, Lema 3.4, página 6] e [Spanier, 4.7].

Tomando-se $\widetilde{U}_{M} \in H^{n}(M \times M)$ a restrição da classe de Thom e denotando por $d$ a inclusão $d(x)=(x, x) \in M \times M$ podemos definir:

Definição 2.21. Dadas $M, N$ n-variedades compactas e sem bordo e $f_{1}, f_{2}: M \mapsto$ $N$ aplicaçôes com $f_{2}$ fiel à orientação, o índice do par $\left(f_{1}, f_{2}\right)$ (ind $\left(f_{1}, f_{2}\right)$ ) é a imagem da classe fundamental $z_{M}$ pela sequência de homomorfismos:

$$
H_{n}(M ; \widetilde{\mathbb{Z}}) \stackrel{d_{*}}{\longrightarrow} H_{n}(M \times M ; \mathbb{Z} \times \widetilde{\mathbb{Z}}) \stackrel{\left(f_{1} \times f_{2}\right)_{*}}{\longrightarrow} H_{n}(N \times N ; \mathbb{Z} \times \widetilde{\mathbb{Z}}) \stackrel{<\widetilde{U}_{N} \cdot>}{\longrightarrow} \mathbb{Z} .
$$

Ou seja ind $\left(f_{1}, f_{2}\right)=<\widetilde{U}_{N},\left(f_{1} \times f_{2}\right)_{*} d_{*}\left(z_{M}\right)>$. 
Nas condições acima temos $f_{2}$ fiel à orientação, assim $f_{2}$ induz homomorfismos $f_{2}^{*}: H_{q}(N, \widetilde{\mathbb{Q}}) \mapsto H_{q}(M, \widetilde{\mathbb{Q}})$, usando o Lema 2.19 podemos construir o seguinte diagrama comutativo:

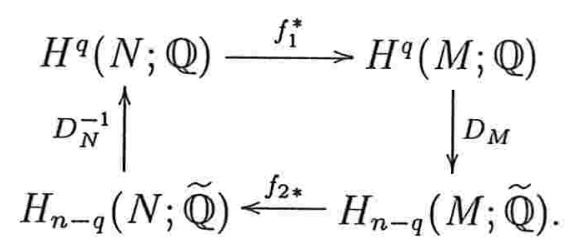

Definição 2.22 (Número de Lefschetz). Dadas $M, N$-variedades compactas e sem bordo e $f_{1}, f_{2}: M \mapsto N$ aplicações com $f_{2}$ fiel à orientação tomamos:

$$
L\left(f_{1}, f_{2}\right)=\sum_{q=0}^{n}(-1)^{n} \operatorname{tr}\left(\theta_{q}\right)
$$

onde $\theta_{q}: H^{q}(N ; \mathbb{Q}) \mapsto H^{q}(M ; \mathbb{Q})$ é descrita como a composta $D_{N}^{-1} \circ f_{2 *} \circ D_{M} \circ f_{1}^{*}$.

Claramente $L\left(f_{1}, f_{2}\right)$ é um invariante homotópico.

Teorema 2.23 (Normalização). Nas condições da definição acima:

$$
L\left(f_{1}, f_{2}\right)=\operatorname{ind}\left(f_{1}, f_{2}\right) .
$$

Demonstração: [Gonçalves \& Jezierski, Teorema 4.1,página 10].

Damos agora uma definição de índice "local" de coincidência do par $\left(f_{1}, f_{2}\right)$.

Definição 2.24. Dadas $M, N$ n-variedades compactas e sem bordo e $f_{1}, f_{2}: M \mapsto$ $N$ aplicações com $f_{2}$ fiel à orientação, se $C \subset C \operatorname{Coin}\left(f_{1}, f_{2}\right)$ é aberto e fechado em Coin $\left(f_{1}, f_{2}\right)$ definimos o índice do par $\left(f_{1}, f_{2}\right)$ em $C\left(\operatorname{ind}\left(f_{1}, f_{2}, C\right)\right)$ como a imagem de $z_{M, C}$ pela composição:

$$
H_{n}(M, M \backslash C ; \widetilde{\mathbb{Z}}) \stackrel{\left(f_{1}, f_{2}\right)}{\longrightarrow} H_{n}(N \times N, N \times N \backslash \Delta ; \mathbb{Z} \times \widetilde{\mathbb{Z}}) \stackrel{<U_{N,}>}{\longrightarrow} \mathbb{Z} .
$$

ou seja ind $\left(f_{1}, f_{2}, C\right)=<U_{N},(f, g)_{*}\left(z_{M, C}\right)>$.

Note que se $C \subset U \subset M, \operatorname{com} U$ um aberto temos $H_{n}(M, M \backslash C ; \widetilde{\mathbb{Z}}) \approx H_{n}(U, U \backslash$ $C ; \widetilde{\mathbb{Z}})$, assim o índice do par $\left(f_{1}, f_{2}\right)$ pode ser calculado localmente. 


\subsection{Número de Lefschetz em Superfícies}

Usaremos o termo superfície para uma variedade compacta, sem bordo de dimensão 2, orientável ou não. Denotaremos por $S_{g}$ a superfície orientável de genus $g$ (a soma conexa de $g$ toros), por $K$ a garrafa de Klein, por $K_{g}$ a superfície não orientável obtida da soma conexa de $g$ toros com uma garrafa de Klein, por $P$ o plano projetivo real e por $P_{g}$ a superfície não orientável obtida pela soma conexa de $g$ toros e um plano projetivo.

Vale ressaltar que $K_{g}$ pode ser descrita como a soma conexa de $2 g+2$ planos projetivos e da mesma forma $P_{g}$ pode ser construída como a soma conexa de $2 g+1$ planos projetivos. O grupo fundamental de tais supefícies pode ser descrito por geradores e relações como:

$$
\begin{aligned}
& \text { - } \pi_{1}\left(S_{g}\right)=<a_{1}, b_{1}, \cdots, a_{g}, b_{g} \mid \prod_{i=1}^{g}\left[a_{i}, b_{i}\right]> \\
& \text { - } \pi_{1}\left(P_{g}\right)=<a_{1}, b_{1}, \cdots, a_{g}, b_{g}, \gamma\left|\prod_{i=1}^{g}\left[a_{i}, b_{i}\right] \gamma^{2}>\approx<\gamma_{1}, \cdots, \gamma_{2 g+1}\right| \prod_{i=1}^{2 g+1} \gamma_{i}^{2}> \\
& \text { - } \pi_{1}\left(K_{g}\right)=<a_{1}, b_{1}, \cdots, a_{g}, b_{g}, \alpha, \beta\left|\prod_{i=1}^{g}\left[a_{i}, b_{i}\right] \alpha \beta \alpha \beta^{-1}>\approx<\gamma_{1}, \cdots, \gamma_{2 g+2}\right| \prod_{i=1}^{2 g+2} \gamma_{i}^{2}>
\end{aligned}
$$

Note as representações acima são induzidas de como "construímos" tais superfícies, assim, $\gamma_{i}$ é sempre uma curva que reverte orientação, $\alpha$ e $\beta$ podem ser entendidas como as curvas canônicas da garrafa de Klein com $\beta$ revertendo a orientação local.

Os resultados apresentados podem ser encontrados, em grande parte, em [Gonçalves \& Oliveira], assim adotamos, quase sempre, as mesmas notações e definições desta referência.

Lema 2.25. Os grupos de cohomologia das superfícies ${ }^{3}$ são dados por:

$$
\begin{aligned}
& S_{g} \quad \cdot H^{0}\left(S_{g} ; \mathbb{Q}\right) \approx H^{0}\left(S_{g} ; \widetilde{\mathbb{Q}}\right)=\mathbb{Q} ; \\
& \text { - } H^{1}\left(S_{g} ; \mathbb{Q}\right) \approx H^{1}\left(S_{g} ; \widetilde{\mathbb{Q}}\right)=\underbrace{\mathbb{Q} \oplus \cdots \mathbb{Q} ;}_{2 g \text { vezes }} \\
& \text { - } H^{2}\left(S_{g} ; \mathbb{Q}\right) \approx H^{2}\left(S_{g} ; \widetilde{\mathbb{Q}}\right)=\mathbb{Q} ;
\end{aligned}
$$

\footnotetext{
${ }^{3}$ Note que no caso das superfícies orientáveis o sistema local induzido pela orientação é trivial, ou seja, coincide com o de coeficientes usuais (ver a observação 1.13 na página 5).
} 


$$
\begin{aligned}
K_{g} \quad H^{0}\left(K_{g} ; \mathbb{Q}\right)=\underbrace{\mathbb{Q}}_{2 g+1 \text { vezes }} & \\
\text { - } H^{1}\left(K_{g} ; \mathbb{Q}\right) & =\underbrace{\mathbb{Q} \oplus \cdots \mathbb{Q}} ; \\
\text { - } H^{2}\left(K_{g} ; \mathbb{Q}\right) & =0 ; \\
\text { - } H^{1}\left(K_{g} ; \widetilde{\mathbb{Q}}\right) & =0 ; \\
\text { - } H^{2}\left(K_{g} ; \widetilde{\mathbb{Q}}\right) & =\underbrace{\mathbb{Q} \oplus \cdots \mathbb{Q} ;}_{2 g+1 \text { vezes }} ; \\
\text { - } H^{0}\left(P_{g} ; \mathbb{Q}\right) & =\mathbb{Q} ; \\
\text { - } H^{1}\left(P_{g} ; \mathbb{Q}\right) & =\underbrace{\mathbb{Q} \oplus \cdots \mathbb{Q}}_{2 g \text { vezes }} ; \\
\text { - } H^{2}\left(P_{g} ;(\mathbb{Q})\right. & =0 ; \\
\text { - } H^{0}\left(P_{g} ; \widetilde{\mathbb{Q}}\right) & =0 ; \\
\text { - } H^{1}\left(P_{g} ; \widetilde{\mathbb{Q}}\right) & =\underbrace{\mathbb{Q} \oplus \cdots \mathbb{Q}}_{2 g \text { vezes }} ; \\
\text { - } H^{2}\left(P_{g} ; \widetilde{\mathbb{Q}}\right) & =\mathbb{Q} ;
\end{aligned}
$$

Demonstração: $\mathrm{O}$ caso $S_{g}$ é conhecido. Para $K_{g}$ e $P_{g}$ o cálculo pode ser feito de maneira direta (ver [Gonçalves \& Oliveira, Proposições 2.1 e 2.2, página 160]).

Tomando-se $\left\{a_{1}^{*}, b_{1}^{*}, \cdots, a_{g}^{*}, b_{g}^{*}\right\}$ a base usual de $H^{1}\left(P_{g} ; \mathbb{Q}\right)$. Seja $\left\{\widetilde{a}_{1}, \widetilde{b}_{1}, \cdots, \widetilde{a}_{g}, \widetilde{b}_{g}\right\}$ a base de $H^{1}\left(P_{g} ; \widetilde{\mathbb{Q}}\right)$ que é dual no sentido da dualidade de Poincaré (Lema 2.19) de $\left\{b_{1}^{*},-a_{1}^{*}, \cdots, b_{g}^{*},-a_{g}^{*}\right\}$. Denotando por $\alpha^{*}$ e $\beta^{*}$ os geradores provenientes de $\alpha$ e $\beta$ da descrição do $\pi_{1}$ de $K_{g}$ feita no início da seção, construímos de maneira análoga, $\left\{\widetilde{a}_{1}, \widetilde{b}_{1}, \cdots, \widetilde{a}_{g}, \widetilde{b}_{g}, \widetilde{\alpha}\right\}$ uma base de $H^{1}\left(K_{g} ; \widetilde{\mathbb{Q}}\right)$ que é dual de $\left\{b_{1}^{*},-a_{1}^{*}, \cdots, b_{g}^{*},-a_{g}^{*}, \beta^{*}\right\}$.

Nessas condições temos:

Teorema 2.26. Dadas $f_{1}, f_{2}: K_{g} \mapsto K$ com $f_{2}$ fiel à orientação e tais que:

$$
\begin{aligned}
& -f_{2}^{*}(\widetilde{\alpha})=\sum_{i=1}^{g}\left(\lambda_{i} \tilde{a}_{i}+\xi_{i} \widetilde{b}_{i}\right)+\lambda \widetilde{\alpha} \\
& \text { - } f_{2}^{*}\left(\beta^{*}\right)=\sum_{i=1}^{g}\left(m_{i} a_{i}^{*}+n_{i} b_{i}^{*}\right)+m \beta^{*}
\end{aligned}
$$


- $f_{1}^{*}\left(\beta^{*}\right)=\sum_{i=1}^{g}\left(r_{i} a_{i}^{*}+s_{i} b_{i}^{*}\right)+r \beta^{*}$

Podemos concluir que:

$$
L\left(f_{1}, f_{2}\right)=\sum_{i=1}^{g}\left(\operatorname{det}\left(\begin{array}{cc}
\lambda_{i} & \xi_{i} \\
m_{i} & n_{i}
\end{array}\right)-\operatorname{det}\left(\begin{array}{cc}
\lambda_{i} & \xi_{i} \\
r_{i} & s_{i}
\end{array}\right)\right)+\lambda(m-r)
$$

Demonstração: [Gonçalves \& Oliveira, Teorema 2.5, página 163].

Para simplificar a notação nos próximos casos definiremos algumas funções sobre matrizes $(2 h \times 2 g)$.

Se $A$ é uma matriz de ordem $(2 h \times 2 g)$ então:

$$
A_{2 m-1,2 m}^{2 l-1,2 l}=\left(\begin{array}{cc}
a_{2 m-1,2 l-1} & a_{2 m-1,2 l} \\
a_{2 m, 2 l-1} & a_{2 m, 2 l}
\end{array}\right) .
$$

Se $A$ e $B$ são matrizes $(2 h \times 2 g)$ então:

1. $\varphi(A, B)=\sum_{i=1}^{g} \sum_{j=1}^{h}\left(\operatorname{det}\left(A_{2 j-1,2 j}^{2 i-1,2 j}-B_{2 j-1,2 j}^{2 i-1,2 j}\right)-\operatorname{det}\left(B_{2 j-1,2 j}^{2 i-1,2 j}\right)-\operatorname{det}\left(A_{2 j-1,2 j}^{2 i-1,2 j}\right)\right)$;

2. $\psi(A)=\sum_{j=1}^{h}\left(A_{2 j-1,2 j}^{1,2}\right)$.

Teorema 2.27. Dadas $f_{1}, f_{2}: K_{h} \mapsto K_{g}$ com $f_{2}$ fiel à orientação e tais que:

$$
\begin{aligned}
& f_{2}^{*}(\cdot, \widetilde{\mathbb{Q}})=\left(\begin{array}{ccc} 
& & \widetilde{\xi}_{1} \\
& M & \vdots \\
& & \widetilde{\xi}_{2 h} \\
\widetilde{\lambda}_{1} & \cdots & \widetilde{\lambda}_{2 g+1}
\end{array}\right) ; \quad f_{2}^{*}(\cdot, \mathbb{Q})=\left(\begin{array}{ccc} 
& & \xi_{1} \\
& M & \vdots \\
& & \xi_{2 h} \\
\lambda_{1} & \cdots & \lambda_{2 g+1}
\end{array}\right) ; \\
& f_{1}^{*}(\cdot, \mathbb{Q})=\left(\begin{array}{ccc} 
& & \beta_{1} \\
& N & \vdots \\
& & \beta_{2 h} \\
\alpha_{1} & \cdots & \alpha_{2 g+1}
\end{array}\right)
\end{aligned}
$$


Podemos concluir que:

$$
\begin{aligned}
L\left(f_{1}, f_{2}\right)= & \varphi(N, M)+\psi(M)-\sum_{i=1}^{g}\left(\widetilde{\lambda}_{2 i-1} \alpha_{2 i}-\widetilde{\lambda}_{2 i} \alpha_{2 i-1}\right) \\
& +\sum_{j=1}^{h}\left(\widetilde{\xi}_{2 j-1} \beta_{2 j}-\widetilde{\xi}_{2 j} \beta_{2 j-1}\right)-\widetilde{\lambda}_{2 g+1} \alpha_{2 g+1}+\widetilde{\lambda}_{1} \lambda_{2} .
\end{aligned}
$$

Demonstração: [Gonçalves \& Oliveira, Teorema 2.8, página 165].

Teorema 2.28. Dadas $f_{1}, f_{2}: K_{h} \mapsto P_{g}$ com $f_{2}$ fiel à orientação e tais que:

$$
\begin{gathered}
f_{2}^{*}(\cdot, \widetilde{\mathbb{Q}})=\left(\begin{array}{ccc}
M & \\
& & \\
\widetilde{\lambda}_{1} & \cdots & \widetilde{\lambda}_{2 g}
\end{array}\right) ; \quad f_{2}^{*}(\cdot, \mathbb{Q})=\left(\begin{array}{ccc} 
& M & \\
& & \\
\lambda_{1} & \cdots & \lambda_{2 g}
\end{array}\right) ; \\
f_{1}^{*}(\cdot, \mathbb{Q})=\left(\begin{array}{ccc}
N & \\
& & \\
\alpha_{1} & \cdots & \alpha_{2 g}
\end{array}\right) ;
\end{gathered}
$$

Podemos concluir que:

$$
L\left(f_{1}, f_{2}\right)=\varphi(N, M)+\psi(M)-\sum_{i=1}^{g}\left(\widetilde{\lambda}_{2 i-1} \alpha_{2 i}-\widetilde{\lambda}_{2 i} \alpha_{2 i-1}\right)+\widetilde{\lambda}_{1} \lambda_{2}
$$

Demonstração: [Gonçalves \& Oliveira, Teorema 2.7, página 164]. 


\section{O caso orientável}

Em [Gonçalves \& Jiang] encontramos vários resultados sobre limitações de valores para índices de classes de coincidência em superfícies orientáveiss. Particularmente foram estudadas aplicações $f_{1}, f_{2}: S_{h} \mapsto S_{g}$ com $h \geq g$ e onde $S_{i}$ é a superfície orientável de genus $i$.

Usando a notação de [Gonçalves \& Jiang] definimos:

$$
B\left(g, h, d_{1}, d_{2}\right)=\sup \left\{|\operatorname{ind}(C)||| \operatorname{deg}\left(f_{1}\right)\left|=d_{1},\right| \operatorname{deg}\left(f_{2}\right) \mid=d_{2}\right\},
$$

onde o supremo é tomado para todas as classes de coincidência $C$ de todos os pares de aplicações $\left(f_{1}, f_{2}\right): S_{h} \mapsto S_{g}$ com tais graus.

Destacamos os seguintes resultados:

Teorema 3.1. $B\left(1, h, d_{1}, d_{2}\right)=\infty$ para $h>1$.[Gonçalves G Jiang, Proposição 9, página 83]

Teorema 3.2. Quando $2 \leq g \leq h$ e $0 \leq d_{1}, d_{2}<\frac{h-1}{g-1}$ (nesse caso nem $f_{1}$ nem $f_{2}$ são homotópicas a um revestimento), então: $B\left(g, h, d_{1}, d_{2}\right)=\infty$. [Gonçalves \& Jiang, Teorema 10, página 83]

Em geral o caso $d_{2}=\frac{h-1}{g-1}$ continua em aberto apesar de sob algumas condições haver uma limitação [Gonçalves \& Jiang, página 3 e Proposição 11].

Nessa seção discutiremos o caso $f_{1}, f_{2}: S_{h} \mapsto S_{g} \operatorname{com} h \leq g$.

Teorema 3.3. Se $g \geq h \geq 2$ e $d_{1}=d_{2}=0$, então $B(g, h, 0,0)=\infty$.

Observação 3.4. Esse resultado poderia ser obtido a partir dos exemplos (o par $\left.\phi_{0}, \psi_{0}\right)$ construídos na demonstração do Teorema $3.2 \mathrm{em}$ [Gonçalves 8 J Jiang], (onde o resultado para $g=h \geq 2$ já está escrito) e junto com 3.1 e 3.2 completa a demostração de: $B(g, h, 0,0)=\infty$ para qualquer par $(g, h)$ com $g \geq 1 h \geq 2$. 
Apresentamos aqui um exemplo detalhado para esse caso específico.

Demonstração: Sabemos [ZVC, página 78, Proposição 3.3.10] que toda $f$ : $S_{h} \mapsto S_{g}$ de grau zero se fatora no 1-esqueleto de $S_{g}$, além disso que toda aplicação $\tilde{f}: \bigvee_{i=1}^{2 h} S^{1} \mapsto \bigvee_{i=1}^{2 g} S^{1}$ pode ser estendida para uma $f: S_{h} \mapsto S_{g}$ se, e somente se, $\tilde{f}$ leva a relação que define o $\pi_{1}\left(S_{h}\right)$ no elemento nulo do $\pi_{1}\left(\bigvee_{i=1}^{2 g} S^{1}\right)$, ou seja $\widetilde{f}_{\#}\left(\prod_{i=1}^{h}\left[a_{2 i-1}, a_{2 i}\right]\right)=1$.

Inicialmente tomemos $h=2$ e $g=2$. Sendo $F_{4}$ o grupo livre com 4 geradores denotados por $\left\{a_{1}, a_{2}, a_{3}, a_{4}\right\}$, tomamos $\widetilde{f}_{i}: F_{4} \mapsto F_{4}$ homomorfismos tais que:

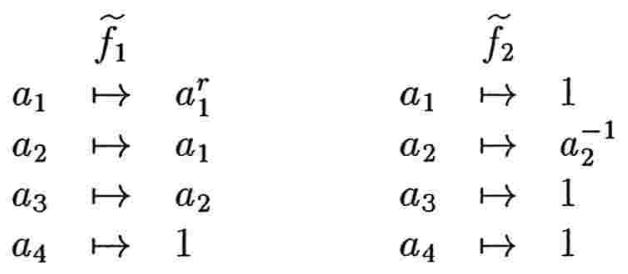

Observamos que:

$$
\widetilde{f}_{i}\left(\left[a_{1}, a_{2}\right] \cdot\left[a_{3}, a_{4}\right]\right)=1
$$

$\operatorname{assim} \tilde{f}_{i}$ admite uma extensão $f_{i}: S_{2} \mapsto S_{2}$.

Notemos que se $[w]_{R}$ é uma classe de Reidemeister associada a uma classe de Nielsen de coincidência então $w$ é uma palavra em $a_{1}$ e $a_{2}$ (pois as imagens de $\widetilde{f}_{1}$ e de $\widetilde{f}_{2}$ estão contidas no subgrupo gerado por $a_{1}$ e por $a_{2}$ ), assim $w=w^{\prime} \cdot a_{1}^{n}$ ou $w=w^{\prime} \cdot a_{2}^{n}$ e temos:

$$
\begin{gathered}
w=w^{\prime} \cdot a_{2}^{n} \sim f_{2}\left(a_{3}^{n}\right) \cdot w^{\prime} \cdot a_{2}^{n} \cdot\left(f_{1}\left(a_{3}^{n}\right)\right)^{-1} \sim w^{\prime} \cdot a_{2}^{n} \cdot a_{2}^{-n} \sim w^{\prime} . \\
w=w^{\prime} \cdot a_{1}^{n} \sim f_{2}\left(a_{2}^{n}\right) \cdot w^{\prime} \cdot a_{1}^{n} \cdot\left(f_{1}\left(a_{2}^{n}\right)\right)^{-1} \sim a_{2}^{-n} \cdot w^{\prime} \cdot a_{1}^{n} \cdot a_{1}^{-n} \sim a_{2}^{-n} \cdot w^{\prime} .
\end{gathered}
$$

Usando as relações acima um número suficiente de vezes obteremos:

$$
w \sim a_{2}^{k} \sim f_{2}\left(a_{3}^{k}\right) \cdot a_{2}^{k} \cdot\left(f_{1}\left(a_{3}^{k}\right)\right)^{-1} \sim a_{2}^{k} \cdot a_{2}^{-k} \sim 1
$$

o que nos diz que o par $\left(f_{1}, f_{2}\right)$ tem uma única clase de Nielsen de coincidência. 
Como $f_{1}^{1}$ é dada pela matriz $\left(\begin{array}{cccc}r & 1 & 0 & 0 \\ 0 & 0 & 1 & 0 \\ 0 & 0 & 0 & 0 \\ 0 & 0 & 0 & 0\end{array}\right)$ e $f_{2}^{1}$ é dada por $\left(\begin{array}{cccc}0 & 0 & 0 & 0 \\ 0 & -1 & 0 & 0 \\ 0 & 0 & 0 & 0 \\ 0 & 0 & 0 & 0\end{array}\right)$ temos que ([Gonçalves \& Jiang, Teorema 3, página 5]):

$$
L\left(f_{1}, f_{2}\right)=r
$$

Como existe uma única classe de Nilsen de coincidência temos que seu índice corresponde a $L\left(f_{1}, f_{2}\right)$ ([Vick]), assim fazendo-se $r \mapsto \infty$ concluímos que $B(2,2,0,0)=$ $\infty$.

Tomando-se, agora $f_{1}, f_{2}: F_{2 h} \mapsto F_{2 g}$ da forma:

\begin{tabular}{|c|c|c|c|c|c|}
\hline \multicolumn{3}{|c|}{$\widetilde{f_{1}}$} & \multicolumn{3}{|c|}{$\widetilde{f}_{2}$} \\
\hline$a_{1}$ & $\mapsto$ & $a_{1}^{r}$ & $a_{1}$ & $\mapsto$ & 1 \\
\hline$a_{2}$ & $\mapsto$ & $a_{1}$ & $a_{2}$ & $\mapsto$ & $a_{2}^{-1}$ \\
\hline$a_{3}$ & $\mapsto$ & $a_{2}$ & $a_{3}$ & $\mapsto$ & 1 \\
\hline$a_{4}$ & $\mapsto$ & 1 & $a_{4}$ & $\mapsto$ & 1 \\
\hline$a_{5}$ & $\mapsto$ & 1 & $a_{5}$ & $\mapsto$ & 1 \\
\hline$a_{6}$ & $\mapsto$ & 1 & $a_{6}$ & $\mapsto$ & 1 \\
\hline : & $\vdots$ & $\vdots$ & $\vdots$ & $\vdots$ & $\vdots$ \\
\hline$a_{2 h}$ & $\mapsto$ & 1 & $a_{2 h}$ & $\mapsto$ & 1 \\
\hline
\end{tabular}

Temos que:

$$
\widetilde{f}_{i}\left(\prod_{i=1}^{h}\left[a_{2 i-1}, a_{2 i}\right]\right)=1
$$

o que nos garante que tais homomorfismos admitem uma extensão $f_{i}: S_{h} \mapsto S_{g}$. Pelos mesmos argumentos usados anteriormente o par $\left(f_{1}, f_{2}\right)$ tem uma única classe de Nielsen de coincidência com índice $r$.

\subsection{Aplicações do Toro}

Sabemos [Brooks, página 125] que se $f_{1}, f_{2}: S_{1} \mapsto S_{1}$, então $|\operatorname{ind}(C)| \leq 1$ para toda classe de coincidência $C$ do par $f_{1}, f_{2}$. Vamos agora mostrar que esse resultado é verdadeiro para pares $f_{1}, f_{2}: S_{1} \mapsto S_{g}$, com $g>1$. 


\subsubsection{Um pouco da geometria de $S_{g}$}

Sabemos que o toro é uma superfície euclidiana que tem como revestimento universal o plano $\mathbb{R}^{2}$; assim fixaremos a projeção $p: \mathbb{R}^{2} \mapsto \frac{\mathbb{R}^{2}}{\mathbb{Z} \times \mathbb{Z}}$ como a aplicação de recobrimento $\mathbb{R}^{2} \mapsto S_{1}$.

A superfície $S_{g}(g>1)$ é hiperbólica e seu recobrimento universal é melhor descrito [Stillwell] como sendo o disco hiperbólico de Poincaré $\mathbb{D}^{2}$ (que é homeomorfo ao $\mathbb{R}^{2}$ ), com esse modelo podemos descrever a aplicação de recobrimento como sendo a projeção $q: \mathbb{D}^{2} \mapsto \frac{\mathbb{D}^{2}}{\pi_{1}\left(S_{g}\right)}$ onde $\pi_{1}\left(S_{g}\right)$ é visto como um reticulado em $\mathbb{D}^{2}$.

Dessa maneira temos que, fixados $y_{0} \in S_{g}$ e $\widetilde{y_{0}} \in q^{-1}\left(y_{0}\right)$, para cada $\alpha \in \pi_{1}\left(S_{g}\right)$, existe um único $y_{\alpha} \in \mathbb{D}^{2}$ com a propriedade de que o segmento de reta (hiperbólica) orientado $\widetilde{\alpha}=\overline{\widetilde{y_{0}} y_{\alpha}}$ é tal que $\langle q(\widetilde{\alpha})>=\alpha$; por [Stillwell, página 158] temos que $\widetilde{\alpha} \subset \widetilde{\alpha^{k}}$ se $k \geq 1$.

Lema 3.5. Dados $\alpha, \beta \in \pi_{1}\left(S_{g}\right)$ distintos, então existem $a, b:\{[0,1],\{0,1\}\} \mapsto$ $\left\{S_{g}, y_{0}\right\}$ tais que:

- $\langle a>=\alpha e<b>=\beta$.

- $a([0,1]) \cap b([0,1])$ é finito.

- a e b são geodésicas de $S_{g}$ (ver [Stillwell]).

onde $y_{0}$ é um ponto qualquer de $S_{g}$.

Demonstração: Se $\alpha$ e $\beta$ são não triviais tomamos $\widetilde{a}:[0,1] \mapsto \widetilde{\alpha}$ e $\widetilde{b}:[0,1] \mapsto \widetilde{\beta}$ homeomorfismos tais que $\widetilde{a}(0)=\widetilde{b}(0)=\widetilde{y_{0}}$; se $\alpha$ é trivial fazemos $a$ a aplicação constante em $y_{0}$. Tomamos então $a=q \circ \widetilde{a}$ e $b=q \circ \widetilde{b}$.

Observamos que $\mathbb{D}^{2}=\bigcup_{i \in \mathbb{N}} \overline{V_{i}}$, onde cada $V_{i}$ é uma vizinhança elementar de um elemento de $q^{-1}\left(y_{0}\right)$ com $V_{i} \cap V_{j}=\emptyset$ para $i \neq j,\left.q\right|_{\bar{V}_{i}}: \overline{V_{i}} \mapsto S_{1}$ sobrejetora e $\left.q\right|_{V_{i}}$ um homeomorfismo. Como $\widetilde{\alpha}$ e $\widetilde{\beta}$ são segmentos de reta (hiperbólicas) com início no mesmo ponto $\widetilde{y_{0}}$ e $\alpha, \beta$ são distintos $\widetilde{\alpha} \cap \widetilde{\beta}=\left\{\widetilde{y_{0}}\right\}$. Além disso $\widetilde{\alpha} \subset \bigcup_{k=1}^{m} \overline{V_{i_{k}}} \mathrm{e}$ $\widetilde{\beta} \subset \bigcup_{k^{\prime}=1}^{m} \overline{V_{i_{k^{\prime}}}}$ e para cada par $i_{k}$ e $\left.\left.i_{k^{\prime}} q\right|_{\overline{V_{i_{k}}}}(\widetilde{\alpha}) \cap q\right|_{\overline{V_{i_{k^{\prime}}}}}(\widetilde{\beta})$ tem, no máximo, um ponto temos que $a([0,1]) \cap b([0,1])$ é finito.

Segue da definição de $\widetilde{\alpha}$ e $\widetilde{\beta}$ que $a$ e $b$ são geodésicas. 


\subsubsection{Forma padrão de $f: S_{1} \mapsto S_{g}$}

Definição 3.6. Dados $w \in \pi_{1}\left(S_{g}\right)$ e $r, s \in \mathbb{Z}$ e tomando-se a superfície $S_{1}$ como o quadrado $[0,1] \times[0,1]$ com os lados identificados, ${ }_{a_{1}}^{a_{1}} x_{\substack{a_{2} \\ a_{2}}}^{a_{1}}$, definimos $f_{w, r, s}: S_{1} \mapsto S_{g}$ como:

Caso $1(w \neq 0, r \cdot s>0)$

1. $f_{w, r, s}\left(0 \times\left[0, \frac{1}{r}\right]\right)=w_{i}\left(\right.$ com $\left.<w_{i}>=w\right)$ um homeomorfismo local tal que ao "caminharmos" de $(0,0)$ para $\left(0, \frac{1}{r}\right)$ percorremos $w_{i}$ no sentido positivo, se $r>0$, ou negativo se $r<0$.

2. Para $\frac{1}{r}<p \leq 1, f_{w, r, s}(0, p)=f_{w, r, s}\left(0, p^{\prime}\right)$ com $0 \leq p^{\prime}<\frac{1}{r}$ e $p=p^{\prime}+\frac{k}{r}$ com $k \in \mathbb{N}$.

3. Para $0 \leq q \leq \frac{1}{s}, f_{w, r, s}(q, 0)=f_{w, r, s}\left(0, \frac{s q}{r}\right)$.

4. Para $\frac{1}{s}<q \leq 1, f_{w, r, s}(q, 0)=f_{w, r, s}\left(q^{\prime}, 0\right)$ com $0 \leq q^{\prime}<\frac{1}{s}$ e $q=q^{\prime}+\frac{k}{s}$ com $k \in \mathbb{N}$.

5. Para $x \neq 0 \neq y \operatorname{com}\left(y+\frac{s}{r} x\right) \leq 1, f_{w, r, s}(x, y)=f_{w, r, s}\left(0, y+\frac{s}{r} x\right)$.

6. Para $x \neq 0 \neq y \operatorname{com}\left(y+\frac{s}{r} x\right)>1, f_{w, r, s}(x, y)=f_{w, r, s}\left((y-1) \frac{r}{s}+x, 1\right)$.

Caso $2(w \neq 0, r \cdot s<0)$

1. $f_{w, r, s}\left(0 \times\left[0, \frac{1}{r}\right]\right)=w_{i}\left(\mathrm{com}<w_{i}>=w\right)$ um homeomorfismo local tal que ao "caminharmos" de $(0,0)$ para $\left(0, \frac{1}{r}\right)$ percorremos $w_{i}$ no sentido positivo, se $r>0$, ou negativo se $r<0$.

2. Para $\frac{1}{r}<p \leq 1, f_{w, r, s}(0, p)=f_{w, r, s}\left(0, p^{\prime}\right) \operatorname{com} 0 \leq p^{\prime}<\frac{1}{r}$ e $p=p^{\prime}+\frac{k}{r}$ $\operatorname{com} k \in \mathbb{N}$.

3. Para $\left(1-\frac{1}{s}\right) \leq q \leq 1, f_{w, r, s}(q, 0)=f_{w, r, s}\left(0, \frac{s}{r}(1-q)\right)$.

4. Para $0 \leq q<1, f_{w, r, s}(q, 0)=f_{w, r, s}\left(q^{\prime}, 0\right) \operatorname{com}\left(1-\frac{1}{s}\right)<q^{\prime} \leq 1 e$ $q^{\prime}=q-\frac{k}{s} \operatorname{com} k \in \mathbb{N}$.

5. Para $x \neq 0 \neq y \operatorname{com}\left((1-x) \frac{s}{r}+y\right) \leq 1, f_{w, r, s}(x, y)=f_{w, r, s}\left(1,(1-x) \frac{s}{r}+y\right)$.

6. Para $x \neq 0 \neq y \operatorname{com}\left((1-x) \frac{s}{r}+y\right)>1, f_{w, r, s}(x, y)=f_{w, r, s}\left((1-y) \frac{r}{s}+x, 1\right)$.

Caso $3(w \neq 0, s=0)$

1. $f_{w, r, s}\left(0 \times\left[0, \frac{1}{r}\right]\right)=w_{i}\left(\right.$ com $\left.\left\langle w_{i}\right\rangle=w\right)$ um homeomorfismo local tal que ao "caminharmos" de $(0,0)$ para $\left(0, \frac{1}{r}\right)$ percorremos $w_{i}$ no sentido positivo, se $r>0$, ou negativo se $r<0$. 
2. Para $\frac{1}{r}<p \leq 1, f_{w, r, s}(0, p)=f_{w, r, s}\left(0, p^{\prime}\right) \operatorname{com} 0 \leq p^{\prime}<\frac{1}{r}$ e $p=p^{\prime}+\frac{k}{r}$ com $k \in \mathbb{N}$.

3. Para $x \neq 0, f_{w, r, s}(x, y)=f_{w, r, s}(0, y)$.

Caso $4(w \neq 0, r=0)$

1. $f_{w, r, s}\left(\left[0, \frac{1}{s}\right] \times 0\right)=w_{i}\left(\right.$ com $\left.\left\langle w_{i}\right\rangle=w\right)$ um homeomorfismo local tal que ao "caminharmos" de $(0,0)$ para $\left(0, \frac{1}{s}\right)$ percorremos $w_{i}$ no sentido positivo, se $s>0$, ou negativo se $s<0$.

2. Para $\frac{1}{s}<p \leq 1, f_{w, r, s}(p, 0)=f_{w, r, s}\left(p^{\prime}, 0\right)$ com $0 \leq p^{\prime}<\frac{1}{s}$ e $p=p^{\prime}+\frac{k}{s}$ $\operatorname{com} k \in \mathbb{N}$.

3. Para $y \neq 0, f_{w, r, s}(x, y)=f_{w, r, s}(x, 0)$.

Caso $5(w=0$ ou $r=s=0)$

$f_{w, r, s}$ constante no ponto base de $S_{g}$

Observe que $<a_{1}>e<a_{2}>$ são os geradores de $\pi_{1}\left(S_{g}\right)$ e temos que:

1. $\left(f_{w, r, s}\right)_{\#}\left(<a_{1}>\right)=<w>^{r}$;

2. $\left(f_{w, r, s}\right)_{\#}\left(<a_{2}>\right)=<w>^{s}$.

Intuitivamente $f_{w, r, s}$ é definida como um homeomorfismo local sobre uma curva da classe $w$ em um pedaço do bordo do quadrado e depois estendida sobre $a_{1} \mathrm{e}$ $a_{2}$ por $r$ e $s$ "repetições" desse homeomorfismo, no interior do quadrado $f_{w, r, s}$ é constante nas retas com coeficiente angular $\frac{-s}{r}$ ( $\operatorname{casos} 1$ e 3 ) e $\frac{s}{r}$ (caso 2).

Lema 3.7. Dada $f: S_{1} \mapsto S_{g}$ com $g \geq 2$, existem $w \in \pi_{1}\left(S_{g}\right)$ e $r, s \in \mathbb{R}$ tais que $f$ é homotópica a $f_{w, r, s}$.

Demonstração: Observamos que $f_{\#}\left(\pi_{1}\left(S_{1}\right)\right)=<f_{\#}\left(a_{1}\right), f_{\#}\left(a_{2}\right)>\subset \pi_{1}\left(S_{g}\right)$ assim, pelo Freiheitssatz [MKS, Teorema 4.10, página 252], $f_{\#}\left(\pi_{1}\left(S_{1}\right)\right)$ é livre com um único gerador, portanto $f_{\#}\left(\pi_{1}\left(S_{1}\right)\right) \approx \mathbb{Z}$. Desse modo temos que existem $w \in \pi_{1}\left(S_{g}\right)$ e $r, s \in \mathbb{R}$ tais que $f_{\#}\left(<a_{1}>\right)=<w>^{r}$ e $f_{\#}\left(<a_{2}>\right)=<w>^{s}$.

Como a classe de homotopia preservando ponto base de $f$ é unicamente determinada ${ }^{1}$ por $f_{\#}$ temos que $f$ é homotópica a $f_{w, r, s}$.

Teorema 3.8. Se $g \geq 2, B(g, 1,0,0)=1$.

\footnotetext{
${ }^{1} S_{1}$ é um espaço $K[\pi, 1]$.
} 


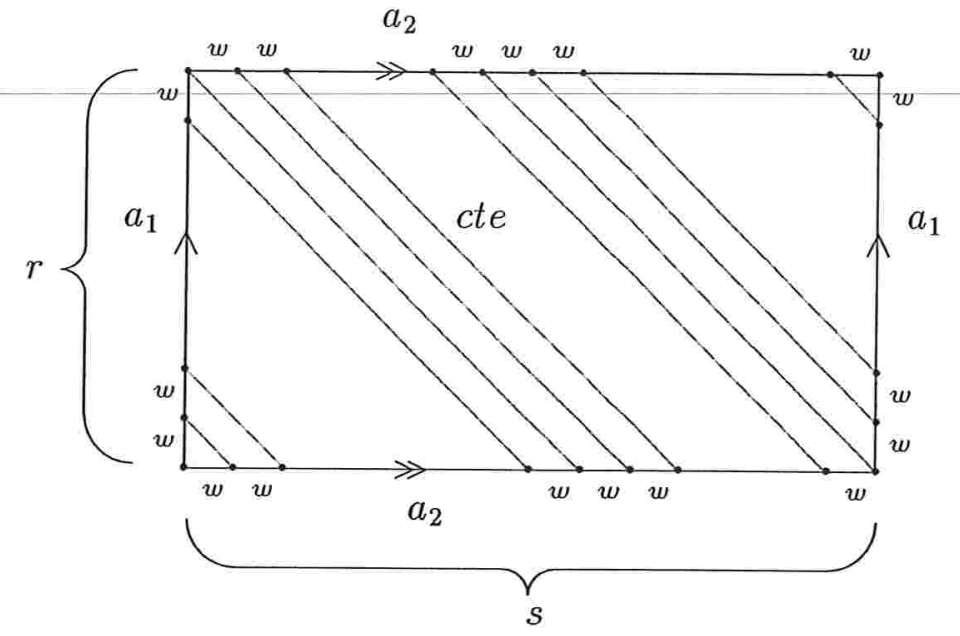

Figura 3.1: Forma padrão da $f$; caso $1(\operatorname{com} s>r)$

Demonstração: Sejam $f_{1}, f_{2}: S_{1} \mapsto S_{g}$, pelo Lema 3.7 podemos supor $f_{1}=$ $f_{w, r, s}$ e $f_{2}=f_{\alpha, p, q}$, podemos ainda supor que a imagem de $f_{1}$ e de $f_{2}$ estão contidas em duas curvas geodésicas $w_{0}$ e $\alpha_{0}$ de $S_{g}$ (ver [Stillwell]). Temos então que:

Caso $1\left(w^{k} \neq \alpha^{k^{\prime}}, \forall k, k^{\prime} \in \mathbb{Z} \operatorname{com} r q \neq s p\right)$ ou $(r=p=0)$ ou $(q=s=0)$.

Como $w \neq \alpha$ e a imagem de $f_{1}$ e de $f_{2}$ estão contidas em duas curvas geodésicas de $S_{g}$ pelo Lema 3.5 temos que $w_{0} \cap \alpha_{0}$ é finito, assim $w_{0} \cap \alpha_{0}=$ $\left\{y_{1}, y_{2}, \cdots, y_{n}\right\}$ logo

$$
\operatorname{Coin}\left(f_{1}, f_{2}\right)=\bigcup_{i=1}^{n}\left(f_{1}^{-1}\left(y_{i}\right) \cap f_{2}^{-1}\left(y_{i}\right)\right)
$$

como $f_{1}^{-1}\left(y_{i}\right)$ e $f_{2}^{-1}\left(y_{i}\right)$ são a união de um número finito de segmentos de reta com coeficientes angulares distintos (quando identificamos $S_{1}$ com o quadrado) temos que $\operatorname{Coin}\left(f_{1}, f_{2}\right)$ é finito.

Agora se $\gamma: I \mapsto S_{1}$ é uma curva tal que $\gamma(0)=c_{1}$ e $\gamma(1)=c_{2}$ com $c_{1}, c_{2} \in \operatorname{Coin}\left(f_{1}, f_{2}\right)$ temos que se $\widetilde{\gamma}: I \mapsto \mathbb{R}^{2}$ é um levantamento de $\gamma$ com $\widetilde{\gamma}(0)=\widetilde{c_{1}}$ e $\widetilde{\gamma}(1)=\widetilde{c_{2}}$ então $\widetilde{\gamma}$ é homotópica (relativamente aos seus extremos) a uma curva $\bar{\gamma}: I \mapsto \mathbb{R}^{2}$ que é um homeomorfismo cuja imagem é um segmento de reta entre $\widetilde{c_{1}}$ e $\widetilde{c_{2}}$.

Assim temos que $\gamma$ é homotópica (relativamente aos seus extremos) a $p \circ \bar{\gamma}$ : $I \mapsto S_{1}$ e $f_{i} \circ p \circ \bar{\gamma}$ é constante ou um homeomorfismo local para $i=1,2$.

Considerando $y_{i_{1}}=f_{1}\left(c_{1}\right)=f_{2}\left(c_{1}\right)$ como o ponto base de $S_{g}$ temos que $f_{1}$ e $f_{2}$ admitem levantamentos $\widetilde{f_{1}}, \widetilde{f}_{2}: \mathbb{R}^{2} \mapsto \mathbb{D}^{2}$ com $\widetilde{f_{1}}\left(\widetilde{c_{1}}\right)=\widetilde{f_{2}}\left(\widetilde{c_{1}}\right)=\widetilde{y_{i_{1}}}$ onde 
$p\left(\widetilde{y_{i_{1}}}\right)=y_{i_{1}}$ e com $\tilde{f}_{i} \circ \bar{\gamma}, i=1,2$, constante ou um homeomorfismo local com imagem em um segmento de reta (hiperbólico) $\widetilde{w_{0}}$ ou $\widetilde{\alpha_{0}}$.

Como $\widetilde{w_{0}} \cap \widetilde{\alpha_{0}}=\widetilde{c_{1}}$ temos que $\tilde{f}_{1}\left(\widetilde{c_{2}}\right) \neq \widetilde{f_{2}}\left(\widetilde{c_{2}}\right)$ o que mostra que $c_{1}$ e $c_{2}$ não estão na mesma classe de coincidência do par $\left(f_{1}, f_{2}\right)$.

Observamos, agora, que se $c \in \operatorname{Coin}\left(f_{1}, f_{2}\right)$ podemos definir ${ }^{2}$ uma vizinhança euclidiana $U$ de $c$ onde $f_{1}$ e $f_{2}$ se comportam como projeções $p(x, y)=( \pm x, 0)$ e $p(x, y)=(0, \pm y)$, em qualquer dessas situações $\left.\left(f_{1}-f_{2}\right)\right|_{U}$ é um homeomorfismo, portanto $\mid$ ind $(c) \mid=1$ o que encerra o caso 1 .

Caso $2\left(w^{k} \neq \alpha^{k^{\prime}}, \forall k, k^{\prime} \in \mathbb{Z} \operatorname{com} r q=s p\right)$

Como no caso anterior temos que $w_{0} \cap \alpha_{0}=\left\{y_{1}, y_{2}, \cdots, y_{n}\right\}$ e

$$
\operatorname{Coin}\left(f_{1}, f_{2}\right)=\bigcup_{i=1}^{n}\left(f_{1}^{-1}\left(y_{i}\right) \cap f_{2}^{-1}\left(y_{i}\right)\right)
$$

mas nesse caso $f_{1}^{-1}\left(y_{i}\right)=f_{2}^{-1}\left(y_{i}\right), \quad i=1,2, \cdots, n$.

Com uma pequena translação de $f_{2}$ no sentido de $a$ temos $\operatorname{Coin}\left(f_{1}, f_{2}\right)=\emptyset$. Isso mostra que se $C$ é uma classe de coincidência do par $\left(f_{1}, f_{2}\right)$ temos que $\operatorname{ind}(C)=0$.

Caso $3\left(\exists k, k^{\prime} \in \mathbb{Z}\right.$ com $\left.w^{k}=\alpha^{k^{\prime}}\right)$

Temos então que existe $\beta \in \pi_{1}\left(S_{g}\right)$ tal que:

$$
\begin{array}{ll}
f_{1}\left(a_{1}\right)=\beta^{r^{\prime}} & f_{1}\left(a_{2}\right)=\beta^{s^{\prime}} \\
f_{2}\left(a_{1}\right)=\beta^{p^{\prime}} & f_{2}\left(a_{2}\right)=\beta^{q^{\prime}}
\end{array}
$$

Podemos novamente supor que $f_{1}=f_{\beta, r^{\prime}, s^{\prime}}$ e $f_{2}=f_{\beta, p^{\prime}, q^{\prime}}$ ambas com imagem sobre um laço $\beta_{0} \in S_{g}\left(<\beta_{0}>=\beta\right)$.

Se $r^{\prime} q^{\prime}=s^{\prime} p^{\prime}$ podemos tomar $\beta_{1}$ uma pequena perturbação de $\beta$ de modo que $\beta \cap \beta_{1}$ seja finito, nesse caso temos $f_{2}$ é homotópica a uma $f_{2}^{\prime}: S_{1} \mapsto S_{g}$ também da forma $f_{\beta, p^{\prime}, q^{\prime}}$ mas com imagem em $\beta_{1}$. O mesmo argumento usado no caso 2 mostra que é possível tomar $f_{2}^{\prime}$ tal que $C \operatorname{cin}\left(f_{1}, f_{2}^{\prime}\right)=\emptyset$ e portanto $\operatorname{ind}(C)=0$ par toda classe de coincidência $C$ do par $\left(f_{1}, f_{2}\right)$.

Se $r^{\prime} q^{\prime} \neq s^{\prime} p^{\prime}$ tomamos $\widetilde{\beta_{0}} \in \mathbb{D}^{2}$ um levantamento de $\beta_{0}$ com $\widetilde{\beta_{0}}$ contido em uma reta (hiperbólica) $\lambda$, tomando-se o ponto base de $S_{g}, y_{0}$ de modo que $\widetilde{y_{0}}$ seja um dos extremos de $\widetilde{\beta_{0}}$ podemos identificar (ver [Stillwell, página 158]) $\beta \in \pi_{1}\left(S_{g}\right)$ com uma translação de $\mathbb{D}^{2}, \lambda^{*}$ com eixo em $\lambda$.

\footnotetext{
${ }^{2}$ Note que $c$ é um ponto onde duas pré-imagens de um mesmo ponto, se encontram transversalmente.
} 
Dado $\epsilon>0$ tomamos $W=\left\{\widetilde{y} \in \mathbb{D}^{2} \mid \operatorname{dist}(\widetilde{y}, \lambda)=\epsilon\right\}^{3}$ e escolhendo $\widetilde{y_{1}} \in W$ tal que $\operatorname{dist}\left(\widetilde{y_{0}}, \widetilde{y_{1}}\right)=\epsilon$ podemos definir $\lambda_{1}$ como sendo a componente conexa de $W$ que contem $\widetilde{y_{1}}$.

Por [Stillwell, página 158] temos que $\lambda_{1}$ é invariante por $\lambda^{*}$, tomamos então:

$$
\widetilde{\beta_{1}}=\left\{\widetilde{y} \in \lambda_{1} \mid \operatorname{dist}\left(\widetilde{y}, \widetilde{\beta_{0}}\right)=\epsilon\right\} \text {. }
$$

Observamos que $\widetilde{y_{1}}$ e $\lambda^{*}\left(\widetilde{y_{1}}\right)$ são os extremos de $\widetilde{\beta_{1}}$ (ver figura 3.2 ), além disso, $q\left(\widetilde{y_{1}}\right)=q\left(\lambda^{*}\left(\widetilde{\lambda_{1}}\right)\right)$, portanto $q\left(\widetilde{\beta_{1}}\right)$ é um laço em $S_{g}$ e tomando-se $\epsilon$ suficientemente pequeno podemos supor que $\left\langle q\left(\widetilde{\beta_{1}}\right)\right\rangle=\beta$, ou seja $\beta_{0}$ e $q\left(\widetilde{\beta_{1}}\right)$ são curvas livremente homotópicas.

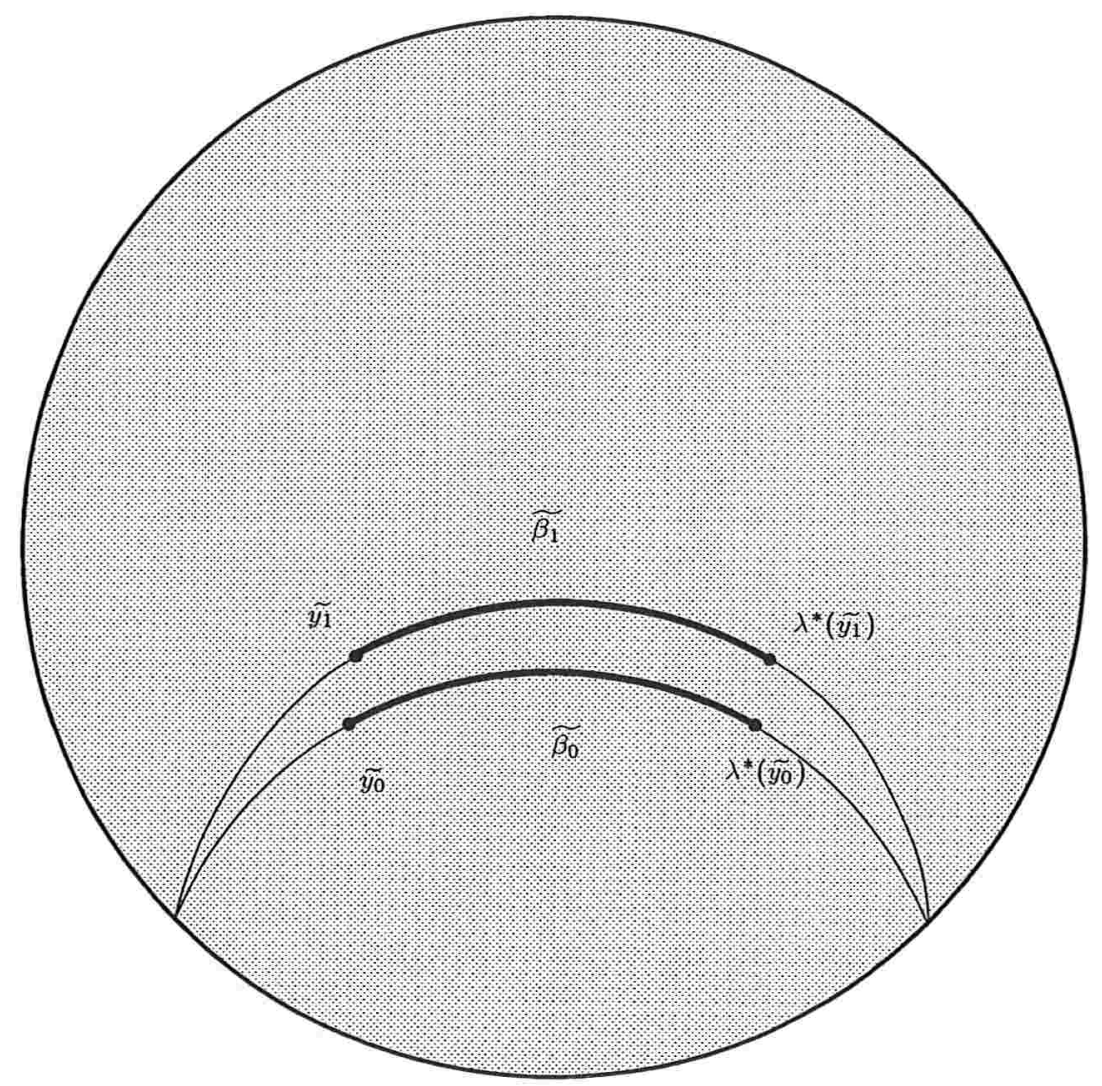

Figura 3.2: Levantamento $\widetilde{\beta_{0}}$ de $\beta_{0}$

Agora se $x_{0} \in \operatorname{Coin}\left(f_{1}, f_{2}\right)$ tomamos $f_{1}\left(x_{0}\right)=y_{0}$ como ponto base de $S_{g}$, temos que $f_{1}\left(S_{1}\right) \subset \beta_{0}, f_{2}\left(S_{1}\right) \subset \beta_{0}$ e o par $f_{1}, f_{2}$ admite levantamentos $\widetilde{f}_{i}: S_{1} \mapsto \mathbb{D}^{2}$, tais que $\widetilde{f}_{i}\left(S_{1}\right) \subset \lambda, i=1,2$.

\footnotetext{
${ }^{3} \mathrm{~A}$ distância usada é a hiperbólica
} 
Como $\lambda_{1}$ é invariante por $\lambda^{*}$ podemos criar uma homotopia $H(x, t): S_{1} \times I \mapsto$ $\mathbb{D}^{2}$ tal que $H(x, 0)=\widetilde{f}_{2}, H(x, 1)$ tem sua imagem em $\lambda_{1}, H\left(x_{0}, I\right)$ percorre o segmento de reta $\kappa$ que une $\widetilde{y_{0}}$ até $\widetilde{y_{1}}$ e $H\left(\lambda^{*}\left(x_{0}\right), I\right)$ percorre $\lambda^{*}(\kappa)$, como $\beta_{0}$ e $q\left(\widetilde{\beta_{1}}\right)$ são curvas livremente homotópicas temos que $q(H(x, t))$ é uma homotopia em $S_{g}$ entre $f_{2}$ e $f_{2}^{\prime}=H(x, 1)$, observamos agora que ao final da homotopia as imagens dos levantamentos de $f_{1}$ e $f_{2}^{\prime}$ são disjuntas, assim a classe de coincidência $C$ que contém $x_{0}$ é não essencial, portanto ind $(C)=0$. 


\section{O caso não orientável}

Nesse capítulo apresentamos alguns resultados sobre a limitação de índices de classes de coincidência quando pelo menos uma das superfícies é não orientável. As definições de grau de aplicação e de índice de coincidências podem ser encontradas no capítulo 2 (Preliminares), usaremos a notação e as definições ali apresentadas. Em todos os casos estudados $f_{2}$ será fiel à orientação o que nos permite definir $L\left(f_{1}, f_{2}\right)$ e $\operatorname{ind}\left(f_{1}, f_{2}, C\right)$.

\subsection{Aplicações de $K_{h} \mapsto K_{g}$}

Definimos de maneira análoga ao caso orientável:

$$
B_{K}\left(g, h, d_{1}, d_{2}\right)=\sup \left\{\left|\operatorname{ind}\left(f_{1}, f_{2}, C\right)\right||| \operatorname{deg}\left(f_{1}\right)\left|=d_{1},\right| \operatorname{deg}\left(f_{2}\right) \mid=d_{2}\right\},
$$

onde o supremo é tomado para todas as classes de coincidência $C$ de todos os pares de aplicações ${ }^{1}\left(f_{1}, f_{2}\right): K_{h} \mapsto K_{g}$ com tais graus e $f_{2}$ fiel à orientação.

\subsubsection{A garrafa de Klein}

Fixaremos: $\pi_{1}(K)=<\alpha, \beta \mid \alpha \cdot \beta \cdot \alpha \cdot \beta^{-1}>, p: S_{1} \mapsto K$ a aplicação de recobrimento do toro na garrafa de Klein com $\pi_{1}\left(S_{1}\right)=\left\langle a, b \mid a \cdot b \cdot a^{-1} \cdot b\right\rangle$, assim $p(a)=\alpha$ e $p(b)=\beta^{2}$.

Teorema 4.1. $-1 \leq B_{K}\left(0,0, d_{1}, d_{2}\right) \leq 1, \forall \quad d_{1}, d_{2} \geq 0$.

Demonstração: Tomemos $f_{1}, f_{2}: K \mapsto K \operatorname{com} f_{2}$ fiel à orientação assim: $\frac{f_{2 \#}(\alpha)=\alpha^{r} \text { e } f_{2 \#}(\beta)=\alpha^{s} \beta^{2 q+1} \text {. }}{{ }^{1} \text { Para a defin }}$

\footnotetext{
${ }^{1}$ Para a definição de $K_{h}$ ver a seção 2.5 na página 40 .
} 
Caso $1 f_{1}$ não é fiel à orientação.

Temos que $f_{1 \#}(\alpha)=1, f_{1}(\beta)=\alpha^{t} \beta^{2 p}$ e $f_{1}, f_{2}$ se levantam para aplicações $\widetilde{f}_{1}, \widetilde{f}_{2}: S_{1} \mapsto S_{1}$ tais que: $\widetilde{f}_{1 \#}(a)=1, \widetilde{f}_{1 \#}(b)=a^{2 t} b^{2 p}, \widetilde{f}_{2 \#}(a)=a^{r}, \widetilde{f}_{2 \#}(b)=$ $b^{2 q+1}$.

Note que a identidade da garrafa de Klein se levanta para a identidade ou para uma aplicação $\theta: S_{1} \mapsto S_{1} \operatorname{com} \theta(a)=a^{-1}$ e $\theta(b)=b$ (para detalhes ver [Gonçalves \& Kiihl, Proposição 10.7, página 86]).

Seja $x_{0} \in \operatorname{Coin}\left(f_{1}, f_{2}\right)$ e $\widetilde{x_{0}}, \widetilde{x_{0}}{ }^{\prime} \in S_{1} \operatorname{com} p\left(\widetilde{x_{0}}\right)=p\left(\widetilde{x_{0}}{ }^{\prime}\right)=x_{0}$, se $\widetilde{x_{0}} \in$ $\operatorname{Coin}\left(\widetilde{f}_{1}, \widetilde{f}_{2}\right)$ e $\gamma$ é uma curva em $S_{1}$ com início em $\widetilde{x_{0}}$ e término em $\widetilde{x_{0}}{ }^{\prime}$ temos que $p(\gamma) \in \frac{\pi_{1}(K)}{p_{\#}\left(\pi_{1}\left(S_{1}\right)\right)}, \operatorname{assim} \widetilde{x_{0}} \in \operatorname{Coin}\left(\widetilde{f}_{1}, \widetilde{f}_{2}\right)$ se e somente se $\widetilde{f}_{1}(\gamma) \circ \widetilde{f}_{2}(\gamma)^{-1} \in$ $\pi_{1}\left(S_{1}\right)$ ou seja $f_{1 \#}(p(\gamma)) \circ f_{2 \#}(p(\gamma))^{-1} \in p_{\#}\left(\pi_{1}\left(S_{1}\right)\right)$.

Mas como $\widetilde{x_{0}}$ e $\widetilde{x_{0}^{\prime}}$ estão na mesma fibra de $p, p(\gamma)=\alpha^{k} \beta^{2 l+1}$ e temos que

$$
\begin{aligned}
f_{1 \#}(p(\gamma)) \circ f_{2 \#}(p(\gamma))^{-1} & =\alpha^{t(2 l+1)} \beta^{(2 l+1)(2 p)} \beta^{-(2 l+1)(2 q+1)} \alpha^{-k r-s} \\
& =\alpha^{k^{\prime}} \beta^{2 q^{\prime}+1} \notin p_{\#}\left(\pi_{1}\left(S_{1}\right)\right) .
\end{aligned}
$$

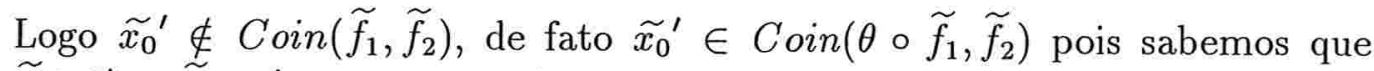
$\widetilde{f}_{1}\left(\widetilde{x_{0}}\right)$ e $\widetilde{f_{2}}\left(\widetilde{x_{0}}{ }^{\prime}\right)$ estão em $p^{-1}\left(f_{1}\left(x_{0}\right)\right)$ que só tem dois elementos. Assim as classes de coincidência de $f_{1}, f_{2}$ estão em correspondência biunívoca com as classes de coincidência de $\widetilde{f}_{1}, \widetilde{f}_{2}$ através da $p$.

Como $p$ é fiel à orientação se $C$ é uma classes de coincidência de $f_{1}, f_{2}$, $\left|\operatorname{ind}\left(f_{1}, f_{2}, C\right)\right|=\left|\operatorname{ind}\left(\widetilde{f}_{2}, \widetilde{f}_{1}, p^{-1}(C)\right)\right| \operatorname{mas}-1 \leq\left|\operatorname{ind}\left(\widetilde{f}_{1}, \widetilde{f}_{2}, p^{-1}(C)\right)\right| \leq 1$ [Brooks, página 125] e portanto $-1 \leq\left|\operatorname{ind}\left(f_{1}, f_{2}, C\right)\right| \leq 1$.

Caso $2 f_{1}$ também é fiel à orientação.

Nesse caso temos que $f_{1 \#}(\alpha)=\alpha^{m}, f_{1}(\beta)=\alpha^{t} \beta^{2 p+1}$ e $f_{1}, f_{2}$ se levantam para aplicações $\widetilde{f}_{1}, \widetilde{f}_{2}: S_{1} \mapsto S_{1}$ tais que: $\widetilde{f}_{1 \#}(a)=a^{m}, \widetilde{f}_{1 \#}(b)=b^{2 p+1}$, $\widetilde{f}_{2 \#}(a)=a^{r}, \widetilde{f}_{2 \#}(b)=b^{2 q+1}$.

Fazendo o mesmo raciocínio do caso anterior obtemos que se $\widetilde{x_{0}} \in \operatorname{Coin}\left(\widetilde{f}_{1}, \widetilde{f}_{2}\right)$ então $\widetilde{x_{0}}{ }^{\prime} \in \operatorname{Coin}\left(\widetilde{f_{1}}, \widetilde{f_{2}}\right)$ também, se $\widetilde{x_{0}}$ e $\widetilde{x_{0}}{ }^{\prime}$ não estiverem na mesma classe de coincidência de $\widetilde{f}_{1}, \widetilde{f}_{2}$ então teremos que se $C$ é a classe que contem $x_{0}$ então $\left|\operatorname{ind}\left(f_{1}, f_{2}, C\right)\right|=\left|\operatorname{ind}\left(\tilde{f}_{1}, \widetilde{f}_{2}, \tilde{C}\right)\right|$ onde $\tilde{C}$ é a classe de coincidência de $\widetilde{f_{1}}, \widetilde{f_{2}}$ que contem $\widetilde{x_{0}}$.

Obsevamos que se $\widetilde{x_{0}}$ e $\widetilde{x_{0}}{ }^{\prime}$ estão na mesma classe de coincidência ${ }^{2}$ todos os pares $\tilde{x_{1}}, \tilde{x_{2}}$ de pontos tais que $p\left(\tilde{x_{1}}\right)=p\left(\tilde{x_{2}}\right) \in \operatorname{Coin}\left(f_{1}, f_{2}\right)$ estão, os dois, na mesma classe de $\widetilde{f}_{1}, \widetilde{f}_{2}$ ou de $\theta \circ \widetilde{f}_{1}, \widetilde{f}_{2}$.

\footnotetext{
${ }^{2}$ Então existe uma curva $\gamma$ em $S_{1}$ com início em $\widetilde{x_{0}}$ e término em $\widetilde{x_{0}}{ }^{\prime}$ tal que $\widetilde{f_{1}}(\gamma) \circ \widetilde{f}_{2}(\gamma)^{-1}$ é homotopicamente trivial, mas nesse caso $f_{2 \#}(p(\gamma)) \circ f_{1 \#}(p(\gamma))^{-1}$ também será homotopicamente
} 
Como $p$ é um homeomorfismo local fiel à orientação se $\tilde{C}$ é a classe de coincidência do par $\widetilde{f}_{1}, \widetilde{f}_{2}$ (ou de $\left.\theta \circ \widetilde{f_{1}}, \widetilde{f}_{2}\right)$ que contem $\widetilde{x_{0}},\left|i n d\left(\widetilde{f}_{1}, \widetilde{f}_{2}, \tilde{C}\right)\right|=$ $2\left|\operatorname{ind}\left(f_{1}, f_{2}, C\right)\right|\left(\right.$ ou $\left.\left|\operatorname{ind}\left(\theta \circ \widetilde{f}_{1}, \widetilde{f}_{2}, \tilde{C}\right)\right|=2\left|\operatorname{ind}\left(f_{1}, f_{2}, C\right)\right|\right)$.

Como $\left|\operatorname{ind}\left(f_{1}, f_{2}, C\right)\right|$ é inteiro [Gonçalves \& Jezierski, Remark 2, página 22] $\mathrm{e}-1 \leq\left|\operatorname{ind}\left(\widetilde{f}_{1}, \widetilde{f}_{2}, \tilde{C}\right)\right|,\left|\operatorname{ind}\left(\theta \circ \widetilde{f}_{1}, \widetilde{f}_{2}, \tilde{C}\right)\right| \leq 1$ temos que $\operatorname{ind}\left(f_{2}, f_{1}, C\right)=0$.

Definição 4.2. ${ }^{3}$ Dados $w \in \pi_{1}\left(K_{g}\right), s \in \mathbb{Z}$ e tomando-se a garrafa de Klein como o quadrado $[0,1] \times[0,1]$ com os lados identificados, ${ }^{\alpha{ }^{\beta}{ }^{\beta}{ }^{\alpha}}{ }^{\alpha}$, definimos $f_{w, s}: K \mapsto K_{g}$ como:

1. $f_{w, s}\left(\left[0, \frac{1}{s}\right] \times 0\right)=\bar{w}($ com $<\bar{w}>=w)$ um homeomorfismo local tal que ao "caminharmos" de $(0,0)$ para $\left(\frac{1}{s}, 0\right)$ percorremos $\bar{w}$ no sentido positivo, se $s>0$, ou negativo se $s<0$.

2. Para $\frac{1}{s}<q \leq 1, f_{w, s}(q, 0)=f_{w, s}\left(q^{\prime}, 0\right)$ com $0 \leq q^{\prime}<\frac{1}{s}$ e $q=q^{\prime}+\frac{k}{s}$ com $k \in \mathbb{N}$.

3. Para $y>0, f_{w, s}(x, y)=f_{w, s}(x, 0)$.

Observe que a pré-imagem de um ponto qualquer de $K_{g}$ por uma aplicação na forma $f_{w, s}$ é a união de u número finito de segmentos de retas verticais ou é vazia.

Teorema 4.3. ${ }^{4} B_{K}(g, 0,0,0)=0$ para $g \geq 1$.

Demonstração: Seja $f: K \mapsto K_{g}$ uma aplicação, Sabemos que $\pi_{1}\left(K_{g}\right)$ é um grupo que pode ser descrito por $2 g+2$ geradores e uma única relação:

$$
\pi_{1}\left(K_{g}\right)=<a_{1}, b_{1}, a_{2}, b_{2}, \ldots, a_{g}, b_{g}, \alpha, \beta \mid \prod_{i=1}^{g}\left[a_{i}, b_{i}\right] \cdot \alpha \cdot \beta \cdot \alpha \cdot \beta^{-1}>
$$

Observamos que $f_{\#}\left(\pi_{1}\left(K_{g}\right)\right)$ é gerado por dois elementos $w^{\prime}=f_{\#}(\alpha)$ e $w^{\prime \prime}=f_{\#}(\beta)$ que satisfazem a relação:

$$
w^{\prime} \cdot w^{\prime \prime} \cdot w^{\prime} \cdot w^{\prime \prime-1}=1
$$

trivial, como $p(\gamma)=\alpha^{k} \beta^{2 l+1}$ temos que:

$$
\begin{aligned}
f_{1 \#}(p(\gamma)) \circ f_{2 \#}(p(\gamma))^{-1} & =\alpha^{k m+t} \beta^{(2 l+1)(2 p+1)} \beta^{-(2 l+1)(2 p+1)} \alpha^{-k r} \\
& =\alpha^{k(m-r)+t-s} \beta^{(2 l+1)(2 p-2 q)} .
\end{aligned}
$$

De onde concluímos que $p=q$, nesse caso teremos $L\left(\tilde{f}_{1}, \tilde{f}_{2}\right)=0$ o que nos garante que todas as classes de coincidência do par $\tilde{f}_{1}, \widetilde{f}_{2}$ têm índice zero.

${ }^{3}$ Compare com a Definição 3.6

${ }^{4}$ Compare com o Teorema 3.8 
Usando Freiheitssatz [MKS, Teorema 4.10, página 252] temos que $f_{\#}\left(\pi_{1}(K)\right)$ é livre com um único gerador, assim existe $w \in \pi_{1}\left(K_{g}\right)$ tal que $w^{\prime}=w^{r}$ e $w^{\prime \prime}=w^{s}$ $\operatorname{com} r, s \in \mathbb{Z}$ e temos, pela relação acima:

$$
w^{r} \cdot w^{s} \cdot w^{r} \cdot w^{-s}=1
$$

assim $r=0$ e $f_{\#}(\alpha)=1$ o elemento trivial, dessa forma, como $K$ é um espaço de Eilenberg-MacLane do tipo $K\left[\pi_{1}\right], f$ é homotópica a uma aplicação $f_{w, s}$.

Tomando-se um par $f_{1}, f_{2}: K \mapsto K_{g}$ temos que $f_{1} \sim f_{w_{1}, s}$ e $f_{2} \sim f_{w_{2}, r}$ escolhendo $\overline{w_{1}}, \overline{w_{2}}$ curvas em $K_{g}$ tais que: $\left\langle\overline{w_{1}}\right\rangle=w_{1},\left\langle\overline{w_{2}}\right\rangle=w_{2}$ e $\overline{w_{1}} \cap \overline{w_{2}}=$ $\left\{p_{1}, p_{2}, \cdots, p_{n}\right\}$ seja finito podemos supor que

$$
f_{w_{1}, s}(K) \subset \overline{w_{1}} \text { e } f_{w_{2}, r}(K) \subset \overline{w_{2}}
$$

nessas condições:

$$
\operatorname{Coin}\left(f_{w_{1}, s}, f_{w_{2}, r}\right)=\bigcup_{i=1}^{n}\left(f_{w_{1}, s}^{-1}\left(p_{i}\right) \cap f_{w_{2}, r}^{-1}\left(p_{i}\right)\right)
$$

De fato se $\operatorname{Coin}\left(f_{w_{1}, s}, f_{w_{2}, r}\right) \neq \emptyset$ com uma translação suficientemente pequena de $f_{w_{2}, r}$ no sentido de $\beta$ teremos $C \operatorname{oin}\left(f_{w_{1}, s}, f_{w_{2}, r}\right)=\emptyset$. Isso mostra que se $C$ é uma classe de coincidência do par $\left(f_{w_{1}, s}, f_{w_{2}, r}\right)$ temos que ind $\left(f_{w_{1}, s}, f_{w_{2}, r}, C\right)=0$; como $f_{1} \sim f_{w_{1}, s}$ e $f_{2} \sim f_{w_{2}, r}$ o mesmo vale para o par $f_{1}, f_{2}$.

\subsection{2 $h \geq 1$}

Teorema 4.4. $B_{K}\left(0, h, d_{1}, d_{2}\right)=\infty$ para $h>1, \forall d_{1}, d_{2} \geq 0$.

Demonstração: Com as mesmas considerações feitas no início da demonstração do Teorema 3.3 (página 44) e lembrando que

$$
\pi_{1}\left(K_{h}\right)=<a_{1}, b_{1}, a_{2}, b_{2} \ldots, a_{h}, b_{h}, \alpha, \beta \mid \prod_{i=1}^{h}\left[a_{i}, b_{i}\right] \cdot \alpha \cdot \beta \cdot \alpha \cdot \beta^{-1}>
$$

podemos definir $\bar{f}_{i}: \pi_{1}\left(K_{h}\right) \mapsto \pi_{1}(K)$ homomorfismos ${ }^{5}$ tais que:

${ }^{5}$ Observe que $\bar{f}_{i}\left(\prod_{i=1}^{h}\left[a_{i}, b_{i}\right] \cdot \alpha \cdot \beta \cdot \alpha \cdot \beta^{-1}\right)=1$ 


$\begin{array}{llllll} & \bar{f}_{1} & & & \bar{f}_{2} \\ a_{1} & \mapsto 1 & & a_{1} & \mapsto & 1 \\ b_{1} & \mapsto \alpha_{1} & & b_{1} & \mapsto & 1 \\ a_{2} & \mapsto 1 & & a_{2} & \mapsto & \alpha^{n} \\ b_{2} & \mapsto \beta^{2} & & b_{2} & \mapsto & 1 \\ a_{3} & \mapsto 1 & & a_{3} & \mapsto & 1 \\ b_{3} & \mapsto 1 & & b_{3} & \mapsto & 1 \\ \vdots & \vdots & \vdots & \vdots & \vdots & \vdots \\ a_{h} & \mapsto 1 & & a_{h} & \mapsto & 1 \\ b_{h} & \mapsto 1 & & b_{h} & \mapsto & 1 \\ \alpha & \mapsto \alpha^{d_{1}} & & \alpha & \mapsto & \alpha^{d_{2}} \\ \beta & \mapsto \beta^{-1} . & & \beta & \mapsto & \beta\end{array}$

Como $K_{h}$ é um espaço de Eilenberg-MacLane do tipo $K\left[\pi_{1}\right]$ existem $f_{i}: K_{h} \mapsto$ $K \operatorname{com} f_{i \#}=\bar{f}_{i}$.

Observamos que $f_{1}$ e $f_{2}$ são fiéis à orientação e admitem levantamentos: $\widetilde{f}_{i}$ : $S_{2 h+1} \mapsto S_{1}$ para os recobrimentos duplos orientáveis que podem sem descritos pelos seus homomorfismos induzidos no grupo fundamental $\widetilde{f}_{i \#}: \pi_{1}\left(S_{2 h+1}\right) \mapsto \pi_{1}\left(S_{1}\right)$ como:

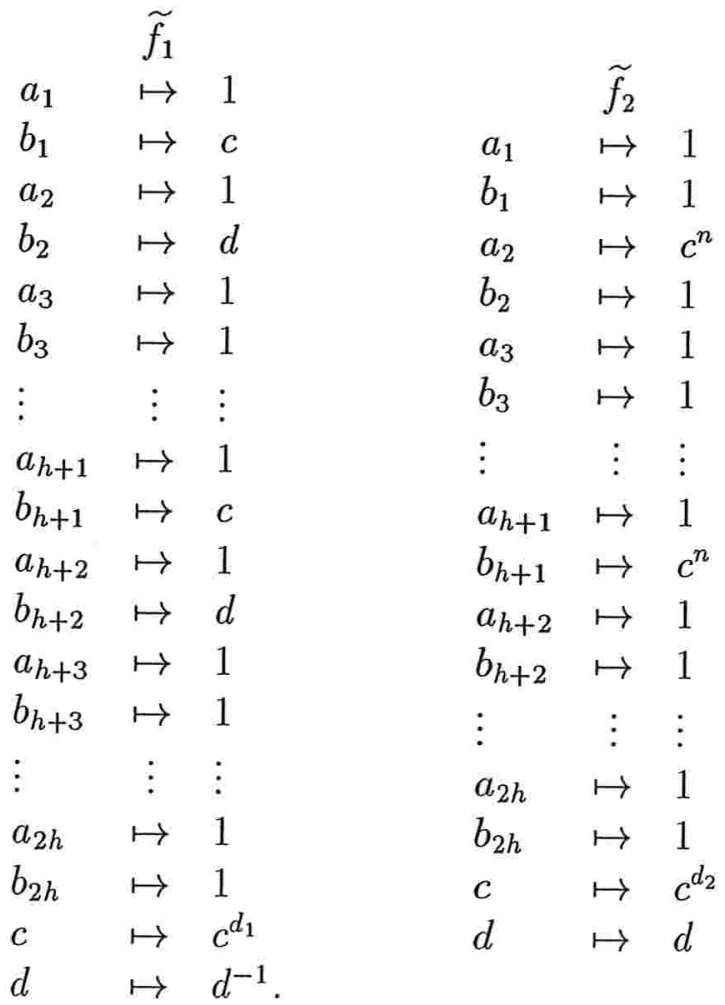


Onde $p: S_{2 h+1} \mapsto K_{h}$ é o recobrimento duplo orientável de $K_{h}, \pi_{1}\left(S_{2 h+1}\right)$ é dado por $<a_{1}, b_{1}, a_{2}, b_{2}, \ldots, a_{2 h}, b_{2 h}, c, d \mid \prod_{i=1}^{h}\left[a_{1}, b_{1}\right] \cdot[c, d]>$ e $p$ pode ser descrita
por:

- $p\left(a_{i}\right)=a_{i}, p\left(b_{i}\right)=b_{i}$ se $i \leq h$;

- $p\left(a_{i}\right)=a_{i-h}, p\left(b_{i}\right)=b_{i-h}$ se $i>h$;

- $p(c)=\alpha$;

- $p(d)=\beta^{2}$. $d_{i}$

Verificamos que $\widetilde{f}_{i}$ tem grau $d_{i}$ e assim ([GKZ, Definição 1.3, página 5]) $\operatorname{deg}\left(f_{i}\right)=$

Notemos que ${ }^{6}: f_{2}^{*}(\tilde{\alpha})=n \tilde{a_{2}}+d_{2} \tilde{\alpha}, f_{2}^{*}(\beta)=\beta$ e $f_{1}^{*}(\beta)=2 b_{2}-\beta$. Usando Teorema 2.26 , página 41 , temos que:

$$
L\left(f_{1}, f_{2}\right)=2 \cdot\left(d_{1}-n\right) .
$$

Mas sabemos que se $w \in \pi_{1}(K), w=\alpha^{p} \cdot \beta^{q}$ e temos:

$$
w=\alpha^{p} \cdot \beta^{q} \sim f_{1}\left(a_{2}^{-p}\right) \cdot \alpha^{p} \cdot \beta^{q} \cdot\left(f_{2}\left(a_{2}^{-p}\right)\right)^{-1}=\alpha^{-p} \cdot \alpha^{p} \cdot \beta^{q}=\beta^{q}
$$

Como $q=2 q^{\prime}$ ou $q=2 q^{\prime}+1 \mathrm{e}$

$$
\beta^{q} \sim f_{1}\left(\beta^{-q^{\prime}}\right) \cdot \beta^{q} \cdot\left(f_{2}\left(\beta^{-q^{\prime}}\right)\right)^{-1}=\beta^{-q^{\prime}} \cdot \beta^{q} \cdot \beta^{-q^{\prime}}
$$

temos que se $q=2 q^{\prime}$ então $\beta^{q} \sim 1$ e se $q=2 q^{\prime}+1$ temos $\beta^{q} \sim \beta$.

Temos portanto somente duas classes de Reidemeister cuja soma dos índices é $\left|2 \cdot\left(d_{1}-n\right)\right|$ [Gonçalves \& Jezierski, Teorema 4.5, página 13; Definição 5.1, página 19; Teorema 5.5, página 21] o que significa $B_{K}\left(0, h, d_{1}, d_{2}\right)=\infty$.

Teorema 4.5. $B_{K}(g, h, 0,0)=\infty$ para $h, g \geq 1$.

\section{Demonstração:}

Caso $1(g=h=1)$ Usando as mesmas notações acima, definimos $f_{1}, f_{2}: K_{1} \mapsto K_{1}$ satisfazendo ${ }^{7}$ :

\footnotetext{
${ }^{6}$ usando a notação de [Gonçalves \& Oliveira]

${ }^{7}$ Novamente observe que $f_{i \#}\left(\left[a_{1}, b_{1}\right] \cdot \alpha \cdot \beta \cdot \alpha \cdot \beta^{-1}\right)=1$
} 


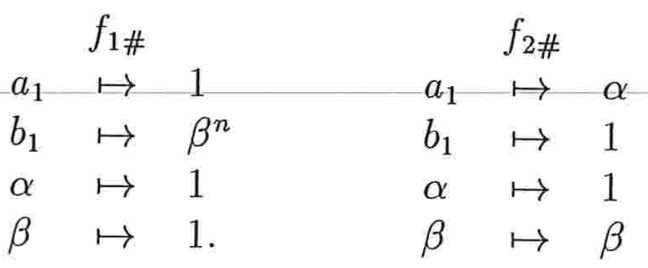

Observamos que se $w$ é uma classe de Reidemeister associada a uma classe de Nielsen $w$ é uma palavra em $\alpha$ e $\beta$ e temos:

$$
\begin{aligned}
& w=w^{\prime} \cdot \alpha^{k} \sim f_{1}\left(a_{1}^{k}\right) \cdot w^{\prime} \cdot \alpha^{k} \cdot\left(f_{2}\left(a_{1}^{k}\right)\right)^{-1} \sim w^{\prime} \cdot \alpha^{k} \cdot \alpha^{-k} \sim w^{\prime} . \\
& w=w^{\prime} \cdot \beta^{k} \sim f_{1}\left(\beta^{k}\right) \cdot w^{\prime} \cdot \beta^{k} \cdot\left(f_{2}\left(\beta^{k}\right)\right)^{-1} \sim w^{\prime} \cdot \beta^{k} \cdot \beta^{-k} \sim w^{\prime} .
\end{aligned}
$$

Assim $w \sim 1$, o que nos garante que o par $\left(f_{1}, f_{2}\right)$ tem uma única classe de Nilsen; $C$.

Na notação de [Gonçalves \& Oliveira] temos que:

$$
\begin{gathered}
f_{2}^{* 1}(, \mathbb{Q})=\left(\begin{array}{llll}
0 & 0 & 0 & 0 \\
0 & 0 & 0 & 0 \\
0 & 0 & 0 & 0 \\
0 & 0 & 0 & 1
\end{array}\right), \quad f_{2}^{* 1}(, \tilde{\mathbb{Q}})=\left(\begin{array}{llll}
0 & 0 & 0 & 1 \\
0 & 0 & 0 & 0 \\
0 & 0 & 0 & 0 \\
0 & 0 & 0 & 0
\end{array}\right) \\
\text { e } f_{1}^{* 1}(, \mathbb{Q})=\left(\begin{array}{llll}
0 & 0 & 0 & 0 \\
0 & 0 & 0 & n \\
0 & 0 & 0 & 0 \\
0 & 0 & 0 & 0
\end{array}\right)
\end{gathered}
$$

e pelo Teorema 2.27 , página 42 , temos que $L\left(f_{1}, f_{2}\right)=n$, assim $\left|\operatorname{ind}\left(f_{1}, f_{2}, C\right)\right|=$

\begin{tabular}{|c|c|c|c|c|c|}
\hline \multicolumn{3}{|c|}{$f_{1 \#}$} & \multicolumn{3}{|c|}{$f_{2 \#}$} \\
\hline$a_{1}$ & $\mapsto$ & 1 & $a_{1}$ & $\mapsto$ & $a_{1}$ \\
\hline$b_{1}$ & $\mapsto$ & $b_{1}^{-n}$ & $b_{1}$ & $\mapsto$ & 1 \\
\hline$a_{2}$ & $\mapsto$ & 1 & $a_{2}$ & $\mapsto$ & $b_{1}$ \\
\hline$b_{2}$ & $\mapsto$ & 1 & $b_{2}$ & $\mapsto$ & 1 \\
\hline$\alpha$ & $\mapsto$ & 1 & $\alpha$ & $\mapsto$ & 1 \\
\hline$\beta$ & $\mapsto$ & 1. & $\beta$ & $\mapsto$ & $\beta$ \\
\hline
\end{tabular}
$n$.

Caso $2\left(g=1\right.$ e $h=2$ ) Como no caso anterior definimos $f_{1}, f_{2}: K_{2} \mapsto K_{1}$ satisfazendo: 
Nesse caso se $w$ é uma classe de Reidemeister associada a uma classe de Nielsen então $w$ é um palavra em $a_{1}, b_{1}$ e $\beta$ e temos:

$$
\begin{gathered}
w=w^{\prime} \cdot a_{1}^{k} \sim f_{1}\left(a_{1}^{k}\right) \cdot w^{\prime} \cdot a_{1}^{k} \cdot\left(f_{2}\left(a_{1}^{k}\right)\right)^{-1} \sim w^{\prime} \cdot a_{1}^{k} \cdot a_{1}^{-k} \sim w^{\prime} . \\
w=w^{\prime} \cdot b_{1}^{k} \sim f_{1}\left(b_{2}^{k}\right) \cdot w^{\prime} \cdot a_{2}^{k} \cdot\left(f_{2}\left(b_{2}^{k}\right)\right)^{-1} \sim w^{\prime} \cdot b_{1}^{k} \cdot b_{1}^{-k} \sim w^{\prime} . \\
w=w^{\prime} \cdot \beta^{k} \sim f_{1}\left(\beta^{k}\right) \cdot w^{\prime} \cdot \beta^{k} \cdot\left(f_{2}\left(\beta^{k}\right)\right)^{-1} \sim w^{\prime} \cdot \beta^{k} \cdot \beta^{-k} \sim w^{\prime} .
\end{gathered}
$$

Novamente $w \sim 1$ e temos uma única classe de Nilsen com índice $L\left(f_{1}, f_{2}\right)$, como:

$$
\begin{aligned}
f_{2}^{* 1}(, \mathbb{Q})=\left(\begin{array}{llllll}
1 & 0 & 0 & 0 & 0 & 0 \\
0 & 0 & 0 & 0 & 0 & 0 \\
0 & 1 & 0 & 0 & 0 & 0 \\
0 & 0 & 0 & 0 & 0 & 0 \\
0 & 0 & 0 & 0 & 0 & 0 \\
0 & 0 & 0 & 0 & 0 & 1
\end{array}\right), f_{2}^{* 1}(, \tilde{\mathbb{Q}})=\left(\begin{array}{lllllll}
1 & 0 & 0 & 0 & 0 & 0 \\
0 & 0 & 0 & 0 & 0 & 0 \\
0 & 1 & 0 & 0 & 0 & 0 \\
0 & 0 & 0 & 0 & 0 & 0 \\
0 & 0 & 0 & 0 & 0 & 0 \\
0 & 0 & 0 & 0 & 0 & 0
\end{array}\right) \\
\text { e } f_{1}^{* 1}(, \mathbb{Q})=\left(\begin{array}{cccccc}
0 & 0 & 0 & 0 & 0 & 0 \\
0 & -n & 0 & 0 & 0 & 0 \\
0 & 0 & 0 & 0 & 0 & 0 \\
0 & 0 & 0 & 0 & 0 & 0 \\
0 & 0 & 0 & 0 & 0 & 0 \\
0 & 0 & 0 & 0 & 0 & 0
\end{array}\right)
\end{aligned}
$$

usando o Teorema 2.27, página 42 , temos $L\left(f_{1}, f_{2}\right)=n$.

Caso $3(g \geq 1$ e $h>2$ ) Esse caso é uma generalização do caso anterior, podemos tomar:

\begin{tabular}{lclllll} 
& $f_{1 \#}$ & & \multicolumn{3}{c}{$f_{2 \#}$} \\
$a_{1}$ & $\mapsto$ & 1 & & $a_{1}$ & $\mapsto$ & $a_{1}$ \\
$b_{1}$ & $\mapsto$ & $b_{1}^{-n}$ & & $b_{1}$ & $\mapsto$ & 1 \\
$a_{2}$ & $\mapsto$ & 1 & & $a_{2}$ & $\mapsto$ & $b_{1}$ \\
$b_{2}$ & $\mapsto$ & 1 & & $b_{2}$ & $\mapsto$ & 1 \\
$\vdots$ & $\vdots$ & $\vdots$ & & $\vdots$ & $\vdots$ & $\vdots$ \\
$a_{h}$ & $\mapsto$ & 1 & & $a_{h}$ & $\mapsto$ & 1 \\
$b_{h}$ & $\mapsto$ & 1 & & $b_{h}$ & $\mapsto$ & 1 \\
$\alpha$ & $\mapsto$ & 1 & & $\alpha$ & $\mapsto$ & 1 \\
$\beta$ & $\mapsto$ & 1. & & $\beta$ & $\mapsto$ & $\beta$
\end{tabular}


O mesmo raciocínio usado no Caso 2 mostra que existe uma única classe de Nielsen no par $\left(f_{1}, f_{2}\right)$ com índice $n$.

Notemos que nos 3 casos $\left|f_{i \#}\left(\pi_{1}\left(K_{h}\right)\right): \pi_{1}\left(K_{g}\right)\right|=\infty$, assim em todos os casos $\operatorname{deg}\left(f_{i}\right)=0$.

Teorema 4.6. $B_{K}\left(g, h, d_{1}, d_{2}\right)=\infty$ para $h \geq g \geq 1,1 \leq d_{1} \leq \frac{h}{g}-1$ e $1 \leq d_{2}<$ $\frac{h}{g}-1$.

Demonstração: Denotando:

$$
\pi_{1}\left(K_{n}\right)=<\gamma_{1}, \gamma_{2}, \cdots, \gamma_{2 n+2} \mid \prod_{i=1}^{2 n+2} \gamma_{i}^{2}>
$$

definimos $f_{1 \#}: \pi_{1}\left(K_{h}\right) \mapsto \pi_{1}\left(K_{g}\right)$ : 
Se $d_{1} \neq 1$ :

$f_{1 \#}$

$\mapsto \quad 1$

$\begin{array}{lll}\gamma_{2} & \mapsto \gamma_{1} \\ \gamma_{3} & \mapsto \gamma_{2}\end{array}$

$\vdots \quad \vdots \quad \vdots$

$\gamma_{2 g+1} \quad \mapsto \gamma_{2 g}$

$\gamma_{2 g+2} \quad \mapsto \gamma_{2 g+1}$

$\gamma_{2 g+3} \quad: \quad \mapsto \quad \gamma_{2 g+1}^{-1}\left(\gamma_{2 g+1} \gamma_{2 g+2}\right)^{d_{1}}$

$\gamma_{2 g+4} \quad \mapsto\left(\gamma_{2 g+1} \gamma_{2 g+2}\right)^{-\left(d_{1}-1\right)} \gamma_{1}\left(\gamma_{2 g+1} \gamma_{2 g+2}\right)^{d_{1}-1}$

$\gamma_{2 g+5} \quad \mapsto \quad\left(\gamma_{2 g+1} \gamma_{2 g+2}\right)^{-\left(d_{1}-1\right)} \gamma_{2}\left(\gamma_{2 g+1} \gamma_{2 g+2}\right)^{d_{1}-1}$

$\vdots \quad \vdots \quad \vdots$

$\gamma_{4 g+3} \quad \mapsto \quad\left(\gamma_{2 g+1} \gamma_{2 g+2}\right)^{-\left(d_{1}-1\right)} \gamma_{2 g}\left(\gamma_{2 g+1} \gamma_{2 g+2}\right)^{d_{1}-1}$

$\gamma_{4 g+4} \quad \mapsto \quad\left(\gamma_{2 g+1} \gamma_{2 g+2}\right)^{-\left(d_{1}-2\right)} \gamma_{1}\left(\gamma_{2 g+1} \gamma_{2 g+2}\right)^{d_{1}-2}$

$\vdots \quad \vdots \quad \vdots$

$\gamma_{6 g+3} \quad \mapsto \quad\left(\gamma_{2 g+1} \gamma_{2 g+2}\right)^{-\left(d_{1}-2\right)} \gamma_{2 g}\left(\gamma_{2 g+1} \gamma_{2 g+2}\right)^{d_{1}-2}$

$\gamma_{6 g+4} \quad \mapsto \quad\left(\gamma_{2 g+1} \gamma_{2 g+2}\right)^{-\left(d_{1}-3\right)} \gamma_{1}\left(\gamma_{2 g+1} \gamma_{2 g+2}\right)^{d_{1}-3}$

$\vdots \quad \vdots \quad \vdots$

$\vdots \quad \vdots \quad \vdots$

$\gamma_{\left(2 d_{1}-2\right) g+3} \mapsto\left(\gamma_{2 g+1} \gamma_{2 g+2}\right)^{-2} \gamma_{2 g}\left(\gamma_{2 g+1} \gamma_{2 g+2}\right)^{2}$

$\gamma_{\left(2 d_{1}-2\right) g+4} \mapsto\left(\gamma_{2 g+1} \gamma_{2 g+2}\right)^{-1} \gamma_{1}\left(\gamma_{2 g+1} \gamma_{2 g+2}\right)$

$\vdots \quad \vdots \quad \vdots$

$\gamma_{2 d_{1} g+3} \quad \mapsto \quad\left(\gamma_{2 g+1} \gamma_{2 g+2}\right)^{-1} \gamma_{2 g}^{1-k}\left(\gamma_{2 g+1} \gamma_{2 g+2}\right)$

$\gamma_{2 d_{1} g+4} \mapsto 1$

$\gamma_{2 d_{1} g+5} \quad \mapsto \quad 1$

$\vdots \quad \vdots \quad \vdots$

$\gamma_{2 h-1} \quad \mapsto \quad 1$

$\gamma_{2 h} \quad \mapsto \quad 1$

$\gamma_{2 h+1} \quad \mapsto \quad 1$

$\gamma_{2 h+2} \quad \mapsto \quad\left(\gamma_{2 g+1} \gamma_{2 g+2}\right)^{-1} \gamma_{2 g}^{k}\left(\gamma_{2 g+1} \gamma_{2 g+2}\right)$

Que satisfaz:

$$
\begin{aligned}
f_{1 \#}\left(\gamma_{2 g+2}^{2} \gamma_{2 g+3}^{2}\right) & =\gamma_{2 g+1}^{2} \gamma_{2 g+1}^{-1}\left(\gamma_{2 g+1} \gamma_{2 g+2}\right)^{d_{1}} \gamma_{2 g+1}^{-1}\left(\gamma_{2 g+1} \gamma_{2 g+2}\right)^{d_{1}} \\
& =\prod_{i=0}^{d_{1}-1}\left(\gamma_{2 g+1} \gamma_{2 g+2}\right)^{-i} \gamma_{2 g+1}^{2} \gamma_{2 g+2}^{2}\left(\gamma_{2 g+1} \gamma_{2 g+2}\right)^{i}
\end{aligned}
$$


Se $d_{1}=1$ :

$\begin{array}{lll} & f_{1 \#} \\ \gamma_{1} & \mapsto & 1 \\ \gamma_{2} & \mapsto & \gamma_{3} \\ \gamma_{3} & \mapsto & \gamma_{4} \\ \vdots & \vdots & \vdots \\ \gamma_{2 g} & \mapsto & \gamma_{2 g+1} \\ \gamma_{2 g+1} & \mapsto & \gamma_{2 g+2} \\ \gamma_{2 g+2} & \mapsto & \gamma_{1} \\ \gamma_{2 g+3} & \mapsto & \gamma_{2}^{1-k} \\ \gamma_{2 g+4} & \mapsto & 1 \\ \gamma_{2 g+5} & \mapsto & 1 \\ \vdots & \vdots & \vdots \\ \gamma_{2 h} & \mapsto & 1 \\ \gamma_{2 h+1} & \mapsto & 1 \\ \gamma_{2 h+2} & \mapsto & \gamma_{2}^{k}\end{array}$

Definimos também:

Se $d_{2} \neq 1$ : 
$f_{2 \#}$

$\begin{array}{lll}\gamma_{1} & \mapsto & \gamma_{1} \\ \gamma_{2} & \mapsto & \gamma_{2} \\ \vdots & \vdots & \vdots \\ \gamma_{2 g+1} & \mapsto & \gamma_{2 g+1} \\ \gamma_{2 g+2} & \mapsto & \gamma_{2 g+2} \\ \gamma_{2 g+3} & \mapsto & \gamma_{1} \\ \gamma_{2 g+4} & \mapsto & \gamma_{2} \\ \vdots & \vdots & \vdots \\ \gamma_{4 g+2} & \mapsto & \gamma_{2 g} \\ \gamma_{4 g+3} & \mapsto & \left(\gamma_{2 g+1} \gamma_{2 g+2}\right)^{-1} \gamma_{1}\left(\gamma_{2 g+1} \gamma_{2 g+2}\right) \\ \vdots & \vdots & \vdots \\ \gamma_{6 g+2} & \mapsto & \left(\gamma_{2 g+1} \gamma_{2 g+2}\right)^{-1} \gamma_{2 g}\left(\gamma_{2 g+1} \gamma_{2 g+2}\right) \\ \gamma_{6 g+3} & \mapsto & \left(\gamma_{2 g+1} \gamma_{2 g+2}\right)^{-2} \gamma_{1}\left(\gamma_{2 g+1} \gamma_{2 g+2}\right)^{2} \\ \vdots & \vdots & \vdots \\ \vdots & \vdots & \vdots \\ \gamma_{\left(2 d_{2}-2\right) g+2} & \mapsto & \left(\gamma_{2 g+1} \gamma_{2 g+2}\right)^{-\left(d_{2}-3\right)} \gamma_{2 g}\left(\gamma_{2 g+1} \gamma_{2 g+2}\right)^{d_{2}-3} \\ \gamma_{\left(2 d_{2}-2\right) g+3} & \mapsto & \left(\gamma_{2 g+1} \gamma_{2 g+2}\right)^{-\left(d_{2}-2\right)} \gamma_{1}\left(\gamma_{2 g+1} \gamma_{2 g+2}\right)^{d_{2}-2} \\ \vdots & \vdots & \vdots \\ \gamma_{2 d_{2} g+2} & \mapsto & \left(\gamma_{2 g+1} \gamma_{2 g+2}\right)^{-\left(d_{2}-2\right)} \gamma_{2 g}\left(\gamma_{2 g+1} \gamma_{2 g+2}\right)^{d_{2}-2} \\ \gamma_{2 d_{2} g+3} & \mapsto & \gamma_{1} \\ \gamma_{2 d_{2} g+4} & \mapsto & \gamma_{1}^{-1} \\ \vdots & \vdots & \vdots \\ \gamma_{2 h-1} & \mapsto & \gamma_{1} \\ \gamma_{2 h} & \mapsto & \gamma_{1}^{-1} \\ \gamma_{2 h+1} & \mapsto & \left(\gamma_{2 g+1} \gamma_{2 g+2}\right)^{d_{2}-1} \gamma_{2 g+2}^{-1} \\ \gamma_{2 h+2} & \mapsto & \gamma_{2 g+2} \\ & & \end{array}$

Note que:

$$
\begin{aligned}
f_{2 \#}\left(\gamma_{2 h+1}^{2} \gamma_{2 h+2}^{2}\right) & =\left(\gamma_{2 g+1} \gamma_{2 g+2}\right)^{d_{2}-1} \gamma_{2 g+2}^{-1}\left(\gamma_{2 g+1} \gamma_{2 g+2}\right)^{d_{2}-1} \gamma_{2 g+2}^{-1} \gamma_{2 g+2}^{2} \\
& =\prod_{i=d_{2}-2}^{0}\left(\gamma_{2 g+1} \gamma_{2 g+2}\right)^{i} \gamma_{2 g+1}^{2} \gamma_{2 g+2}^{2}\left(\gamma_{2 g+1} \gamma_{2 g+2}\right)^{-i}
\end{aligned}
$$


Se $d_{2}=1$ :

\begin{tabular}{lll}
\multicolumn{3}{c}{$f_{2 \#}$} \\
$\gamma_{1}$ & $\mapsto$ & $\gamma_{1}$ \\
$\gamma_{2}$ & $\mapsto$ & $\gamma_{2}$ \\
$\vdots$ & $\vdots$ & $\vdots$ \\
$\gamma_{2 g+1}$ & $\mapsto$ & $\gamma_{2 g+1}$ \\
$\gamma_{2 g+2}$ & $\mapsto$ & $\gamma_{2 g+2}$ \\
$\gamma_{2 g+3}$ & $\mapsto$ & $\gamma_{1}$ \\
$\gamma_{2 g+4}$ & $\mapsto$ & $\gamma_{1}^{-1}$ \\
$\vdots$ & $\vdots$ & $\vdots$ \\
$\gamma_{2 h+1}$ & $\mapsto$ & $\gamma_{1}$ \\
$\gamma_{2 h+2}$ & $\mapsto$ & $\gamma_{1}^{-1}$
\end{tabular}

Assim temos que $f_{i \#}\left(\prod_{j=1}^{2 h+2} \gamma_{i}^{2}\right)=1$ para $i=1$, 2. o que garante que $f_{i \#}$ são homomorfismos e podem ser extendidos para aplicações $f_{i}: K_{h} \mapsto K_{g}$. Por construção $\operatorname{deg}\left(f_{1}\right)=d_{1}, \operatorname{deg}\left(f_{2}\right)=d_{2}$ e $f_{2}$ é fiel à orientação.

Notemos ainda que se $w \in \pi_{1}\left(K_{h}\right)$ e pode ser escrita como uma palavra em $<\gamma_{1}, \gamma_{2}, \cdots, \gamma_{t}>$ com $t \leq 2 g+2$ então $f_{2 \#}(w)=w$ e $f_{1 \#}(w)$ pode ser escrita como uma palavra em $\left\langle\gamma_{1}, \gamma_{2}, \cdots, \gamma_{t-1}>\right.$.

Assim se $w \in \pi_{1}\left(K_{g}\right)$, $w$ é uma palavra em $\left\langle\gamma_{1}, \gamma_{2}, \cdots, \gamma_{2 g+2}\right\rangle$, tomando-se $w^{\prime} \in \pi_{1}\left(K_{h}\right)$ a mesma palavra temos que:

$$
w \sim f_{1 \#} w^{\prime-1} \cdot w \cdot f_{2 \#} w^{\prime-1} \sim f_{1 \#} w^{\prime-1}
$$

que pode ser escrita como uma palavra em $\left\langle\gamma_{1}, \gamma_{2}, \cdots, \gamma_{2 g}\right\rangle$, repetindo-se o mesmo argumento, no máximo, $2 g$ vezes temos que $w \sim 1$ o que mostra que existe uma única classe de Reidemeister para o par $\left(f_{1}, f_{2}\right)$. Mais uma vez usaremos que o índice dessa classe é dado por $L\left(f_{1}, f_{2}\right)$, mais ainda, vamos mostrar que alterandose o parâmetro $k$ usado na definição de $f_{1 \#}$ podemos obter $L\left(f_{1}, f_{2}\right)$ tão grande quanto se queira.

Para o cálculo de $L\left(f_{1}, f_{2}\right)$ precisamos descrever como tais homomorfismos se comportam em relação a conjuntos geradores de $H_{1}\left(K_{h}, \mathbb{Z}\right)$ e $H_{1}\left(K_{g}, \mathbb{Z}\right)$ com as propriedades descritas na demostração da Proposição 2.2 de [Gonçalves \& Oliveira, página 160].

Tomando-se o seguinte sistema de geradores de $H_{1}\left(K_{n}, \mathbb{Z}\right)$ :

$$
\left\{\gamma_{1} \gamma_{2}, \gamma_{2}^{-1} \gamma_{2 n+2}, \gamma_{3} \gamma_{4}, \gamma_{4}^{-1} \gamma_{2 g+2}, \cdots, \gamma_{2 n}^{-1} \gamma_{2 n+2}, \gamma_{1}^{-1} \gamma_{2}^{-1} \cdots \gamma_{2 n}^{-1} \gamma_{2 n+1} \gamma_{2 n+2}, \gamma_{2 n+2}^{-1}\right\}
$$


podemos descrever $f_{1}: H_{1}\left(K_{h}\right) \mapsto H_{1}\left(K_{g}\right)$ matricialmente como a transposta do produto $T^{-1} \cdot M_{1} \cdot T$ onde $M_{1}$ no caso $d_{1} \neq 1$ é a matriz $(2 g+2) \times(2 h+2)$ :

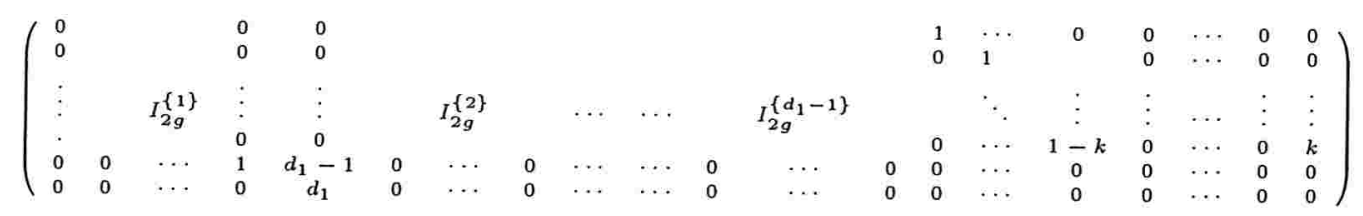

e $T$ é a matriz quadrada de ordem $2 h$ ou $2 g$ :

$$
\left(\begin{array}{ccccccccc}
1 & 0 & 0 & 0 & \cdots & 0 & 0 & 1 & 0 \\
1 & -1 & 0 & 0 & \cdots & 0 & 0 & 0 & 0 \\
0 & 0 & 1 & 0 & \cdots & 0 & 0 & 1 & 0 \\
0 & 0 & 1 & -1 & \cdots & 0 & 0 & 0 & 0 \\
\vdots & \vdots & \vdots & \vdots & \ddots & \vdots & \vdots & \vdots & \vdots \\
0 & 0 & 0 & 0 & \cdots & 1 & 0 & 1 & 0 \\
0 & 0 & 0 & 0 & \cdots & 1 & -1 & 0 & 0 \\
0 & 0 & 0 & 0 & \cdots & 0 & 0 & 1 & 0 \\
1 & -1 & 1 & -1 & \cdots & 1 & -1 & 1 & -1
\end{array}\right) .
$$

Dessa maneira obtemos, para $d_{1} \neq 1$ (na notação de [Gonçalves \& Oliveira]): 


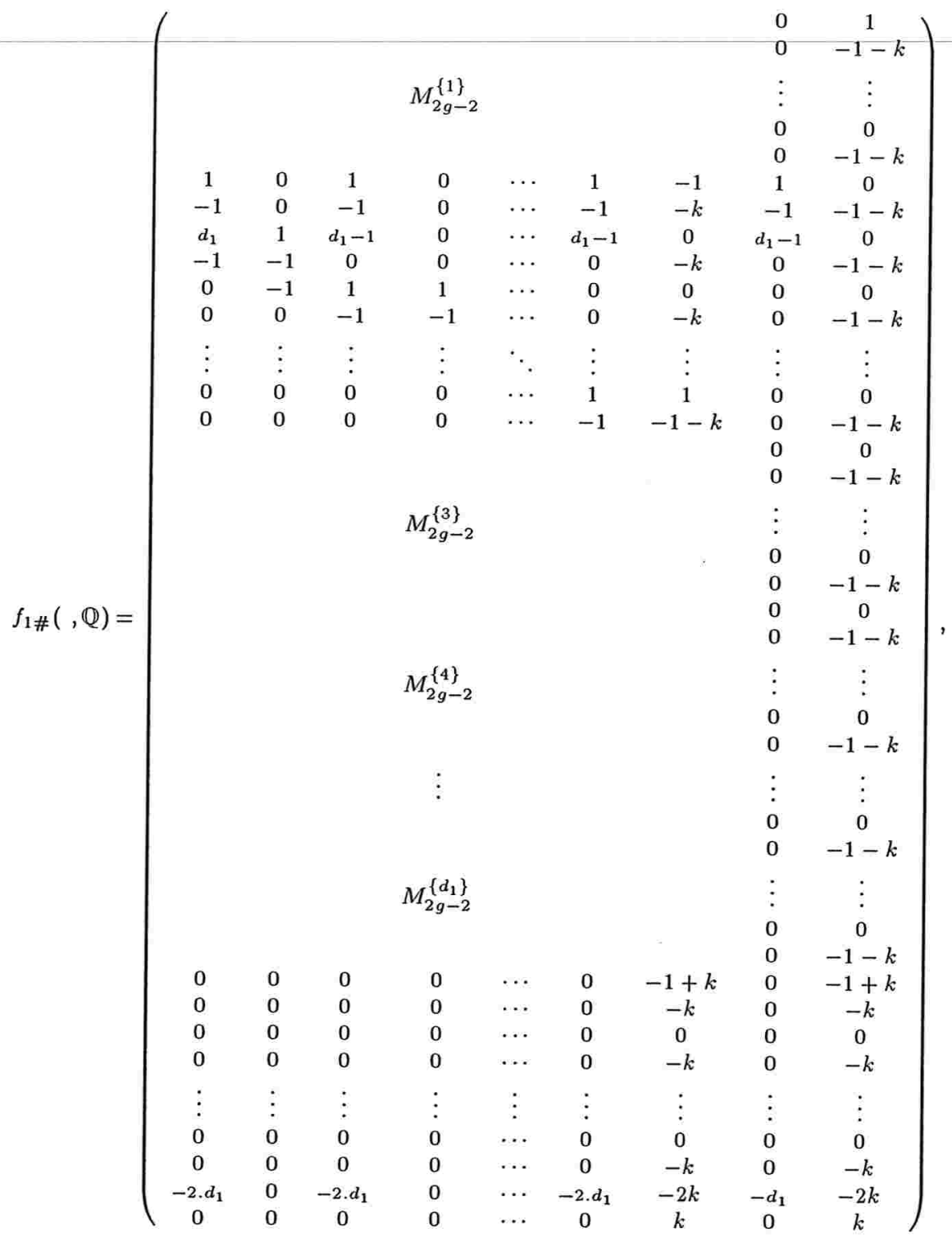

onde:

$$
M_{2 g-2}=\left(\begin{array}{ccccccc}
1 & 1 & 0 & 0 & \cdots & 0 & 0 \\
-1 & -1 & 0 & 0 & \cdots & 0 & -k \\
0 & -1 & 1 & 1 & \cdots & 0 & 0 \\
0 & 0 & -1 & -1 & \cdots & 0 & -k \\
\vdots & \vdots & \vdots & \vdots & \ddots & \vdots & \vdots \\
0 & 0 & 0 & 0 & \cdots & 1 & 1 \\
0 & 0 & 0 & 0 & \cdots & -1 & -1-k
\end{array}\right)
$$


Para $d_{1}=1$ :

$$
f_{1 \#}(, \mathbb{Q})=\left(\begin{array}{ccccccccccc}
0 & 0 & 1 & 1 & 0 & 0 & \cdots & 0 & 0 & 0 & 1 \\
0 & -k & -1 & -1 & 0 & 0 & \cdots & 0 & 0 & 0 & -1-k \\
0 & 0 & 0 & -1 & 1 & 1 & \cdots & 0 & 0 & 0 & 0 \\
0 & -k & 0 & 0 & -1 & -1 & \cdots & 0 & 0 & 0 & -1-k \\
\vdots & \vdots & \vdots & \vdots & \ddots & \vdots & \ddots & \vdots & \vdots & \vdots & \vdots \\
0 & 0 & 0 & 0 & 0 & 0 & \cdots & 1 & 1 & 0 & 0 \\
0 & -k & 0 & 0 & 0 & 0 & \cdots & -1 & -1 & 0 & -1-k \\
1 & 0 & 1 & 0 & 1 & 0 & \cdots & 1 & -1 & 1 & 0 \\
-1 & -k & -1 & 0 & -1 & 0 & \cdots & -1 & 0 & 0 & -1-k \\
1 & 1 & 0 & 0 & 0 & 0 & \cdots & 0 & 0 & 0 & 0 \\
1 & -1-k & 0 & -1 & 0 & 0 & \cdots & 0 & 0 & 0 & -1-k \\
0 & -1+k & 0 & 0 & 0 & 0 & \cdots & 0 & 0 & 0 & -1+k \\
0 & -k & 0 & 0 & 0 & 0 & \cdots & 0 & 0 & 0 & -1-k \\
0 & 0 & 0 & 0 & 0 & 0 & \cdots & 0 & 0 & 0 & 0 \\
0 & -k & 0 & 0 & 0 & 0 & \cdots & 0 & 0 & 0 & -1-k \\
\vdots & \vdots & \vdots & \vdots & \vdots & \vdots & \vdots & \vdots & \vdots & \vdots & \vdots \\
0 & 0 & 0 & 0 & 0 & 0 & \cdots & 0 & 0 & 0 & 0 \\
0 & -k & 0 & 0 & 0 & 0 & \cdots & 0 & 0 & 0 & -1-k \\
-2 & -2 k & -2 & 0 & -2 & 0 & \cdots & -2 & 0 & -1 & -2 k \\
0 & k & 0 & 0 & 0 & 0 & \cdots & 0 & 0 & 0 & k
\end{array}\right),
$$

De maneira análoga $f_{2}: H_{1}\left(K_{h}\right) \mapsto H_{1}\left(K_{g}\right)$ é descrita pela transposta do produto $T^{-1} \cdot M_{2} \cdot T$ onde $M_{2}$ é a matriz, no caso $d_{2} \neq 1,(2 g+2) \times(2 h+2)$ :

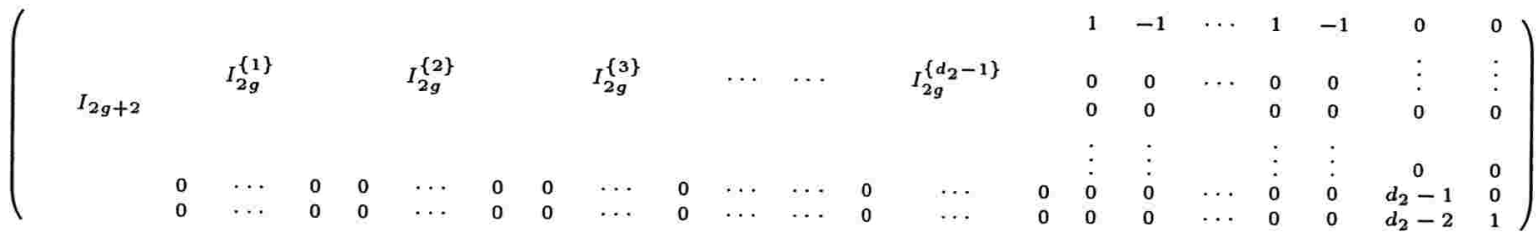

e no caso $d_{2}=1, M_{2}$ é a matriz $(2 g+2) \times(2 h+2)$ :

$$
\left(\begin{array}{cccc} 
& 0 & \cdots & 0 \\
& 0 & \cdots & 0 \\
I_{2 g+2} & \vdots & & \vdots \\
& 0 & \cdots & 0 \\
& 0 & \cdots & 0
\end{array}\right)
$$

Obtemos assim que, para $d_{2} \neq 1$ : 


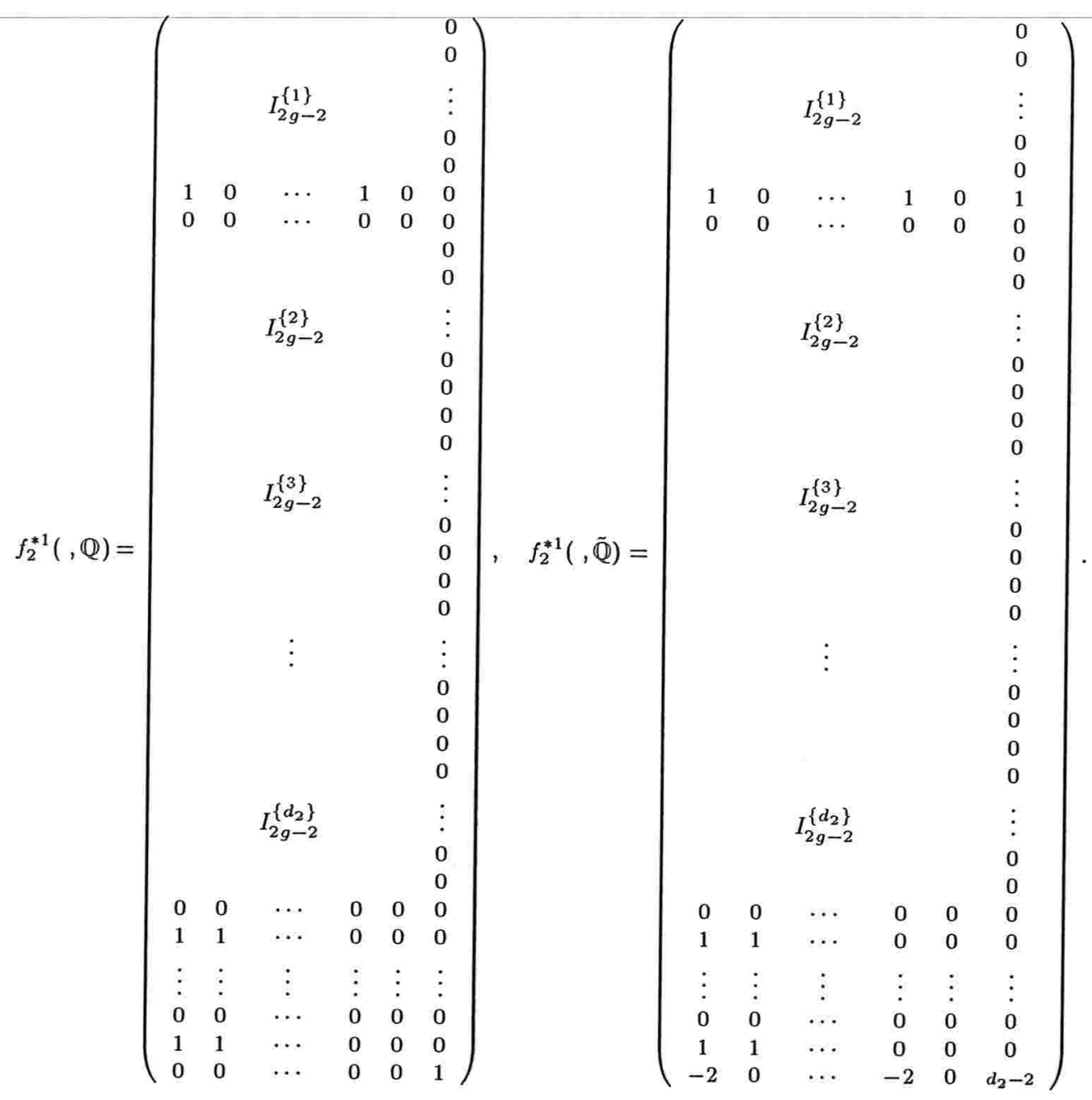

Para $d_{2}=1$ teremos:

$$
f_{2}^{* 1}(, \mathbb{Q})=\left(\begin{array}{ccccc}
1 & 0 & \cdots & 0 & 0 \\
-1 & 0 & \cdots & 0 & 0 \\
0 & 0 & & & 0 \\
-1 & -1 & & & . \\
\vdots & \vdots & I_{2 g-4} & & \vdots \\
0 & 0 & & & . \\
-1 & -1 & & & 0 \\
1 & 0 & \cdots & 0 & 0 \\
-1 & -1 & \cdots & 0 & 0 \\
0 & 0 & \cdots & 0 & 0 \\
0 & 0 & \cdots & 0 & 0 \\
\vdots & \vdots & \cdots & \vdots & \vdots \\
0 & 0 & \cdots & 0 & 0 \\
1 & 1 & \cdots & 0 & 1
\end{array}\right), \quad f_{2}^{* 1}(, \tilde{\mathbb{Q}})=\left(\begin{array}{ccccc}
1 & 0 & \cdots & 0 & 0 \\
-1 & 0 & \cdots & 0 & 1 \\
0 & 0 & & & 0 \\
-1 & -1 & & & . \\
\vdots & \vdots & I_{2 g-4} & & \vdots \\
0 & 0 & & & . \\
-1 & -1 & & & 0 \\
1 & 0 & \cdots & 1 & 0 \\
-1 & -1 & \cdots & 0 & 0 \\
0 & 0 & \cdots & 0 & 0 \\
0 & 0 & \cdots & 0 & 0 \\
\vdots & \vdots & \cdots & \vdots & \vdots \\
0 & 0 & \cdots & 0 & 0 \\
-2 & 0 & \cdots & -1 & 0
\end{array}\right) .
$$


Obtemos então, por cálculos diretos usando [Gonçalves \& Oliveira, Teorema 2.8, página 165$]$ :

$$
L\left(f_{1}, f_{2}\right)=\left\{\begin{array}{clc}
-3+4 k & \text { se } & d_{2}=1, d_{1}=1, g \geq 2 ; \\
-2+4 k & \text { se } & d_{2}=1, d_{1}>1, g \geq 2 ; \\
d_{2}-1+4 k & \text { se } & d_{2}>1, d_{1}=1, g \geq 2 ; \\
-2\left(d_{2}-d_{1}\right)-1+4 k & \text { se } & d_{1} \geq d_{2}-1>1, g=2, d_{1} \neq h-2 ; \\
d_{2}-d_{1}+4 k & \text { se } & d_{2} \geq d_{1}>1, g \geq 3 ; \\
2\left(d_{2}-d_{1}\right)+4 k & \text { se } & \left(d_{1} \geq d_{2}>1, g \geq 3\right) \text { ou }\left(g=2, \quad d_{1}=h-2\right)
\end{array}\right.
$$

\subsection{Aplicações de $K_{h} \mapsto P_{g}$}

Definimos agora:

$$
B_{K P}\left(g, h, d_{1}, d_{2}\right)=\sup \left\{\left|\operatorname{ind}\left(f_{1}, f_{2}, C\right)\right| \mid \operatorname{deg}\left(f_{1}\right)=d_{1}, \operatorname{deg}\left(f_{2}\right)=d_{2}\right\},
$$

onde o supremo é tomado para todas as classes de coincidência $C$ de todos os pares de aplicações ${ }^{8}\left(f_{1}, f_{2}\right): K_{h} \mapsto P_{g}$ com tais graus e $f_{2}$ fiel à orientação.

Teorema 4.7. $B_{K P}(g, h, 0,0)=\infty$ se $h \geq 2$ e $g \geq 1$.

Demonstração: Novamente definiremos um par de homomorfismos $f_{1 \#}, f_{2 \#}$ em $\pi_{1}$ que se extende como aplicações $\left(f_{1}, f_{2}\right)$ entre as superfícies requeridas (que são espaços de Eilenberg-MacLane do tipo $\left.K\left[\pi_{1}\right]\right)$.

Usando a notação anterior para $\pi_{1}\left(K_{h}\right)$ e denotando:

$$
\pi_{1}\left(P_{g}\right)=<a_{1}, b_{1}, a_{2}, b_{2}, \ldots, a_{g}, b_{g}, \gamma \mid \prod_{i=1}^{g}\left[a_{i}, b_{i}\right] \cdot \gamma^{2}>,
$$

\begin{tabular}{|c|c|c|c|c|c|}
\hline \multicolumn{3}{|c|}{$f_{1 \#}$} & \multicolumn{3}{|c|}{$f_{2 \#}$} \\
\hline$a_{1}$ & $\mapsto$ & 1 & $a_{1}$ & $\mapsto$ & $a_{1}$ \\
\hline$b_{1}$ & $\mapsto$ & $b_{1}^{-n}$ & $b_{1}$ & $\mapsto$ & 1 \\
\hline$a_{2}$ & $\mapsto$ & 1 & $a_{2}$ & $\mapsto$ & $b_{1}$ \\
\hline$b_{2}$ & $\mapsto$ & 1 & $b_{2}$ & $\mapsto$ & 1 \\
\hline$\alpha$ & $\mapsto$ & 1 & $\alpha$ & $\mapsto$ & 1 \\
\hline$\beta$ & $\mapsto$ & 1. & $\beta$ & $\mapsto$ & $\gamma$ \\
\hline
\end{tabular}

tomamos $f_{1 \#}, f_{2 \#}: \pi_{1}\left(K_{2}\right) \mapsto \pi_{1}\left(P_{1}\right)$ dados por:

${ }^{8}$ Para as definições de $K_{h}$ e $P_{g}$ ver a seção 2.5 na página 40. 
Dessa forma teremos:

$$
\begin{aligned}
f_{2}^{* 1}(, \mathbb{Q}) & =\left(\begin{array}{ll}
1 & 0 \\
0 & 0 \\
0 & 1 \\
0 & 0 \\
0 & 0
\end{array}\right), f_{2}^{* 1}(, \tilde{\mathbb{Q}})=\left(\begin{array}{ll}
1 & 0 \\
0 & 0 \\
0 & 1 \\
0 & 0 \\
0 & 0
\end{array}\right) \\
& \text { e } f_{1}^{* 1}(, \mathbb{Q})=\left(\begin{array}{cc}
0 & 0 \\
0 & -n \\
0 & 0 \\
0 & 0 \\
0 & 0
\end{array}\right)
\end{aligned}
$$

$\mathrm{O}$ mesmo argumento usado para $f_{1}, f_{2}: K_{2} \mapsto K_{1}$ mostra que temos uma única classe de Nielsen com índice $n$ (para calcular $L\left(f_{1}, f_{2}\right)$ usamos o Teorema 2.28 da página 43).

Como foi feito no caso de $f_{1}, f_{2}: K_{2} \mapsto K_{1}$ esse exemplo pode ser extendido para $f_{1}, f_{2}: K_{h} \mapsto P_{g}$ com $h \geq 2$ e $g \geq 1$.

Para aplicações de grau não nulo devemos observar que segundo [GKZ, Teorema 2.5 (c)] se $f: K_{h} \mapsto P_{g}$ é fiel à orientação temos que:

$$
\chi\left(K_{h}\right) \equiv \operatorname{deg}(f) \chi\left(P_{g}\right) \bmod 2
$$

e portanto $\operatorname{deg}(f)$ é par. Como supomos $f_{2}$ fiel à orientação temos que $d_{2}$ será sempre par.

Teorema 4.8. $B_{K P}\left(g, h, d_{1}, d_{2}\right)=\infty$ se $h \geq 2, g \geq 1, d_{1}>0$ e $d_{2}>0$

Demonstração: Tomando-se as seguintes funções descritas no grupo fundamental ${ }^{9}$ das supefícies:

\footnotetext{
${ }^{9} \mathrm{Com}$ as mesmas notações usadas anteriormente.
} 


$$
\begin{array}{lll} 
& f_{1 \#} \\
\gamma_{1} & \mapsto & 1 \\
\gamma_{2} & \mapsto & \gamma_{1} \\
\gamma_{3} & \mapsto & \gamma_{2} \\
\vdots & \vdots & \vdots \\
\gamma_{2 g+1} & \mapsto & \gamma_{2 g} \\
\gamma_{2 g+2} & \mapsto & \gamma_{2 g}^{-1}\left(\gamma_{2 g} \gamma_{2 g+1}\right)^{d_{1}} \\
\gamma_{2 g+3} & \mapsto & \left(\gamma_{2 g} \gamma_{2 g+1}\right)^{-\left(d_{1}-1\right)} \gamma_{1}\left(\gamma_{2 g} \gamma_{2 g+1}\right)^{d_{1}-1} \\
\gamma_{2 g+4} & \mapsto & \left(\gamma_{2 g} \gamma_{2 g+1}\right)^{-\left(d_{1}-1\right)} \gamma_{2}\left(\gamma_{2 g} \gamma_{2 g+1}\right)^{d_{1}-1} \\
\vdots & \vdots & \vdots \\
\gamma_{4 g+1} & \mapsto & \left(\gamma_{2 g} \gamma_{2 g+1}\right)^{-\left(d_{1}-1\right)} \gamma_{2 g-1}\left(\gamma_{2 g} \gamma_{2 g+1}\right)^{d_{1}-1} \\
\gamma_{4 g+2} & \mapsto & \left(\gamma_{2 g} \gamma_{2 g+1}\right)^{-\left(d_{1}-2\right)} \gamma_{1}\left(\gamma_{2 g} \gamma_{2 g+1}\right)^{d_{1}-2} \\
\vdots & \vdots & \vdots \\
\vdots & \vdots & \vdots \\
\gamma_{\left(2 d_{1}-2\right) g+4} & \mapsto & \left(\gamma_{2 g} \gamma_{2 g+1}\right)^{-2} \gamma_{2 g-1}\left(\gamma_{2 g} \gamma_{2 g+1}\right)^{2} \\
\gamma_{\left(d_{1}-1\right)(2 g-1)+3} & \mapsto & \left(\gamma_{2 g} \gamma_{2 g+1}\right)^{-2} \gamma_{2 g-1}\left(\gamma_{2 g} \gamma_{2 g+1}\right)^{2} \\
\gamma_{\left(d_{1}-1\right)(2 g-1)+4} & \mapsto & \left(\gamma_{2 g} \gamma_{2 g+1}\right)^{-1} \gamma_{1}\left(\gamma_{2 g} \gamma_{2 g+1}\right) \\
\vdots & \vdots & \vdots \\
\gamma_{d_{1}(2 g-1)+3} & \mapsto & \left(\gamma_{2 g} \gamma_{2 g+1}\right)^{-1} \gamma_{2 g-1}^{1-k}\left(\gamma_{2 g} \gamma_{2 g+1}\right) \\
\gamma_{d_{1}(2 g-1)+4} & \mapsto & 1 \\
\gamma_{d_{1}(2 g-1)+5} & \mapsto & 1 \\
\vdots & \vdots & \vdots \\
\gamma_{2 h} & \mapsto & \left(\gamma_{2 g} \gamma_{2 g+1}\right)^{-1} \gamma_{2 g-1}^{k}\left(\gamma_{2 g} \gamma_{2 g+1}\right) \\
\gamma_{2 h+1} & \mapsto \\
\gamma_{2 h+2} & \mapsto &
\end{array}
$$$$
\gamma_{\left(d_{1}-1\right)(2 g-1)+3} \mapsto\left(\gamma_{2 g} \gamma_{2 g+1}\right)^{-2} \gamma_{2 g-1}\left(\gamma_{2 g} \gamma_{2 g+1}\right)^{2}
$$$$
\vdots \quad \vdots \quad \vdots
$$$$
\gamma_{d_{1}(2 g-1)+4} \mapsto 1
$$$$
\vdots \quad \vdots \quad \vdots
$$$$
\gamma_{2 h+1} \quad \mapsto 1
$$ 


$\begin{array}{lll} & f_{2 \#} \\ \gamma_{1} & \mapsto \gamma_{1} \\ \gamma_{2} & \mapsto \gamma_{2} \\ \vdots & \vdots & \vdots \\ \gamma_{2 g+1} & \mapsto & \gamma_{2 g+1} \\ \gamma_{2 g+2} & \mapsto & \gamma_{1} \\ \gamma_{2 g+3} & \mapsto & \gamma_{2} \\ \vdots & \vdots & \vdots \\ \gamma_{4 g} & \mapsto & \gamma_{2 g-1} \\ \gamma_{4 g+1} & \mapsto & \left(\gamma_{2 g} \gamma_{2 g+1}\right)^{-1} \gamma_{1}\left(\gamma_{2 g} \gamma_{2 g+1}\right) \\ \vdots & \vdots & \vdots \\ \gamma_{6 g-1} & \mapsto & \left(\gamma_{2 g} \gamma_{2 g+1}\right)^{-1} \gamma_{2 g-1}\left(\gamma_{2 g} \gamma_{2 g+1}\right) \\ \gamma_{6 g} & \mapsto & \left(\gamma_{2 g} \gamma_{2 g+1}\right)^{-2} \gamma_{1}\left(\gamma_{2 g} \gamma_{2 g+1}\right)^{2} \\ \vdots & \vdots & \vdots \\ \vdots & \vdots & \vdots \\ \gamma_{\left(d_{2}-1\right)(2 g-1)+2} & \mapsto & \left(\gamma_{2 g} \gamma_{2 g+1}\right)^{-\left(d_{2}-3\right)} \gamma_{2 g-1}\left(\gamma_{2 g} \gamma_{2 g+1}\right)^{d_{2}-3} \\ \gamma_{\left(d_{2}-1\right)(2 g-1)+3} & \mapsto & \left(\gamma_{2 g} \gamma_{2 g+1}\right)^{-\left(d_{2}-2\right)} \gamma_{1}\left(\gamma_{2 g} \gamma_{2 g+1}\right)^{d_{2}-2} \\ \vdots & \vdots & \vdots \\ \gamma_{d_{2}(2 g-1)+2} & \mapsto & \left(\gamma_{2 g} \gamma_{2 g+1}\right)^{-\left(d_{2}-2\right)} \gamma_{2 g-1}\left(\gamma_{2 g} \gamma_{2 g+1}\right)^{d_{2}-2} \\ \gamma_{d_{2}(2 g-1)+3} & \mapsto & \gamma_{1} \\ \gamma_{d_{2}(2 g-1)+4} & \mapsto & \gamma_{1}^{-1} \\ \vdots & \vdots & \vdots \\ \gamma_{2 h-1} & \mapsto & \gamma_{1} \\ \gamma_{2 h} & \mapsto & \left(\gamma_{2 g} \gamma_{2 g+1}\right)^{d_{2}-1} \gamma_{2 g+1}^{-1} \\ \gamma_{2 h+1} & \mapsto & \gamma_{2 g+1} \\ \gamma_{2 h+2} & & \end{array}$

Temos que ambas definem homomorfismos no grupo fundamental, portanto se estendem para aplicações $f_{1}, f_{2}: K_{h} \mapsto P_{g}$, além disso $f_{2}$ é fiel à orientação, $\operatorname{deg}\left(f_{1}\right)=d_{1}$ e $\operatorname{deg}\left(f_{2}\right)=d_{2}$.

Usando o mesmo raciocínio usado na demostração do Teorema 4.6 verificamos que o par $\left(f_{1}, f_{2}\right)$ tem somente uma classe de Reidemeister, cujo índice pode ser calculado pelo número de Lefshtez.

Considerando os seguintes conjuntos de geradores para $H_{1}\left(K_{h} ; \mathbb{Z}\right)$ e $H_{1}\left(P_{g} ; \mathbb{Z}\right)$ : $\left\{\gamma_{1} \gamma_{2}, \gamma_{2}^{-1} \gamma_{2 h+2}, \gamma_{3} \gamma_{4}, \gamma_{4}^{-1} \gamma_{2 h+2}, \cdots, \gamma_{2 h}^{-1} \gamma_{2 h+2}, \gamma_{1}^{-1} \gamma_{2}^{-1} \cdots \gamma_{2 h}^{-1} \gamma_{2 h+1} \gamma_{2 h+2}, \gamma_{2 h+2}^{-1}\right\}$ e

$$
\left\{\gamma_{1} \gamma_{2}, \gamma_{2}^{-1} \gamma_{2 g+1}, \gamma_{3} \gamma_{4}, \gamma_{4}^{-1} \gamma_{2 g+1}, \cdots, \gamma_{2 g}^{-1} \gamma_{2 g+1}, \gamma_{2 g+1}\right\}
$$


Podemos descrever ${ }^{10}$ os homomorfismos induzidos em cohomologia pela $f_{1}$ como:

$$
f_{1}^{* 1}(, \mathbb{Q})=\left(\begin{array}{ccccccc}
1 & 1 & 0 & 0 & \cdots & 0 & 0 \\
& & & L^{\{1\}} & & & \\
& & & & & & \\
0 & 0 & \cdots & 0 & 0 & 0 & -d_{1} \\
0 & 0 & \cdots & 0 & 0 & k & d_{1}-1+k \\
& & & J^{\{2\}} & & & \\
& & & & & & \\
& & & L^{\{3\}} & & & \\
& & & & & & \\
& & & J^{\{4\}} & & & \\
& & & & & & \\
& & & \vdots & & & \\
& & & L^{\left\{d_{1}-1\right\}} & & & \\
& & & & & & \\
& & & & & k & k \\
& & & & & \vdots & \vdots \\
& & I_{2 g-2} & & & \vdots & \vdots \\
0 & 0 & \cdots & 0 & 0 & 1-k & 1-k \\
0 & 0 & \cdots & 0 & 0 & k & k \\
0 & 0 & \cdots & 0 & 0 & 0 & 0 \\
\vdots & \vdots & \vdots & \vdots & \vdots & \vdots & \vdots \\
0 & 0 & \cdots & 0 & 0 & k & k \\
0 & 0 & \cdots & 0 & 0 & 0 & 0 \\
0 & 0 & \cdots & 0 & 0 & -k & -k
\end{array}\right) .
$$

no caso de $d_{1}$ ser par e

\footnotetext{
${ }^{10}$ Utilizando novamente as idéias da demonstração do Teorema 4.6
} 


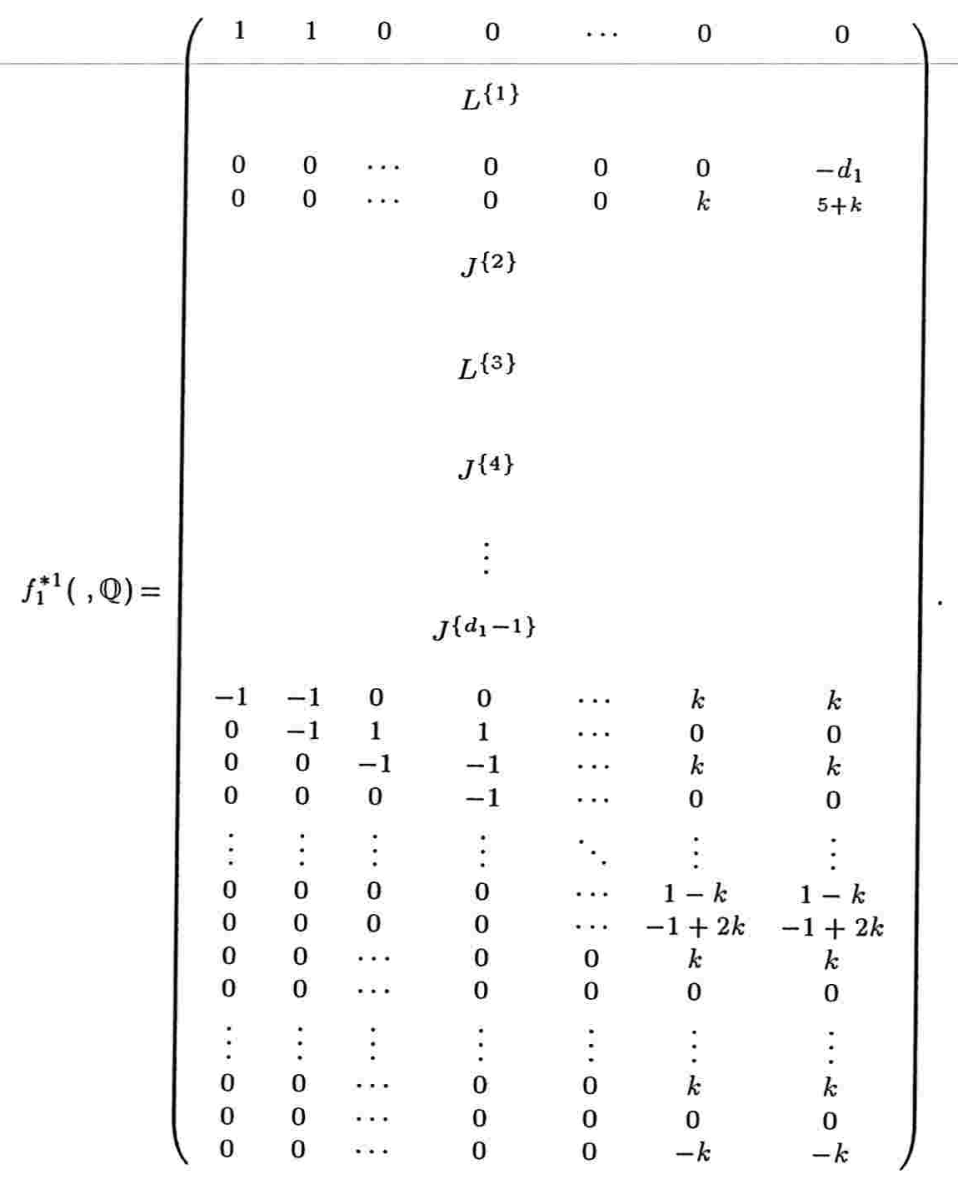

para $d_{1}$ ímpar.

Onde ${ }^{11}$ :

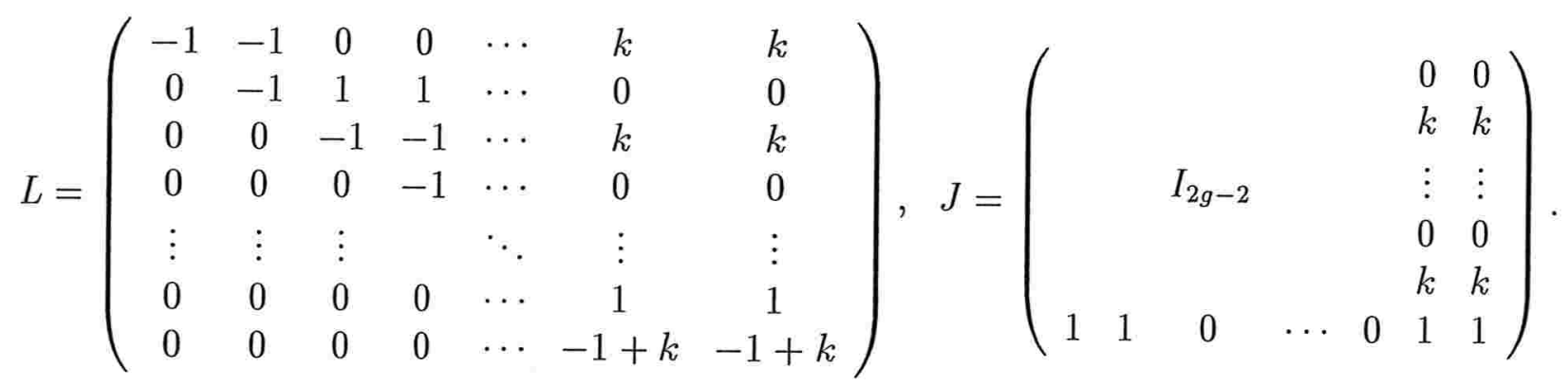

Bem como, para $f_{2}$ teremos:

\footnotetext{
${ }^{11}$ Tanto $L$ como $J$ são matrizes $(2 g-1) \times(2 g)$.
} 


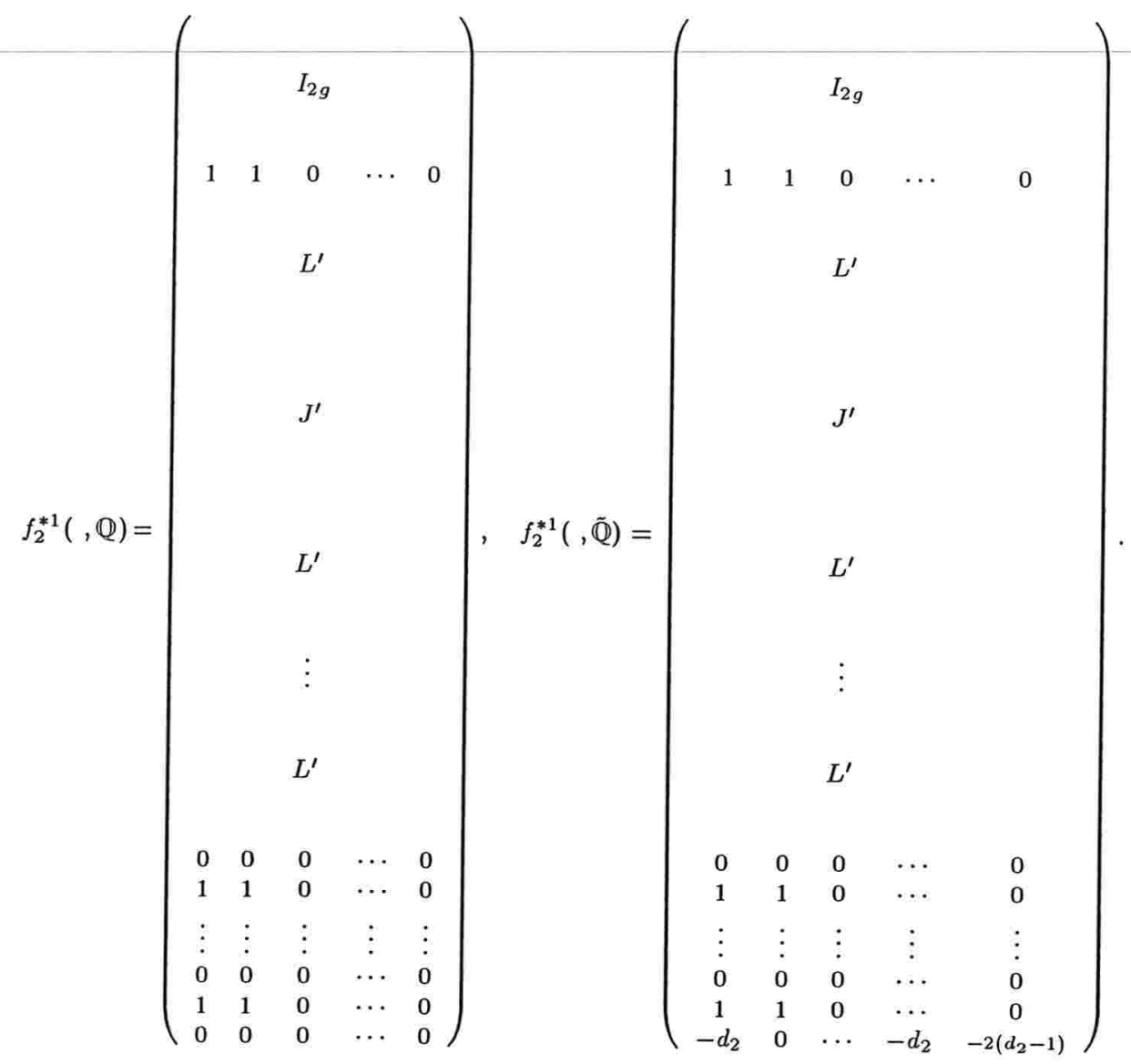

Onde:

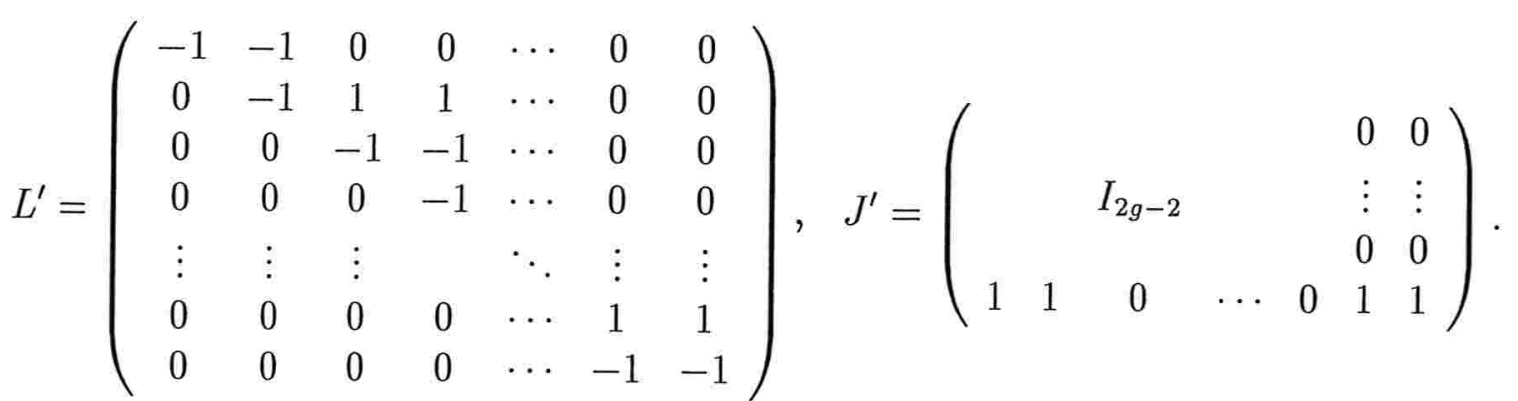

Agora, com cálculos diretos, usando [Gonçalves \& Oliveira, Teorema 2.7, página 164] obtemos $^{12}$ :

\footnotetext{
${ }^{12}$ Note que de acordo com a observação feita na página $72 d_{2}$ é sempre par, assim $L\left(f_{1}, f_{2}\right)$ será sempre inteiro
} 


$$
L\left(f_{1}, f_{2}\right)=\left\{\begin{array}{cccc}
\frac{3 d_{2}}{2}-d_{1}+\left(d_{2}-3\right) k & \text { se } & g \geq 2, & d_{1}>d_{2} \\
\frac{d_{2}}{2}+\left(d_{2}-3\right) k & \text { se } & g \geq 2, & d_{2} \geq d_{1} \\
\frac{5 d_{2}}{2}-d_{1}-3+\left(d_{2}-3\right) k & \text { se } & g=2, & d_{1}>d_{2} \\
\frac{d_{2}}{2}+d_{1}-3+\left(d_{2}-3\right) k & \text { se } & g=2, & d_{2} \geq d_{1}
\end{array}\right.
$$

\subsection{Outros Casos}

Estudamos até aqui os casos onde o domínio das aplicações é uma superfície do tipo $K_{n}$ e o contra-domínio é não orientável. Caso o domínio seja não orientável e o contra-domínio orientável as técnicas aqui apresentadas não podem ser utilizadas pois nesse caso a suposição de $f_{2}$ ser fiel à orientação não se realiza.

Para aplicações entre duas superfícies do tipo $P_{n}$ ou quando o domínio for uma superfície orientável resultados semelhantes podem ser obtidos usando-se as mesmas técnicas.

\subsection{Alguns Resultados com Limitação}

\subsubsection{Recobrimentos}

O resultado que apresentaremos nessa subseção é uma adaptação, que engloba o caso não orientável, de [Gonçalves \& Jiang, Proposição 11, página 86] onde uma das aplicações é um recobrimento. Aqui, com as mesmas hipóteses de [Gonçalves \& Jiang], obtemos um limitante para o índice de uma classe de coincidência entre superfícies orientáveis ou não. Lembramos que um recobrimento sempre é fiel a orientação.

Teorema 4.9. Sejam $S_{A}$ e $S_{B}$ duas superfíces compactas e sem bordo, $f: S_{A} \mapsto S_{B}$ uma aplicação contínua e $p: S_{A} \mapsto S_{B}$ um recobrimento. Se existe um subgrupo $K \subset \pi_{1}\left(S_{A}\right)$ tal que:

- $\left[\pi_{1}\left(S_{A}\right): K\right]=n<\infty$;

- $f_{\pi}(K) \subset p_{\pi}(K)$

então para toda classe de coincidência de Nielsen, $C$, do par $(f, p),|\operatorname{ind}(f, p, C)| \leq$ $\left|2 n \chi\left(S_{A}\right)-1\right|$. 
Demonstração: Nas condições acima $\operatorname{sejam}^{13} x_{0} \in C o i n(f, p) \subset S_{A}$ e $C$ a classe de coincidência que contem $x_{0}$.

Como $\left[\pi_{1}\left(S_{A}\right): K\right]=n<\infty$; existe uma superfície compacta sem bordo $S_{K}$ e uma aplicação de recobrimento, $q:\left(S_{K}, x_{0}^{\prime}\right) \mapsto\left(S_{A}, x_{0}\right)$, de ordem $n$. Notemos que $\chi\left(S_{K}\right)=n \chi\left(S_{A}\right)$ e $q_{\#}\left(\pi_{1}\left(S_{k}, x_{0}^{\prime}\right)\right)=K$.

Se $\overline{x_{0}}=f\left(x_{0}\right)=p\left(x_{0}\right)$, existe uma aplicação $f^{\prime}: S_{K} \mapsto S_{k}$ que torna o seguinte diagrama comutativo:

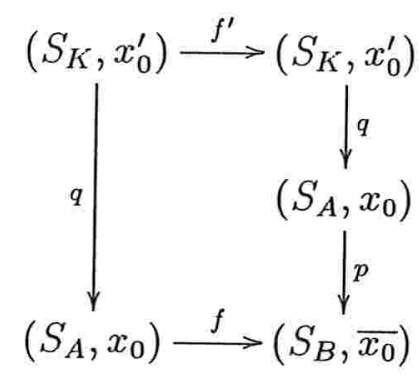
$x_{0}^{\prime}$.

Denotaremos por $C^{\prime}$ a classe de coincidência do par $\left(f^{\prime}, i d\right)$ que contém o ponto

Notemos que $C \operatorname{oin}\left(f^{\prime}, i d\right)$ se inclui naturalmente em $C \operatorname{oin}\left(p \circ q \circ f^{\prime}, p \circ q\right)$ e como $p \circ q$ é um recobrimento, pelo [Gonçalves \& Jiang, Lema 1(iv), páginas 75 e 76,], $C^{\prime}$ é uma classe de coincidência do par $\left(p \circ q \circ f^{\prime}, p \circ q\right)$. Assim, pela comutatividade do diagrama acima $C^{\prime}$ é uma classe de coincidência do par $(f \circ q, p \circ q)$. Naturalmente $\mid$ ind $\left(f^{\prime}, i d, C\right)|=| \operatorname{ind}\left(f \circ q, p \circ q, C^{\prime}\right) \mid$.

Como $q$ é um recobrimento, usando [Gonçalves \& Jiang, Lema 2(iii), página 76] concluímos que $C^{\prime}$ cobre $C, n^{\prime}$ vezes com $1 \leq n^{\prime} \leq n$. Usando [Dobreńko \& Jezierski, Lema 2.1, corolário 2.2 e Lema 2.3, páginas 77 e 78] e [Gonçalves \& Jezierski, Lema 5.3, página 20] obtemos que $\mid$ ind $\left(f \circ q, p \circ q, C^{\prime}\right)\left|=n^{\prime}\right| i n d(f, p, C) \mid$ também no caso não orientável ${ }^{14}$. Assim obtemos que $|\operatorname{ind}(f, p, C)| \leq \mid$ ind $\left(f \circ q, p \circ q, C^{\prime}\right) \mid=$ $\left|i n d\left(f^{\prime}, i d, C^{\prime}\right)\right|$.

Como $C^{\prime}$ é uma classe de coincidência do par $\left(f^{\prime}, i d\right), C^{\prime}$ pode ser vista como uma classe deponto fixo de $f^{\prime}: S_{k} \mapsto S_{K}$ e, por [Jiang 2, Teorema 2, página 477], $\left|\operatorname{ind}\left(f^{\prime}, i d, C^{\prime}\right)\right| \leq\left|2 \chi\left(S_{K}\right)-1\right|$; portanto $|\operatorname{ind}(f, p, C)| \leq\left|2 n \chi\left(S_{A}\right)-1\right|$.

\footnotetext{
${ }^{13}$ Caso Coin $(f, p)=\emptyset$ nada temos a demonstrar.

${ }^{14}$ Cabe lembrar aqui a equivalência do semi-índice definido em [Dobreńko \& Jezierski] e do valor absoluto do índice usado aqui (ver [Gonçalves \& Jezierski]).
} 


\subsection{2 $B_{K}(g, g, 0,1)$ e $B_{P}(g, g, 0,1)$}

De maneira análoga a definição de $B_{K}\left(g, h, d_{1}, d_{2}\right)$ feita na seção 4.1 na página 54 podemos definir: $B_{P}\left(g, h, d_{1}, d_{2}\right)$ para aplicações entre duas superfícies do tipo $P_{n}$. Podemos agora enunciar:

Teorema 4.10. $B_{K}(g, g, 0,1)=2 g-1$ e $B_{P}(g, g, 0,1)=2 g$ para $g \geq 1$.

Demonstração: Seja $M_{n}$ a superfície não orientável que admite como recobrimento duplo orientável a superfície $S_{n}$ (de genus $n$ ). Dado um par $f_{1}, f_{2}: M_{n} \mapsto$ $M_{n}$, tal que $f_{2}$ tem grau \pm 1 , sabemos que $f_{2}$ é homotópica a um homeomorfismo $f_{2}^{\prime}$ e que a inclusão $\operatorname{Coin}\left(f_{1}, f_{2}\right) \mapsto F i x\left(\left(f_{2}^{\prime}\right)^{-1} \circ f_{1}\right)$ preserva índices de classes de Nielsen, além disso $\left|\operatorname{deg}\left(f_{1}\right)\right|=\left|\operatorname{deg}\left(\left(f_{2}^{\prime}\right)^{-1} \circ f_{1}\right)\right|$. Assim podemos lidar com o par $\left(f_{1}, f_{2}\right)$ como sendo o par $\left(\left(f_{2}^{\prime}\right)^{-1} \circ f_{1}, i d\right)$ o que nos permite recorrer a definição e as propriedades do índice de classes de ponto fixo. Por simplicidade de notação denotaremos $\left(f_{2}^{\prime}\right)^{-1} \circ f_{1}$ como $f$.

- Primeiramente vamos mostrar que dada $C \subset F i x(f)$ uma classe de Nielsen então $|i n d(C)| \leq n$. Para tanto notamos que se $\operatorname{deg}(f)=0, f$ se fatora no 1-esqueleto de $M_{n}$ e por [Lyndon \& Schupp, Proposição 6.6, página 53] temos que $f_{\#}\left(\pi_{1}\left(M_{n}\right)\right)$ é um subgrupo livre de posto, no máximo ${ }^{15}, \frac{n+1}{2}$, assim podemos fatorar $f$ pela união por um ponto ${ }^{16}$ de, no máximo, $\frac{n+1}{2}$ cópias de $S^{1}$. Usando os resultados de [Jiang 2] e a propriedade comutativa do índice de classes de ponto fixo obtemos que $|i n d(C)| \leq n$.

- Construiremos agora um exemplo nas condições do Teorema com $\mid$ ind $(C) \mid=$ n. Para $n$ ímpar e considerando $f: \bigvee_{i}^{\frac{n+1}{2}} S^{1} \mapsto \bigvee_{i}^{\frac{n+1}{2}} S^{1}$, a aplicação que leva cada cópia de $S^{1}$ em si mesma com grau 2 deixando um único ponto fixo na intersecção das cópias de $S^{1}$, podemos tomar $\bigvee_{i}^{\frac{n+1}{2}} S^{1} \subset M_{n}$ e temos que $f$ se extende a uma aplicação $\tilde{f}: M_{n} \mapsto M_{n}$ de grau zero, com uma única classe de ponto fixo $C$, usando-se o número de Lefschetz obtemos que $|\operatorname{ind}(C)|=n$.

Observamos agora que:

$$
M_{n}=\left\{\begin{array}{cc}
K_{\frac{n-1}{2}} & \text { se } n \text { é ímpar } \\
P_{\frac{n}{2}} & \text { se } n \text { é par }
\end{array}\right.
$$

o que finaliza a demostração.

\footnotetext{
${ }^{15}$ Note que $\pi_{1}\left(M_{n}\right)$ tem $n+1$ geradores $\left\{\gamma_{1}, \gamma_{2}, \cdots, \gamma_{n+1}\right\}$ que satisfazem a relação $\gamma_{1}^{2} \gamma_{2}^{2} \cdots \gamma_{n+1}^{2}=1$

${ }^{16} \mathrm{O}$ "bouquet", denotado $\bigvee S^{1}$.
} 


\section{Referências Bibliográficas}

[Baues] Baues, Hans J. Obstruction Theory - On Homotopy Classification of Maps, Lectures Notes in Math. 628, Springer-Verlag; 1977.

[Brooks] Brooks, R.B. S. Coincidence, Roots and Fixed Points, Doctorial Dissertation, University of California, 1967.

[BBPT] Brooks, R.B. S.; Brown, R. F.; Pak, J.; Taylor, P. H. Nielsen numbers of maps of tori, Proc. Amer. Math. Soc. 52 (1975), 398-400.

[Dobreńko \& Jezierski] Dobreńko, R.; Jezierski, J. The coincidence Nielsen theory on non-orientable manifolds, Rocky Mountain J. math. 23 (1993), 67-85.

[Dold] Dold, A. Fixed Point Index and Fixed Point Theorem for Euclidean Neighborhood Retracts. Topology 4(1965), 1-8.

[Epstein] Epstein, D. B. A.; The Degree of a Map, Proc. London Math. Soc. (3), 16 (1966), 369-383.

[Gonçalves] Gonçalves, Daciberg L. Coincidence Theory for Maps from a Complex into a Manifold. Topology Appl. 92 (1999), 63-79.

[Gonçalves \& Jezierski] Gonçalves, Daciberg L.; Jezierski, J. Lefschetz coincidence formula on non-orientable manifolds, Fund. Math. 153 (1997), 01-23.

[Gonçalves \& Jiang] Gonçalves, Daciberg L.; Jiang, Boju. The index of coincidence Nielsen classes of maps between surfaces, Topology Appl. 116 (2001), 73-89.

[Gonçalves \& Kiihl] Gonçalves, Daciberg L.; Kiihl, José Carlos de S.Teoria do Índice, $14^{\circ}$ Colóquio Brasileiro de Matemática, Instituto de Matemática Pura e Aplicada do CNPq, 1983. 
[GKZ] Gonçalves, Daciberg L.; Kudryavtseva, Elena; Zieschang, H. Roots of mappings on nonorientable surfaces and equations in free groups, a aparecer em Manuscripta Mathematica.

[Gonçalves \& Oliveira] Gonçalves, Daciberg L.; Oliveira, Edson, The Lefschetz coincidence number for maps among compact surfaces, Far East J. Math. Sci., Special Vol., Part II (1997) 147-166.

[Hu] Hu, Sze-Tsen; Homology Theory: A First Course in Algebraic Topology, Holden-Day; 1966.

[Jezierski] Jezierski, J. The Nielsen coincidence theory on topological manifolds, Fund. Math. 143 (1993), 167-178.

[Jiang 1] Jiang, Boju. Lectures on Nielsen Fixed Point Theory. Contemporary Mathematics, vol 14; 1982.

[Jiang 2] Jiang, Boju; Bounds for fixed points on sufaces, Math. Ann. 311 (1998), $467-478$.

[Jiang \& Guo] Jiang, Boju; Guo, Jianhan. Fixed Points of Surface Diffeomorphisms, Pacific J. Math. 160 (1993), 67-89.

[Kelly 1] Kelly, Michael R. A bound on the fixed-point index for surface mappings. Ergod. Th. \& Dynam. Sys. 17 (1997), 1394-1408.

[Kelly 2] Kelly, Michael R. Bounds on the fixed point indices for self-maps of certain simplicial complexes. Topology Appl. 108 (2000), 179-196.

[Lyndon \& Schupp] Lyndon, Roger C.; Schupp, Paul E. Combinatorial Group Theory, Ergebnisse der Mathematik und ihrer Grenzgebiete 89, SpringerVerlag; 1977.

[MKS] Magnus, W.; Karrass, A.; Solitar, D. Combinatorial Group Theory, Interscience; 1966.

[Oliveira] Oliveira, Edson de. Teoria de Nielsen para Coincidência e Algumas Aplicações. Tese de Doutorado, USP, 1987.

[Olum 1] Olum, Paul. Obstructions to Extensions and Homotopies, Annals of Math. 52 (1950), 1-50.

[Olum 2] Olum, Paul. Mappings of Manifolds and the Notion of Degre, Annals of Math. 58 (1953), 458-480. 
[Pelikan \& Slaminka] Pelikan S.; Slaminka, E. E. A bound for the fixed point index of area-preserving homeomorphisms of two-manifolds, Ergod. Th. \& Dynam. Sys. 7 (1987), 463-479.

[Simon] Simon, S. A bound for the fixed point index of an area-preserving map with applications to mechanics. Inventiones Math. 26 (1974), 187-200

[Spanier] Spanier, E. Duality in topological manifolds, Colloque de Topologie Tenu à Bruxelles, Centre Belge de Recherches Mathématiques, 1966, 91-111.

[Steenrood 1] Steenrood, Norman Topology of Fibre Bundles, Princeton University Press; 1961.

[Steenrood 2] Steenrood, Norman Homology with Local Coefficients, Annals of Math. 44 (1943), 610-627.

[Stillwell] Stillwell, John. Geometry of Surfaces, Springer-Verlag; 1992.

[Vick] Vick, James W. Homology Theory An Introduction to Algebraic Topology, Academic Press; 1973.

[Whitehead] Whitehead, George W. Elements of Homotopy Theory, SpringerVerlag; 1978.

[ZVC] Zieschang, H.; Vogt, E.; Coldewey, H-D. Surface and Planar Discontinuos Groups, Lectures Notes in Math. 835, Springer-Verlag; 1980. 